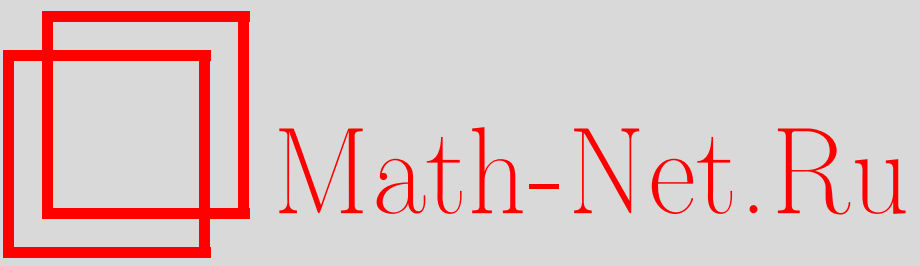

Э. Бак, Глобальные действия: алгебраический двойник топологического пространства, УМН, 1997, том 52, выпуск 5, 71-112

DOI: https://doi.org/10.4213/rm885

Использование Общероссийского математического портала Math-Net.Ru подразумевает, что вы прочитали и согласны с пользовательским соглашением

http://www.mathnet.ru/rus/agreement

Параметры загрузки:

IP: 54.162.27.143

26 апреля 2023 г., 13:09:41

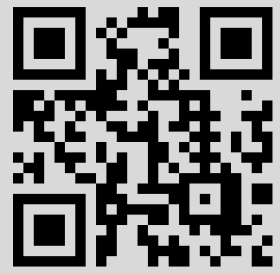




\title{
ГЛОБАЛЬНЫЕ ДЕЙСТВИЯ: АЛГЕБРАИЧЕСКИЙ ДВОЙНИК ТОПОЛОГИЧЕСКОГО ПРОСТРАНСТВА
}

\author{
Э. БАК
}

СОДЕРЖАНИЕ

1. Введение

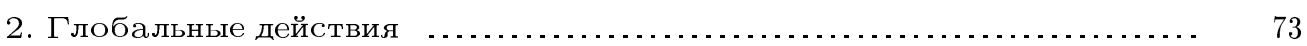

3. Морфизмы и пространства морфизмов ......................... 80

4. Относительные действия и их пространства морфизмов . ............ 93

\section{1. Введение}

В настояшей статье вводится общая алгебраическая концепия пространства с движением. Такое пространство состоит из множества $X$, рассматриваемого вместе с семейством групповых действий $G_{\alpha} \curvearrowright X_{\alpha}$, где $G_{\alpha}-$ группа, действуюшая на подмножестве из $X_{\alpha} \subseteq X$. Допускается, что на данном подмножестве из $X$ действует несколько различных групп. Групповые действия нумеруются элементами $\alpha$ из некоторого индексного множества $\Phi$, назьваемого координатной системой. Мы структурируем множество групповых действий $\left\{G_{\alpha} \curvearrowright X_{\alpha} \mid \alpha \in \Phi\right\}$, снабжая $\Phi$ транзитивным рефлексивным отношением $\leqslant$ и требуя, чтобы группы $G_{\alpha}$ определяли функтор $G$ : $\Phi \rightarrow\{$ групшы $\}, \alpha \mapsto G_{\alpha}$, удовлетворяющий следующему условию: если $\sigma \in G_{\alpha}$ и $\rho$ - его образ в $G_{\beta}$ при гомоморфизме $G_{\alpha \leqslant \beta}$, то для любого $x \in X_{\alpha} \cap X_{\beta}$ выполняется равенство $\sigma x=\rho x$. Результирующая тройка $(\Phi, G, X)$ назьвается глобальным действием. Движение вводится с помошњю понятия пути. Путь - это такая последовательность $x_{0}, \ldots, x_{p}$ точек из $X$, что для каждого $0 \leqslant i \leqslant p-1$ найдется координата $\alpha_{i} \in \Phi$ со следуюшим свойством: $x_{i}, x_{i+1} \in X_{\alpha_{i}}$ и $\sigma_{i} x_{i}=x_{i+1}$ для некоторого $\sigma_{i} \in G_{\alpha_{i}}$. Оказьвается, что сушествует такое глобальное действие $L$, называемое линейньм действием, что пути в данном глобальном действии $A$ определяются морфизмами из $L$ в $A$. Мы будем использовать эту естественную и интуитивно ясную конструкцию для того, чтобы перенести в алгебру весь опыт работы с путями в топологических пространствах. Одним из следствий этой программы будет гомотопическая теория для алгебраических структур, включающая в себя естественное, интуитивно ясное и, в то же время, строгое понятие алгебраической деформации морфизмов. Для реализации этой цели необходимо создать две важные конструкции, и обе они находят отражение в настояшей статье. Прежде всего, требуется показать, как превратить в глобальное действие множество $\operatorname{Mor}(A, B)$ всех морфизмов из глобального действия $A$ в глобальное действие $B$. Такая конструкция позволяет продеформировать мор- 
физм $f: A \rightarrow B$ в морфизм $g: A \rightarrow B$ с помощью пути из $f$ в $g$. Во вторых, необходимо сформулировать для глобальных действий общее условие, гарантирующее, что экспоненциальное отображение $E: \operatorname{Mor}(A, \operatorname{Mor}(B, C)) \rightarrow \operatorname{Mor}(A \times B, C)$ является изоморфизмом глобальных действий. В результате мы получаем, что цилиндрический метод для деформации морфизма $f$ в морфизм $g$ эквивалентен процедуре, используюшей упоминавшиеся выше пути. С помошью этой эквивалентности можно развить основные конструкции и принщипы алгебраической гомотопической теории, копируюшие их топологические аналоги. Это будет сделано в следуюшей статье.

Чтобы применить алгебраическую гомотопическую теорию к деформации морфизмов в произвольных категориях, требуется понятие глобального объекта. Это понятие также вводится в настоящей статье. Оно обобщает на произвольную категорию понятие глобального действия и позволяет раскрыть глубину и перспективы этого понятия.

За пределами чистой математики глобальные объекты и действия доставляют новую среду и язьк для моделирования проблем физики, химии, биологии и технических наук.

Статья организована следующим образом. Во втором параграффе вводится понятие глобального действия, которое иллюстрируется многочисленньми примерами. Среди них упоминавшееся вьше глобальное действие $L$, некоторые примеры, происходяшие из геометрии, а также глобальные действия, которые мы назвали моделями Володина. Модели Володина будут использоваться в следующей статье для алгебраического определения высших К-груш Володина и для разработки алгебраических основ алгебраической K-теории. Затем мы вводим понятие глобального объектаи представления глобальных объектов глобальньпи действиями. Приведены несколько примеров, показьваюших, что происходяшие из геометрии глобальные действия, описанные в начале параграфа, являются представлениями глобальных симплициальных комплексов, т.е. глобальных объектов в категории (абстрактных) симплициальных комплексов. Параграф завершается двумя результатами, которые описьвают функториальную конструкцию для построения глобальных объектов из примитивных данных. Эта конструкция окажется весьма полезной для разработки теории деформаций морфизмов в произвольных категориях.

В третьем параграфе изучается понятие морфизмаглобальных действий. Рассматривается обшее понятие морфизма, а также два его важных частных случая - нормальные и регулярные морфизмы. Регулярные морфизмы доставляют нам самое сильное понятие морфизма и сохраняют все структурные элементы в определении глобальных действий. Общее понятие морфизма учитьвает действия индивидуальных груп, но не отражает связей между действиями, задаваемых транзитивным рефлексивньм отношением на координатной системе $\Phi$ и групповым функтором $G$. Нормальные морфизмы лежат где-то между регулярньми и общими морфизмами. Все регулярные морфизмы нормальны; обратное, в общем случае, не верно.

Вначале мы определим общее понятие морфизма, а затем - регулярные морфизмы. Вводится понятие карты, которое используется для определения структуры глобального действия на множестве $\operatorname{Mor}(A, B)$ всех морфизмов из глобального действия $A$ в глобальное действие $B$. Как глобальное действие $\operatorname{Mor}(A, B)$ является контравариантным функтором по первому аргументу, однако он не определен для всех морфизмов по второму аргументу. Мы вводим понятие нормального морфизма так, что $\operatorname{Mor}(A, B)$ становится ковариантньм функтором по второму аргументу относительно этих морфизмов. Это обстоятельство будет очень полезно для алгебраической гомотопической теории, поскольку оно позволит доказать, что алгебраические гомотопические груп- 
пы функториальны относительно обширного класса нормальных морфизмов, назьваемых $\infty$ - $L$-морфизмами. Затем мы вводим глобальные действия с нижней гранью и сильной нижней гранью. Модели Володина, а также глобальные действия, происходяшие из геометрии, являются естественньми примерами глобальных действий с сильной нижней гранью. Показано, что любой морфизм, область значений которого - действие с нижней гранью или сильной нижней гранью, является $\infty$-нормальным и что экспоненциальньй морфизм $E: \operatorname{Mor}(A, \operatorname{Mor}(B, C)) \rightarrow \operatorname{Mor}(A \times B, C)$ является $\infty$-нормальньм изоморфизмом для действия $C$ с нижней гранью и регулярным изоморфизмом для действия $C$ с сильной нижней гранью. Эти результаты также потребуются нам для разработки алгебраической гомотопической теории.

В четвертом параграффе вводятся понятия поддействия и относительного действия. Относительное действие - это пара, состоящая из глобального действия и его поддействия. Относительные действия являются неотъемлемой составной частью гомотопической теории глобальных действий. В четвертом параграфе для относительных действий полностью повторяется вся программа, которая была разработана в предыдущем параграфе для глобальных действий. Детали здесь не столь рутинны, как в случае топологических пространств. Дополнительные сложности возникают в связи с понятием относительной карты, которая нужна для задания относительной глобальной структуры на множестве $\operatorname{Mor}(A, B)$ всех морфизмов из относительного действия $A$ в относительное действие $B$. Относительные карты - более сложньй объект, чем их абсолютный аналог, и эта дополнительная сложность проходит через весь параграф. Полученные в нем результаты полностью аналогичны результатам третьего параграфа.

Настоящая статья написана в элементарном стиле и является самозамкнутой. Для ее понимания требуются простейшие понятия, относяшиеся к множествам, группам, симплициальньм комплексам и категориям. Полезно знакомство с алгебраической топологией и алгебраической К-теорией.

Мне очень приятно выразить благодарность CONICET-DAAD и моему аргентинскому коллеге Гиермо Кортинасу за возможность прочесть курс по глобальньп действиям и неабелевой К-теории в университетах Буэнос Айреса и Ла Пиаты в 1995 г. Я особо признателен Элизабет Д'Альфонсо, которая записала мои лекции - эти записи были очень полезны при подготовке настояшей статьи. Кроме того, я обязан Алексею Степанову, чьи конспекты моего семинара по глобальным действиям также были для меня очень полезны.

\section{2. Глобальные действия}

Глобальное действие - это алгебраический объект, которьй собирается или склеивается из различных групповых действий. Глобальное действие напоминает те хорошо известные математические объекты, которые получаются путем сборки из простых строительных блоков и, в результате, образуют сложные структуры. Примерами таких объектов являются симплищиальные комплексы, строительньми блоками которых служат симплексы, клеточные комплексы, составленные из замкнутых дисков, многообразия, в которых строительные блоки - это открытые диски фиксированной размерности, и алгебраические многообразия (соответственно, схемы), где строительные блоки - это аффинные алгебраические многообразия (соответственно, аффинные схемы).

ОПРЕДЕЛЕНИЕ 2.1. Глобальным действием называется семейство $\left\{G_{\alpha} X_{\alpha} \mid \alpha \in \Phi\right\}$ групш $G_{\alpha}$, действующих на подмножествах $X_{\alpha}$ некоторого множества $|X|$ и удовлетво- 
ряющих следуюшим условиям.

(2.1.1) Множество индексов $\Phi$ снабжено транзитивньм и рефлексивньм отношением $\leqslant$. Если $\leqslant$ является отношением частичного порядка, т.е. если из условий $\alpha \leqslant \beta$ и $\beta \leqslant \alpha$ вытекает, что $\alpha=\beta$, то глобальное действие называется частично упорядоченнылм действием.

Множество $\Phi$ с отношением $\leqslant$ будет часто рассматриваться как категория. В таком случае для любых двух объектов $\alpha, \beta \in \Phi$ существует по крайней мере один морфизм $\alpha \leqslant \beta$. Если одновременно $\alpha \leqslant \beta$ и $\beta \leqslant \alpha$, то эти морфизмы считаются взаимно обратными.

(2.1.2) В обшем случае функция $X: \Phi \rightarrow\{$ подмножества из $|X|\}, \alpha \mapsto X_{\alpha}$, не имеет каких-либо специальных свойств. Если же отношение $\leqslant$ является отношением частичного порядка, а множество подмножеств из $|X|$ рассматривается как частично упорядоченное по включению, и если упомянутая функция $X$ обрашает порядок, то глобальное действие назьвается контраватиантным.

(2.1.3) Сопоставление $G: \Phi \rightarrow\{$ группы $\}, \alpha \mapsto G_{\alpha}$ является ковариантным функтоpom.

(2.1.4) Условие согласованности. Если $\alpha \leqslant \beta$, то группа $G_{\alpha}$ оставляет инвариантным множество $X_{\alpha} \cap X_{\beta}$ и для всех $\sigma \in G_{\alpha}$ и $x \in X_{\alpha} \cap X_{\beta}$ вьполняется соотношение $\sigma x=G_{\alpha \leqslant \beta}(\sigma) x$. (Отметим, что для пустого множества $X_{\alpha} \cap X_{\beta}$ условие согласованности вьполняется автоматически.)

Множество $\Phi$ называется координатной системой действия, а любой элемент из $\Phi$ назьвается координатой. Функтор $G$ называется глобальнылм групповылм функтором действия, функция $X$ назьвается глобальной функиией множсеств, $|X|$ называется обертывающим множеством. Если $\alpha$ - некоторая координата, то $G_{\alpha}$ называется локальной группй, а $X_{\alpha}$ - локальным множеством, отвечаюшим $\alpha$. Пусть $x \in|X|$. Будем говорить, что локальная группа $G_{\alpha}$ или же элемент $\sigma \in G_{\alpha}$ определены в точке $x$, если $x \in X_{\alpha}$. Групповым әлементом глобального действия назьваем элемент некоторой локальной групш $G_{\alpha}$.

ЗАмЕЧАнИЕ. Многие глобальные действия обладают дополнительным свойством: $|X|=\bigcup_{\alpha \in \Phi} X_{\alpha}$ или даже $|X|=X_{\alpha}$ для некоторого $\alpha \in \Phi$. Если же у глобального действия это свойство отсутствует, то его можно получить, добавив к $\Phi$ такой элемент $*$, что $*<\alpha$ для всех $\alpha \in \Phi$, и затем положив $X_{*}=|X|$ и $G_{*}=\{1\}$. Однако это добавление может несколько изменить структуру глобального действия - мы убедимся в этом на примере $\infty$-экспоненциального действия (3.18), а также при доказательстве теоремы 3.23 .

Разрешается, чтобы для некоторых координат $\alpha$ вьполнялось условие $X_{\alpha}=\varnothing$. Это удобно в некоторых конструкщиях, поскольку отпадает необходимость проверять всякий раз, является ли $X_{\alpha}$ непустым множеством.

Приведем теперь несколько примеров, иллюстрируюших понятие глобального действия.

ПримеР 2.2. Пусть $G$ - группа, действующая на множестве $|X|$, и $\Phi$ - множество, индексируюшее совокупность $\left\{G_{\alpha} \mid \alpha \in \Phi\right\}$ всех ее подгрупп. Будем считать, что $G_{\alpha}=G_{\beta} \Leftrightarrow \alpha=\beta$. Частично упорядочим множество $\left\{G_{\alpha} \mid \alpha \in \Phi\right\}$ по включению и зададим на $\Phi$ индуцированное отношение частичного порядка. Ясно, что соответствие $\alpha \mapsto G_{\alpha}$ задает функтор $\Phi \rightarrow\{$ групшы $\}$. Определим функцию $X: \Phi \rightarrow\{$ подмножества из $|X|\}$, полагая $X_{\alpha}=|X|$ для всех $\alpha \in \Phi$. В результате мы получим глобальное контравариантое действие $(\Phi, G, X)$. 
ОПРЕДЕЛЕНИЕ 2.3. Предположим, что в примере 2.2 в качестве множества $|X|$ взята сама группа $G$ и что $G$ действует на $|X|$ умножением. Кроме того, предположим, что $G_{*}=\{1\}$ для некоторого $* \in \Phi$, множество $\left\{G_{\alpha} \mid \alpha \in \Phi\right\}$ замкнуто относительно пересечений и что выполняется следуюшее условие: если $G_{\alpha}$ и $G_{\beta}$ содержатся в некоторой подгрупе $G_{\gamma}$, то подгруппа $\left(G_{\alpha}, G_{\beta}\right) \subset G$, порожденная $G_{\alpha}$ и $G_{\beta}$ совпадает с подгруппой $G_{\gamma}$. Тогда глобальное действие $(\Phi, G, X)$ называется моделью Володина. (Это название объясняется тем, что К-групшы Володина для колец или для колец с инволюцией представляют собой алгебраические гомотопические групы некоторых моделей Володина, см. $\S 6$. Замкнутость множества $\left\{G_{\alpha} \mid \alpha \in \Phi\right\}$ относительно пересечений используется при доказательстве совпадения алгебраических гомотопических групп модели Володина с обычншми гомотопическими групшами.)

Пусть $U-$ некоторое множество. Положим

$\operatorname{Perm}(U)=\operatorname{Aut}_{\text {sets }}(U)$

$f \operatorname{Perm}(U)=\{\sigma \in \operatorname{Perm}(U) \mid \sigma$ оставляет неподвижными почти все элементы из $U\}$.

Если $U$ - линейно упорядоченное множество, обозначим через $c \operatorname{Perm}(U)$ циклическую подгруппу из $\operatorname{Perm}(U)$, порожденную перестановкой, которая переводит каждьй элемент из $U$, за исключением последнего, в следуюший за ним элемент, а последний элемент - в первьй.

ПримеР 2.4. В этом примере мы рассмотрим так назьваемое линейное действие; оно будет играть важную роль в гомотопической теории глобальных действий. Пусть $\Phi=\mathbb{Z} \cup\{*\}$. Зададим на $\Phi$ структуру частично упорядоченного множества, считая элементы из $\mathbb{Z}$ несравнимыми и $* \leqslant n$ для всех $n \in \mathbb{Z}$. Положим $|X|=\mathbb{Z}$ и зададим функцию $X: \Phi \rightarrow\{$ подмножества $|X|\}$, правилом $\alpha=n \mapsto\{n, n+1\}, \alpha=* \mapsto|X|$, a функтор $G: \Phi \mapsto\{$ группы $\}$ - правилом $\alpha=n \mapsto G_{\alpha}=\operatorname{Perm}(\{n, n+1\}), \alpha=$ $* \mapsto G_{\alpha}=\{1\}$. Тогда тройка $(\Phi, G, X)$ является контравариантным действием.

Рассмотренньй пример допускает следуюшее обобщение.

ПримеР 2.5. Пусть $S$ - абстрактньй симплициальньй комплекс и $|X|-$ множество вершин $S$. Если $\alpha$ - подкомплекс из $S$, обозначим через $X_{\alpha}$ множество его вершин. Назовем подкомплекс $\alpha$ простым, если $X_{\alpha}$ допускает разбиение на такие подмножества из $U$, что каждому конечному подмножеству из $U$ отвечает симплекс и что вершины любого симплекса из $\alpha$ лежат в некотором $U$. Ясно, что если $\alpha-$ простой подкомплекс, то такое разбиение единственно; мы обозначим его через $\operatorname{Part}\left(X_{\alpha}\right)$. Определим теперь $\Phi$ как множество всех простых подкомплексов из $S$. Частично упорядочим $\Phi$, полагая $\alpha \leqslant \beta$ тогда и только тогда, когда $X_{\alpha} \supseteq X_{\beta}$ и каждый элемент из $\operatorname{Part}\left(X_{\beta}\right)$ является объединением элементов из $\operatorname{Part}\left(X_{\alpha}\right)$. Очевидно, что подкомплекс с множеством вершин $|X|$ представляет собой наименьший элемент в $\Phi$. Для $\alpha \in \Phi$ положим

$$
\begin{aligned}
G_{\alpha} & =\prod_{U \in \operatorname{Part}\left(X_{\alpha}\right)} \operatorname{Perm}(U), \\
f G_{\alpha} & =\prod_{U \in \operatorname{Part}\left(X_{\alpha}\right)} f \operatorname{Perm}(U) .
\end{aligned}
$$

На множествах $X_{\alpha}$ существует каноническое действие груп $G_{\alpha}$ (соответственно $\left.f G_{\alpha}\right)$, определяемое действием каждой из груп перестановок $\operatorname{Perm}(U)$ (соответственно $f \operatorname{Perm}(U))$ на своем множестве $U$. Положим по определению

$$
\begin{aligned}
\operatorname{gl}(S) & =(\Phi, G, X), \\
f \operatorname{gl}(S) & =(\Phi, f G, X) .
\end{aligned}
$$


Тогда $\operatorname{gl}(S)$ и $f \operatorname{gl}(S)$ являются глобальными действиями; мы назовем их симллиииальньлми действиями.

Линейно упорядочим вершины $|X|$ симплициального комплекса $S$ и обозначим через $c \Phi$ подмножество в $\Phi$, состояшее из тех простых подкомплексов $\alpha$, у которых $\operatorname{Part}\left(X_{\alpha}\right)$ содержит только конечные множества. Ясно, что наименьший элемент $*$ из $\Phi$ лежит в $c \Phi$. Зададим на $c \Phi$ новое частичное упорядочение, полагая $\alpha \leqslant \beta \Leftrightarrow \alpha=*$. Таким образом, если $\alpha \neq * \neq \beta$, то или $\alpha=\beta$, или же $\alpha$ и $\beta$ несравнимы. Пусть $\alpha \in c \Phi$. Положим

$$
\begin{aligned}
(c X)_{\alpha} & =X_{\alpha}, \\
c G_{\alpha} & =\prod_{U \in \operatorname{Part}\left(X_{\alpha}\right)} c \operatorname{Perm}(U), \\
c \operatorname{gl}(S) & =(c \Phi, c G, c X) .
\end{aligned}
$$

Тройка $c \operatorname{gl}(S)$ назьвается циклическим симплициальным действием.

Чтобы подготовиться к более сложным примерам, напомним некоторые понятия из теории категорий.

Пусть $\mathscr{C}$ - некоторая категория, $O$ - объект из $\mathscr{C}$ и $O^{\prime} \longmapsto O$ - подобъект объекта $O$. Говорят, что автоморфизм $\sigma \in \operatorname{Aut}_{\mathscr{C}}(O)$ оставляет $O^{\prime}$ инвариантным, если найдется такой автоморфизм $\rho \in \operatorname{Aut}_{\mathscr{C}}\left(O^{\prime}\right)$, что имеет место коммутативная диаграмма

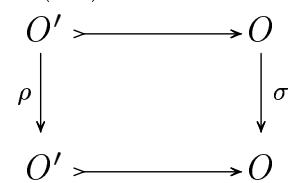

Ясно, что если автоморфизм $\rho$ существует, то он определяется единственньгм образом. Множество всех автоморфизмов объекта $O$, оставляющих инвариантным подобъект $O^{\prime}$, образует подгрупу

$$
\operatorname{Stab}_{O}\left(O^{\prime}\right)
$$

в $\operatorname{Aut}_{\mathscr{C}}(O)$, назьваемую стабилизатором $O^{\prime}$ в $O$. Сушествует канонический гомоморфизм

$$
\begin{aligned}
\operatorname{Stab}_{O}\left(O^{\prime}\right) & \rightarrow \operatorname{Aut}_{\mathscr{C}}\left(O^{\prime}\right), \\
\sigma & \mapsto \rho .
\end{aligned}
$$

Пусть $P$ - некоторьй объект из $\mathscr{C}$. Назовем $P$-точкой в $\mathscr{C}$ элемент $\operatorname{Mor}_{\mathscr{C}}(P, O)$, где $O$ - произвольньй объект. Для фиксированного $P$ понятие $P$-точки позволяет связать с любым объектом $O$ из $\mathscr{C}$ подстилающее множество $\operatorname{Mor}_{\mathscr{C}}(P, O)$ всех его $P$-точек. Более того, для любого множества $\mathscr{P}$ объектов из $\mathscr{C}$ (в предположении, что никакие два различных объекта из $\mathscr{P}$ не изоморфны) можно определить $n о д c m u-$ лающее множсество $\mathscr{P}$-точек объекта $O$ как $\bigcup_{P \in \mathscr{P}} \operatorname{Mor}_{\mathscr{C}}(P, O)$. Если $O^{\prime} \longmapsto O-$ подобъект, то существует каноническое вложение множеств

$$
\mathscr{P} \text {-точки }\left(O^{\prime}\right) \subseteq \mathscr{P} \text {-точки }(O) \text {. }
$$

Обобщим теперь понятие глобального действия, определив так назьваемьй глобальньй объект, и разовьем метод для построения глобальных объектов из простых данных. Это позволит нам получить много содержательных примеров глобальных объектов и глобальных действий и даст возможность применить методы глобальных действий к различным задачам. 
ОПРеДЕЛЕНИЕ 2.6. Пусть $\mathscr{C}$ - некоторая категория. Глобальный оббект в $\mathscr{C}$ состоит из множества $\left\{O_{\alpha} \longmapsto O \mid \alpha \in \Phi\right\}$ подобъектов $O_{\alpha} \longmapsto O$ объекта $O$ и множества $\left\{G_{\alpha} \rightarrow \operatorname{Aut}_{\mathscr{C}}\left(O_{\alpha}\right) \mid \alpha \in \Phi\right\}$ групп $G_{\alpha}$ и гомоморфизмов $G_{\alpha} \rightarrow \operatorname{Aut}_{\mathscr{C}}\left(O_{\alpha}\right)$, удовлетворяюших следуюшим условиям.

(2.6.1) Множество $\Phi$ снабжено транзитивньм рефлексивных отношением $\leqslant$. Если $\leqslant$ является отношением частичного порядка, то глобальньй объект называется частично упорядоченныцм облектом.

(2.6.2) Функция $O: \Phi \longrightarrow$ пподобъекты $(O)\}, \alpha \mapsto\left(O_{\alpha} \longmapsto O\right)$, в общем случае не имеет каких-либо специальных свойств. Если же отношение $\leqslant$ является отношением частичного порядка, а на множестве подобъектов в $O$ задано каноническое транзитивное реф лексивное отношение, индуцированное включением подобъектов, и если функция $O$ обрашает отношения, то глобальньй объект называется контравариантным.

(2.6.3) Сопоставление $G: \Phi \longrightarrow\{$ групшы $\}, \alpha \mapsto G_{\alpha}$, является ковариантным функтором.

(2.6.4) Условие согласованности. Если $\alpha \leqslant \beta$, то в категории $\mathscr{C}$ существуют расслоенное произведение

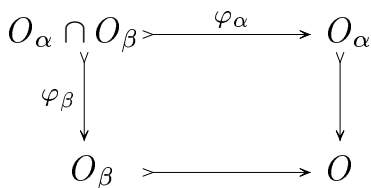

и такой (однозначно определенньй) гомоморфизм $G_{\alpha} \rightarrow \operatorname{Aut} \mathscr{C}\left(O_{\alpha} \cap O_{\beta}\right)$, что морфизмы $\varphi_{\alpha}$ и $\varphi_{\beta} G_{\alpha}$-эквивариантны.

С точки зрения этого определения глобальное действие есть не что иное, как глобальное множество (глобальный объект в категории множеств).

ОПРЕДЕЛЕНИЕ 2.7 . Пусть $(\Phi, G, O)$ - глобальный объект в кагории $\mathscr{C}$ и $P$ - множество объектов из $\mathscr{C}$. Назовем $P$-представлением глобального объекта $(\Phi, G, O)$ множество $\left\{X_{\alpha} \mid \alpha \in \Phi, X_{\alpha} \subseteq P\right.$-точки $\left(O_{\alpha}\right), X_{\alpha}$ является $G_{\alpha}$-инвариантным $\}$. Каноническое $P$-представление глобального объекта $(\Phi, G, O)$ - это множество $\left\{P\right.$-точки $\left.O_{\alpha} \mid \alpha \in \Phi\right\}$. Легко проверить, что тройка $(\Phi, G, X)$ является глобальньм действием. Каноническое $P$-представление контравариантного объекта является контравариантным действием, для произвольного же $P$-представления это свойство, вообще говоря, не вьполняется.

Пример 2.8. Пусть $\mathscr{C}$ - категория абстрактных симплициальных комплексов, $S$ - некоторьй объект из $\mathscr{C}$. Определим множество $\Phi$ так же, как в примере 2.5 и для каждого $\alpha \in \Phi$ положим $S_{\alpha}=\alpha$. Таким образом, $S_{\alpha}-$ это простой подкомплекс комплекса $S$. Пусть $G_{\alpha}$ и $f G_{\alpha}$ определены так же, как в 2.5. Тогда $G_{\alpha}$ и $f G_{\alpha}$ являются подгрупшами групп автоморфизмов $\operatorname{Aut}_{\mathscr{C}}\left(S_{\alpha}\right)$. Без труда проверяется, что $(\Phi, G, S)$ и $(\Phi, f G, S)$ - глобальные симплициальные комплексы. Кроме того, если $P$ - симплициальньй комплекс ровно с одной вершиной, то каноническое представление объекта $(\Phi, G, S)$ (соответственно $(\Phi, f G, S)$ ) есть не что иное, как глобальное действие $\mathrm{gl}(S)$ (соответственно $f \mathrm{gl}(S)$ ), определенное в 2.5 .

Пусть множество $c \Phi$ определено так же, как в примере 2.5 . Для каждого $\alpha \in c \Phi$ положим $(c S)_{\alpha}=\alpha$. Тогда тройка $(c \Phi, c G, c S)$ является глобальным симплициальным комплексом, а его каноническое $P$-представление есть глобальное действие $c \operatorname{gl}(S)$, определенное в 2.5 .

Следующий метод построения глобальных объектов из локальных данных обобщает пример 2.2 и оказывается весьма полезным в приложениях. 
КонСтРУКЦИя-ЛЕМма 2.9. Пусть $\mathscr{C}$ - некоторая категория и $O$ - облект из $\mathscr{C}$.

(2.9.1) Глобальные данные для $O$ состоят из множсества подоблектов $\left\{O_{\alpha} \longmapsto O \mid \alpha \in \Phi\right\}$ и множества nодәрупп $\left\{G_{\alpha} \subseteq \operatorname{Aut}_{\mathscr{C}}\left(O_{\alpha}\right) \mid \alpha \in \Phi\right\}$.

(2.9.2) Для фиксированных глобальных данных определим транзитивное рефлексивное отношение $\leqslant_{c c}$ на $\Phi$, называемое каноническим контравариантным отношением. Будем считать, что $\alpha \leqslant c c \beta$ тогда и только тогда, когда существует такая коммутативная диаграмма

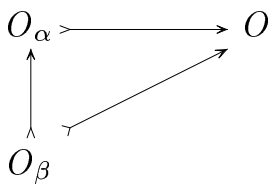

что $G_{\alpha} \subseteq \operatorname{Stab}_{O_{\alpha}}\left(O_{\beta}\right)$ и образ канонического гомоморфизма $G_{\alpha} \rightarrow \operatorname{Aut}_{\mathscr{C}}\left(O_{\beta}\right)$ лежит в $G_{\beta}$. Прямая проверка показывает, что тройка $(\Phi, G, O)$ является глобальным облектом с контравариантным функтором $O: \Phi \rightarrow\{$ подобгекты в $O\}$. Кроме того, если $\leqslant$ - такое транзитивное отношение на $\Phi$, ито $((\Phi, \leqslant), G, O)$ глобальный облект с контравариантным функтором $O: \Phi \longrightarrow\{$ подобвекты в $О\}$, то тождественное отобрахсение $\Phi \rightarrow \Phi$ является морфизмом $(\Phi, \leqslant) \rightarrow(\Phi, \leqslant c c)$ частично упорядоченных множеств.

(2.9.3) Оставляя без изменения глобальные данные, определим на $\Phi$ рефлексивное отношение $\leqslant_{c r}$, называемое каноническим отношением. Положим по определению, что $\alpha \leqslant_{c r} \beta$ тогда и только тогда, когда в категории $\mathscr{C}$ найдется такое расслоенное произведение

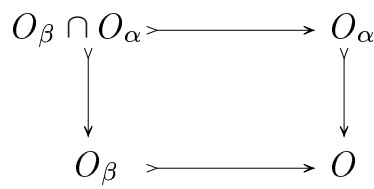

что группь $G_{\alpha}$ и $G_{\beta}$ содержатся в $\operatorname{Stab}_{O_{\alpha}}\left(O_{\beta} \cap O_{\alpha}\right)$, канонический гомоморфизм $G_{\beta} \longrightarrow \operatorname{Aut}_{\mathscr{C}}\left(O_{\beta} \cap O_{\alpha}\right)$ инбективен и образ $\left(G_{\alpha} \longrightarrow \operatorname{Aut}_{\mathscr{C}}\left(O_{\beta} \cap O_{\alpha}\right)\right)$ содерәится в образе предьдущего гомоморфизма. Следовательно, если $\alpha \leqslant c r ~ \beta$, то существует такой единственный гомоморфизм $G_{\alpha} \longrightarrow G_{\beta}$, что морфизм $O_{\beta} \cap O_{\alpha} \longrightarrow O_{\beta}$ является $G_{\alpha}$-эквивариантным. Пусть $\leqslant$ - некоторое транзитивное рефлексивное подотношение отношения $\leqslant_{c r}$, например, определенное выше отношение $\leqslant c c$. Предположим, что $\Phi$ наделено отношением $\leqslant$. Прямая проверка показывает, что тройка $(\Phi, G, O)$ - глобальный облект. Более того, если $\leqslant-$ произвольное транзитивное рефлексивное отношение на $\Phi$, такое что $((\Phi, \leqslant), G, O)$ - глобальный облект, то тожсдественное отображение $\Phi \rightarrow \Phi$ является морфизмом отношений $(\Phi, \leqslant) \longrightarrow(\Phi, \leqslant c r) . \quad$ В частности таким морфизмом является $\left(\Phi, \leqslant_{c c}\right) \longrightarrow(\Phi, \leqslant c r)$.

ДокАЗАТЕЛЬСТво. Единственными утверждениями, которые нужно доказать, являются утверждения, касаюшиеся отношений $\leqslant c c$ из (2.9.2) и $\leqslant c r$ из (2.9.3). Поскольку доказательства в том и другом случае аналогичны, мы ограничимся лишь отношением $\leqslant c c$. Предположим, что $\alpha \leqslant \beta$. Из контравариантности следует существование коммутативной диаграммы 


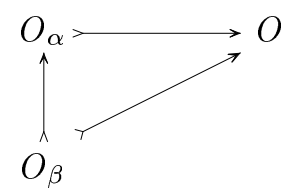

В силу условия согласованности (2.6.4) морфизм $O_{\beta} \longmapsto O_{\alpha}$ является $G_{\alpha}$-эквивариантным. Но это означает, что группа $G_{\alpha}$ оставляет инвариантньм подобъект $O_{\beta}$. Далее, ясно, что образ гомоморфизма $G_{\alpha} \rightarrow \operatorname{Aut}_{\mathscr{C}}\left(O_{\alpha}\right)$ должен лежать в $G_{\beta}$, поскольку сушествует ровно один гомоморфизм $G_{\alpha} \rightarrow \operatorname{Aut}_{\mathscr{C}}\left(O_{\alpha}\right)$, для которого $O_{\beta} \longmapsto O_{\alpha}$ является $G_{\alpha}$-эквивариантным морфизмом. Следовательно, $\alpha \leqslant c c \beta$, что и требовалось доказать.

Пусть $\Psi$ - некоторое множество индексов, $O$ - объект категории $\mathscr{C}$ и $O: \Psi \rightarrow$ $\{$ подобъектыв $(O)\}, \alpha \mapsto\left(O_{\alpha} \longmapsto O\right)$ - соответствуюшая функция. Обозначим через $\operatorname{Sub}_{\Psi}(O)$ категорию, объектами которой являются семейства $\left\{O_{\alpha} \mid \alpha \in \Psi\right\}$, а морфизмами - те единственные морфизмы подобъектов $O_{\alpha} \longmapsto O_{\beta}$, для которых коммутативна диаграмма

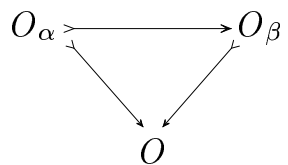

Если $S \subseteq \operatorname{Sub}_{\Psi}(O)$ - некоторая подкатегория и если в $\mathscr{S}$ существует копредел $\operatorname{colim} S$, то имеется канонический морфизм $\operatorname{colim} S \rightarrow O$.

Следующий метод построения глобальных объектов является обобщением метода, изложенного в примере 2.8 .

КонстрУкция-лЕмма 2.10. Пусть $\mathscr{C}$ - некоторая категория и $O-$ ее обтект.

(2.10.1) Рассмотрим множество $\left\{O_{\alpha} \longmapsto O \mid \alpha \in \Phi\right\}$ всех подобгектов $O_{\alpha} \longmapsto O$ оббекта О. Для каждого $\alpha \in \Phi$ обозначим через $\left\{O_{\alpha, i} \longmapsto O_{\alpha} \mid(\alpha, i) \in \Phi_{\alpha}\right\}$ множество подобғектов $O_{\alpha, i} \longmapsto O_{\alpha}$ обгекта $O_{\alpha}$, обладающих следующим свойством: в $\operatorname{Sub}_{\Phi_{\alpha}}\left(O_{\alpha}\right)$ найдется подкатегория $S_{\alpha} \subseteq \operatorname{Sub}_{\Phi_{\alpha}}\left(O_{\alpha}\right)$, для которой в $\mathscr{S}$ существует копредел $\operatorname{colim}\left(S_{\alpha}\right)$, причем канонический морфизм $\operatorname{colim}\left(S_{\alpha}\right) \rightarrow O_{\alpha}$ является изоморфизмом. Положим $G_{\alpha}=\left\{\sigma \in \operatorname{Aut}_{\mathscr{C}}\left(O_{\alpha}\right) \mid \sigma \in \operatorname{Stab}_{O_{\alpha}}\left(O_{\alpha, i}\right)\right.$ $\left.\forall(\alpha, i) \in \Phi_{\alpha}\right\}$. Тогда множества $\left\{O_{\alpha} \mapsto O \mid \alpha \in \Phi\right\}$ u $\left\{G_{\alpha} \subseteq \operatorname{Aut}_{\mathscr{C}}\left(O_{\alpha}\right) \mid \alpha \in \Phi\right\}$ определяют глобальные даннье в смысле (2.9.1).

(2.10.2) Отправляясь от этих глобальных данныц, определим на $\Phi$ следующее транзитивное рефлексивное отношение $\leqslant c c$. Будем считать, что $\alpha \leqslant c c \beta$ в том и только в том случае, когда найдется такая коммутативная диаграмма

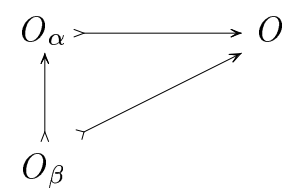

что для каждого $(\beta, j) \in \Phi_{\beta}$ облект $O_{\beta, j}$ является копределом семейства подобтектов $O_{\alpha, i} \longmapsto O_{\beta, j}$, входящих в следующий коммутативный квадрат:

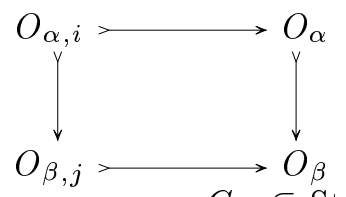

Из этого определения вытекает, что $G_{\alpha} \subseteq \operatorname{Stab}_{O_{\alpha}}\left(O_{\beta}\right)$ и образ канонического гомоморфизма $G_{\alpha} \rightarrow \operatorname{Aut}_{\mathscr{C}}\left(O_{\beta}\right)$ содержится в $G_{\beta}$. Применяя (2.9.2), мы 
получаем, что тройка $(\Phi, G, O)$ является глобальным обвектом с контравариантнылм $O$.

(2.10.3) Используя те же самые глобальные данные, что и выше, введем на $\Phi$ рефлексивное отношение $\leqslant_{c r}$. По определению $\alpha \leqslant_{c r} \beta$ тогда и только тогда, когда в $\mathscr{C}$ найдется такое расслоенное произведение

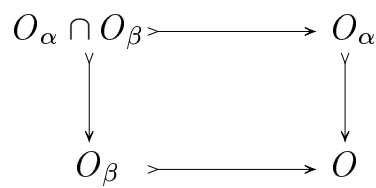

что $O_{\beta} \cap O_{\alpha}$ является копределом семейства подоблектов $O_{\alpha, i} \longmapsto O_{\alpha} \cap O_{\beta}$ (соответственно $\left.O_{\beta, j} \longmapsto O_{\alpha} \cap O_{\beta}\right)$, для которого имеет место коммутативная диаграмма

соответственно
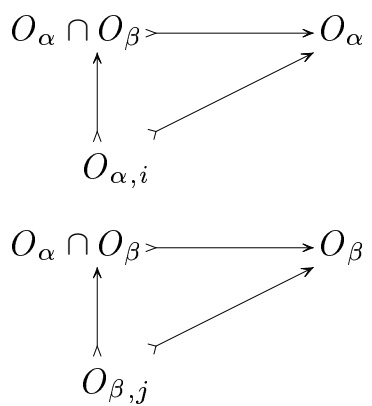

канонический гомоморфизм $G_{\beta} \rightarrow \operatorname{Aut}_{\mathscr{C}}\left(O_{\alpha} \cap O_{\beta}\right)$ инбективен и образ $\left(G_{\alpha} \rightarrow\right.$ $\left.\operatorname{Aut}_{\mathscr{C}}\left(O_{\alpha} \cap O_{\beta}\right)\right)$ содержится в образе предыдущего гомоморфизма. Пусть $\leqslant-н е-$ которое транзитивное рефлексивное подотношение отношения $\leqslant_{c r}$, например, определенное выше отношение $\leqslant c c$. Снабдим $\Phi$ әтим отношением $\leqslant$. Применяя (2.9.3), мы получаем, что тройка $(\Phi, G, O)$ является глобальным обтектом. Кроме того, тожддественне отображение $\Phi \rightarrow \Phi$ индуцирует морфизм отношений $(\Phi, \leqslant) \rightarrow(\Phi, \leqslant c r)$.

\section{3. Морфизмы и пространства морфизмов}

В этом параграфе мы определим понятие морфизма глобальных действий и выделим два важных специальных класса морфизмов - так называемые нормальные и регулярные морфизмы. На множестве $\operatorname{Mor}(A, B)$ всех морфизмов из глобального действия $A$ в глобальное действие $B$ мы зададим структуру глобального действия, преврашающую $\operatorname{Mor}($, , ) в контравариантньй функтор по первому аргументу относительно всех морфизмов и в ковариантный функтор по второму аргументу относительно нормальных морфизмов.

Регулярные морфизмы - самые "жесткие" из всех морфизмов и сохраняют все существенные структурные элементы в определении глобального действия. Два регулярно изоморфных гловальных действия по-существу идентичны. С другой стороны, изоморфные регулярные действия могут резко различаться, так как их структуры не обязаны быть взаимно однозначными. Например, такие действия могут иметь различные высшие алгебраические гомотопические группы, поскольку конструкция таких групп является функториальной только относительно некоторого класса морфизмов, содержашего регулярные морфизмы. Этот класс называется классом $\infty$ - $L$-нормальных морфизмов и будет также определен ниже.

Понятие морфизма зависит от понятий пути, локального пути и локального репера. 
Пусть $A$ - глобальное действие. Обозначим через $\Phi_{A}$ его координатную систему, $G_{A}$ - глобальньй групповой функтор, $X_{A}$ - глобальную функцию множеств и через $|A|$ - обертьвающее множество.

ОПРЕДЕлЕНИЕ 3.1. Пусть $A$ - глобальное действие.

(3.1.1) Путем в $A$ назьвается такая последовательность точек $x_{0}, \ldots, x_{p}$ из $|A|$, что для каждого $i(0 \leqslant i \leqslant p-1)$ существует групповой элемент $g_{i}$, определенный в $x_{i}$ и удовлетворяющий соотношению $g_{i} x_{i}=x_{i+1}$. Если $0=p$, то подразумевается, что $x_{0}$ лежит в некотором локальном множестве $\left(X_{A}\right)_{\alpha}$.

(3.1.2) Локальным путем в $\alpha \in \Phi_{A}$ назьвается такой путь $x_{0}, \ldots, x_{p}$ в $A$, что все $x_{i} \in\left(X_{A}\right)_{\alpha}$ и все $g_{i} \in\left(G_{A}\right)_{\alpha}$. (Очевидно, что если $x_{0}, \ldots, x_{p}$ - локальньй путь и $\pi-$ произвольная подстановка из $(p+1)$ элемента, то $x_{\pi\left(x_{0}\right)}, \ldots, x_{\pi(p)}$ - также локальньй путь.)

(3.1.3) Пусть $x \in\left(X_{A}\right)_{\alpha}$. Локальным репером в точке $x$ для индекса $\alpha$ или, короче, $\alpha$-репером в $x$ назьвается такая последовательность точек $x=x_{0}, \ldots, x_{p}$ из $\left(X_{A}\right)_{\alpha}$, что для каждого $i(1 \leqslant i \leqslant p)$ сушествует элемент $g_{i} \in\left(G_{A}\right)_{\alpha}$, для которого $g_{i} x=x_{i}$. (Очевидно, что $x, x_{1}, \ldots, x_{p}$ является $\alpha$-репером в $x$ тогда и только тогда, когда $x, x_{1}, \ldots, x_{p}$ - локальньй путь в $\alpha$.)

ОПРЕДЕЛЕНИЕ 3.2. Морфизмом $f: A \rightarrow B$ глобальных действий назьвается функция $f:|A| \rightarrow|B|$, которая сохраняет локальные реперы или, что эквивалентно, локальные пути. Это означает, что если $x_{0}, \ldots, x_{p}$ - некоторый $\alpha$-репер в точке $x_{0}$, то $f\left(x_{0}\right), \ldots, f\left(x_{p}\right)$ является $\beta$-репером для некоторого $\beta \in \Phi_{B}$.

ОПРеДЕЛЕНИЕ 3.3. Регулярным морфизмом $\eta: A \rightarrow B$ глобальных действий называется тройка $\left(\eta_{\Phi}, \eta_{G}, \eta_{X}\right)$, удовлетворяюшая следуюшим условиям.

(3.3.1) $\eta_{\Phi}: \Phi_{A} \rightarrow \Phi_{B}-$ функция, сохраняюшая отношения, т.е. ковариантньй функтор.

(3.3.2) $\eta_{G}: G_{A} \rightarrow\left(G_{B}\right)_{\eta_{\Phi}()}$ - естественное преобразование функтором на $\Phi_{A}$, где $\left(G_{B}\right)_{\eta_{\Phi}()}$ означает композищию $\eta_{\Phi}$ и $G_{B}$.

(3.3.3) $\eta_{X}:|A| \rightarrow|B|$ - такая функция, что $\eta_{X}\left(\left(X_{A}\right)_{\alpha}\right) \subseteq\left(X_{B}\right)_{\eta_{\Phi}(\alpha)}$ для всех $\alpha \in \Phi_{A}$.

(3.3.4) Для каждого $\alpha \in \Phi_{A}$, пара $\left(\eta_{G}, \eta_{X}\right):\left(G_{A}\right)_{\alpha} \curvearrowright\left(X_{A}\right)_{\alpha} \rightarrow\left(G_{B}\right)_{\eta_{\Phi}(\alpha)} \curvearrowright$ $\left(X_{B}\right)_{\eta_{\Phi}(\alpha)}$ является морфизмом групповых действий, т.е. для всех $\sigma \in\left(G_{A}\right)_{\alpha}$ и $x \in$ $\left(X_{A}\right)_{\alpha}$ вьполняется соотношение $\eta_{X}(\alpha)(g x)=\eta_{G}(\alpha)(\sigma)\left(\eta_{X}(\alpha)(x)\right)$. (Отсюда следует, в частности, что регулярный морфизм является морфизмом глобальных действий в смысле определения 3.2).

Регулярным изоморфизмом $\eta: A \rightarrow B$ назьвается регулярньй морфизм, для которого существует регулярньй морфизм $\eta^{\prime}: B \rightarrow A$ (назьваемьй регулярным обратнылм для $\eta$ ) со следующими свойствами: $\eta_{\Phi}^{\prime}$ является обратным для $\eta_{\Phi}, \eta_{X}^{\prime}$ - обратным для $\eta_{X}$, и для каждого $\alpha \in \Phi_{A}$ композиция $\eta_{G}^{\prime}\left(\eta_{\Phi}(\alpha)\right)$ является обратной для $\eta_{G}(\alpha)$.

Разумеется, регулярньй морфизм, являюшийся изоморфизмом, в обшем случае не будет регулярным изоморфиизмом.

Для того чтобы задать на множестве $\operatorname{Mor}(A, B)$ всех морфизмов из глобального действия $A$ в глобальное действие $B$ структуру глобального действия, нам понадобится понятие карты.

ОПРЕДЕЛЕНИЕ 3.4. Пусть $A$ и $B$ - глобальные действия. Назовем $A$-картой в $B$ пару $(f, \beta)$, состояшую из морфизма глобальных действий $f: A \rightarrow B$ и функции $\beta:|A| \rightarrow \Phi_{B}$, подчиненных следующим условиям.

(3.4.1) $f(x) \in\left(X_{B}\right)_{\beta(x)}$ для $x \in|A|$. 
(3.4.2) Если $x, x_{1}, \ldots, x_{p}$ - некоторьй $\alpha$-репер в точке $x \in|A|$, то $f(x), f\left(x_{1}\right), \ldots$, $f\left(x_{p}\right)$ является $b$-репером в точке $f(x)$ для некоторого $b \geqslant \beta(x), \beta\left(x_{1}\right), \ldots, \beta\left(x_{p}\right)$.

ОПРЕДЕЛЕНИЕ-ЛЕМма 3.5. Пусть $(f, \beta)$ - некоторая $A$-карта в $B$. Для

$$
\sigma=\left(\sigma_{x}\right) \in \prod_{x \in|A|}\left(G_{B}\right)_{\beta(x)}
$$

полоэм $и \mathcal{M}$

$$
\sigma f:|A| \rightarrow|B|, \quad x \mapsto \sigma_{x} f(x) .
$$

Тогда $\sigma f$ есть морфизм $A \rightarrow B$ глобальных действий, а $(\sigma f, \beta)$ - некоторая $A$-карта в $B$.

ДокАЗАТЕльство. Так как $\sigma_{x} \in\left(G_{B}\right)_{\beta(x)}$, то композиция $\sigma f(x) \in\left(X_{B}\right)_{\beta(x)}$. Следовательно, пара $(\sigma f, \beta)$ удовлетворяет условию (3.4.1). Поэтому, чтобы доказать лемму, достаточно проверить вьполнение условия (3.4.2). Пусть $x_{0}, \ldots, x_{p}$-локальньй репер в точке $x_{0} \in|A|$. По определению, $f\left(x_{0}\right), \ldots, f\left(x_{p}\right)$ является $b$-репером в точке $f\left(x_{0}\right)$ для некоторого $b \geqslant \beta\left(x_{0}\right), \ldots, \beta\left(x_{p}\right)$. Обозначим через $\rho_{x_{0}}, \ldots, \rho_{x_{p}}$ соответственно образы элементов $\sigma_{x_{0}}, \ldots, \sigma_{x_{p}}$ из $\left(G_{B}\right)_{b}$ при канонических гомоморфиз$\max \left(G_{B}\right)_{\beta\left(x_{i}\right)} \rightarrow\left(G_{B}\right)_{b}(0 \leqslant i \leqslant p)$. Очевидно,что $\rho_{x_{0}} f\left(x_{0}\right), \ldots, \rho_{x_{p}} f\left(x_{p}\right)$ является $b$-репером в точке $\rho_{x_{0}} f\left(x_{0}\right)$. Но согласно (2.1.4) имеет место равенство $\rho_{x_{i}} f\left(x_{i}\right)=$ $\sigma_{x_{i}} f\left(x_{i}\right)$. Следовательно, $\sigma f\left(x_{0}\right), \ldots, \sigma f\left(x_{p}\right)$ является $b$-репером в точке $\sigma f\left(x_{0}\right)$ при $b \geqslant \beta\left(x_{0}\right), \ldots, \beta\left(x_{p}\right)$.

ОПреДЕЛЕнИЕ 3.6. Пусть $(f, \beta)$ - некоторая $A$-карта в $B$. Назовем $A$-репером в $f$ на карте $(f, \beta)$ множество таких морфизмов $f=f_{0}, f_{1}, \ldots, f_{p}: A \rightarrow B$, для которых сушествуют элементы $\sigma_{1}, \ldots, \sigma_{p} \in \prod_{x \in|A|}\left(G_{B}\right)_{\beta(x)}$, удовлетворяюшие соотношениям $\sigma_{i} f=f_{i}(1 \leqslant i \leqslant p)$. (В силу леммы 3.5 последовательность $f=f_{0}, f_{1}, \ldots, f_{p}$ является $A$-репером в любом $f_{i}$ на карте $\left(f_{i}, \beta\right)$ для $\left.0 \leqslant i \leqslant p.\right)$

В дальнейшем для нас окажется весьма полезным следующее утверждение.

ЛОКАЛЬНО-ГЛОБАЛЬНАЯ ЛЕмМА 3.7. Пусть $(f, \beta)$ - некоторая А-карта в В. Последовательность $f=f_{0}, f_{1}, \ldots, f_{p}$ является $A$-репером в $f$ на карте $(f, \beta)$ тогда и только тогда, когда для любого $x \in|A|$ последовательность $f(x), f_{1}(x)$, $\ldots, f_{p}(x)$ представляет собой локальный репер в точке $f(x)$ для $\beta(x)$.

ДокАЗАТЕЛЬствО. Это утверждение является тривиальным следствием леммы 3.5.

ОПРЕДЕЛЕНИЕ 3.8. А-нормальным морфизмом $g: B \rightarrow C$ глобальных действий называется морфизм, сохранящий $A$-реперы. Более подробно, если $f, f_{1}, \ldots, f_{p}$ - произвольньй $A$-репер в $f$ на карте $(f, \beta)$, то $g f, g f_{1}, \ldots, g f_{p}$ является $A$-репером в $g f$ на некоторой $A$-карте $(g f, \gamma)$ в $C$. Нормальнымм морфизмом $g: B \rightarrow C$ назьвается морфизм, сохраняющий $A$-реперы для любого глобального действия $A$. Наконец, $A$-нормальный (соответственно, нормальный) изоморфизм-это $A$-нормальньй (соответственно, нормальньй) морфизм, обладающий $A$-нормальным (соответственно, нормальным) обратньм.

В обшем случае $A$-нормальньй (соответственно, нормальньй) морфизм, являюшийся изоморфизмом в обычном смысле, не имеет $A$-нормального (соответственно, нормального) обратного.

Лемма 3.9. Регулярный морфизм является нормальным. 
ДокАЗАТЕЛЬСтво. Пусть $\eta: B \rightarrow C$ - регулярньй морфизм. Если $(f, \beta)-$ некоторая $A$-карта в $B$, то прямая проверка показывает, что $\left(\eta_{X} f, \eta_{\Phi} \beta\right)$ является $A$-картой в $C$. Пусть $f, f_{1}, \ldots, f_{p}$ - какой-либо $A$-репер в $f$ на карте $(f, \beta)$, а элементы $\sigma_{1}, \ldots, \sigma_{p} \in \prod_{x \in|A|}\left(G_{B}\right)_{\beta(x)}$ таковы, что $\sigma_{i} f=f_{i}(1 \leqslant i \leqslant p)$. Для $\sigma=\left(\sigma_{x}\right) \in \prod_{x \in|A|}\left(G_{B}\right)_{\beta(x)}$ положим

$$
\eta_{G}(\sigma)=\left(\eta_{G}(\beta(x))\left(\sigma_{x}\right)\right) \in \prod_{x \in|A|}\left(G_{C}\right)_{\eta_{\Phi}(\beta(x))} .
$$

Тогда в силу (3.3.4) имеет место равенство

$$
\eta_{G}\left(\sigma_{i}\right)\left(\eta_{X} f\right)=\eta_{X} f_{i}, \quad 1 \leqslant i \leqslant p .
$$

Стало быть, последовательность $\eta_{X} f, \eta_{X} f_{1}, \ldots, \eta_{X} f_{p}$ является $A$-репером в $\eta_{X} f$ на карте $\left(\eta_{X} f, \eta_{\Phi} \beta\right)$.

Зададим теперь на множестве морфизмов $\operatorname{Mor}(A, B)$ структуру глобального действия.

ОПРЕДЕЛЕНИЕ 3.10 . Пусть $A$ и $B$ - глобальные действия и $|\operatorname{Mor}(A, B)|$-множество всех морфизмов из $A$ в $B$. Обозначим через $\operatorname{Mor}(A, B)$ глобальное действие

$$
\left(\Phi_{(A, B)}, G_{(A, B)}, X_{(A, B)}\right)
$$

с обертьваюшим множеством $|\operatorname{Mor}(A, B)|$, определяемое следуюшим образом. Прежде всего, положим

$$
\Phi_{(A, B)}=\left\{\beta:|A| \rightarrow \Phi_{B}\right\}
$$

и зададим на $\Phi_{(A, B)}$ транзитивное реф̆лексивное отношение правилом

$$
\beta \leqslant \beta^{\prime} \Longleftrightarrow \beta(x) \leqslant \beta^{\prime}(x) \forall x \in|A| .
$$

Для $\beta \in \Phi_{(A, B)}$ положим

$$
\left(G_{(A, B)}\right)_{\beta}=\prod_{x \in|A|}\left(G_{B}\right)_{\beta(x)} .
$$

Если $\beta \leqslant \beta^{\prime}$, то для каждого $x \in|A|$ существует функториально определенньй гомоморфизм $\left(G_{B}\right)_{\beta(x)} \rightarrow\left(G_{B}\right)_{\beta^{\prime}(x)}$ и, следовательно, гомоморфизм $\left(G_{(A, B)}\right)_{\beta} \longrightarrow$ $\left(G_{(A, B)}\right)_{\beta^{\prime}}$, функториальньй по $\beta$. Для $\beta \in \Phi_{(A, B)}$ положим

$$
\left(X_{(A, B)}\right)_{\beta}=\{f:|A| \rightarrow|B| \mid(f, \beta) \text { есть } A \text {-карта в } B\} .
$$

Согласно лемме 3.5 , если $\sigma \in\left(G_{(A, B)}\right)_{\beta}$ и $f \in\left(X_{(A, B)}\right)_{\beta}$, то $\sigma f \in\left(X_{(A, B)}\right)_{\beta}$, следовательно, существует действие групш $\left(G_{(A, B)}\right)_{\beta}$ на $\left(X_{(A, B)}\right)_{\beta}$.

Без труда проверяется, что для $\operatorname{Mor}(A, B)$ вьполнены все аксиомы глобального действия. 
ПРЕДЛОЖЕНИЕ 3.11. Функтор $\operatorname{Mor}($, ) принимает значения в категории глобальных действий и является контравариантным и регулярным по первому аргументу относительно всех морфизмов и ковариантным по второму аргументу относительно нормальных морфизмов. Более точно, имеют место следующие утверэсдения.

(3.11.1) Пусть $C$ - глобальное действие и $f: A \rightarrow B$ - морфизм глобальных действий. Тогда $f$ определяет регулярный морфизм.

$$
\eta=\operatorname{Mor}\left(f, 1_{C}\right): \operatorname{Mor}(B, C) \rightarrow \operatorname{Mor}(A, C),
$$

который строится следующим образом. Зададим, прежсде всего, сохраняющий отношения морфизм

$$
\eta_{\Phi}: \Phi_{(B, C)} \rightarrow \Phi_{(A, C)}
$$

формулой

$$
\eta_{\Phi}(\beta)=\beta f .
$$

Далее, определим естественное преобразование

$$
\eta_{G}: G_{(B, C)} \rightarrow G_{(A, C)}
$$

Для этого достаточно определить морфизмь

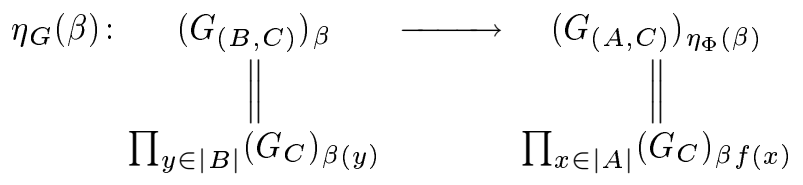

Зададим $\left.\eta_{G}(\beta)\right|_{\left(G_{C}\right)_{\beta(y)}}$ как диагональный гомоморфизм

$$
\left(G_{C}\right)_{\beta(y)} \rightarrow \prod_{x \in|A|, f(x)=y}\left(G_{C}\right)_{\beta f(x)}
$$

(пустое произведение групп, которое может появится в правой части, считается тривиальной группой). Наконеи, определим отображение

$$
\eta_{X}:|\operatorname{Mor}(B, C)| \rightarrow|\operatorname{Mor}(A, C)|,
$$

полагая

$$
\eta_{X}(g)=g f .
$$

Тогда $\eta=\left(\eta_{\Phi}, \eta_{G}, \eta_{X}\right)-$ морфизм глобальных действий.

(3.11.2) Пусть $A-$ глобальное действие и $g: B \rightarrow C-$ морфизм глобальньх действий. Тогда функиия

$$
\operatorname{Mor}\left(1_{A}, g\right):|\operatorname{Mor}(A, B)| \rightarrow|\operatorname{Mor}(A, C)|
$$

задает морфизм глобальньх действий $\operatorname{Mor}(A, B) \rightarrow \operatorname{Mor}(A, C)$ тогда и только тогда, когда морфизм $g$ является $A$-нормальньм. 
ДокАЗАТЕЛЬСтво. Утверждение (3.11.1) доказывается прямой проверкой. Детали оставляются читателю. Докажем утверждение (3.11.2). Пусть $(f, \beta)$ - некоторая $A$-карта в $B$ и $f=f_{0}, f_{1}, \ldots, f_{p}$-произвольный $A$-репер на $(f, \beta)$. Из определения вытекает, что $f_{0}, f_{1}, \ldots, f_{p}$ - локальный $\beta$-репер в глобальном действии $\operatorname{Mor}(A, B)$; обратно, любой локальньй репер в $\operatorname{Mor}(A, B)$ есть в то же время $A$-репер на некоторой $A$-карте в $B$. Следовательно, функция $\operatorname{Mor}\left(1_{A}, g\right):|\operatorname{Mor}(A, B)| \rightarrow|\operatorname{Mor}(A, C)|$ является морфизмом глобальных действий тогда и только тогда, когда она сохраняет $A$-реперы, что, в свою очередь, эквивалентно $A$-нормальности отображения $g$.

ЗАмечАниЕ. Пусть $B$ - некоторое глобальное действие. Обозначив через $\Phi_{B^{\prime}}$ подкатегорию в $\Phi_{B}$, состояшую из объектов, исчерпьваюших объекты в $\Phi_{B}$, мы получим глобальное действие $B^{\prime}=\left(\Phi_{B^{\prime}}, G_{B}, X_{B}\right)$. На первый взгляд это действие очень похоже на $B$. В самом деле, тождественное отображение $|B| \rightarrow|B|$ определяет регулярный морфизм $B^{\prime} \rightarrow B$, являющийся изоморфизмом глобальных действий. Однако, в общем случае, $B^{\prime} \rightarrow B$ не является регулярным изоморфизмом. Структурное различие между $B^{\prime}$ и $B$ можно выявить, сравнивая глобальные действия $\operatorname{Mor}\left(A, B^{\prime}\right)$ и $\operatorname{Mor}(A, B)$ с помошью канонического морфизма $\operatorname{Mor}\left(A, B^{\prime}\right) \rightarrow \operatorname{Mor}(A, B)$. Множество $A$-карт в $B^{\prime}$ в общем случае меньше, чем множество $A$-карт в $B$, вследствие чего область определения локальной группы $\left(G_{\left(A, B^{\prime}\right)}\right) \beta$ меньше области определения соответствуюшей групшы $\left(G_{(A, B)}\right)_{\beta}$, т.е. $\left(X_{\left(A, B^{\prime}\right)}\right)_{\beta} \varsubsetneqq\left(X_{(A, B)}\right)_{\beta}$.

ОПРЕДЕЛЕНИЕ 3.12. Пусть $g: B \rightarrow C$ - морфизм глобальных действий. Последовательность $A_{n}, \ldots, A_{1}$ глобальных действий назьвается нормальной цепью длины $n$ для $g$, если $g$ является $A_{1}$-нормальным и для каждого $i, 1 \leqslant i \leqslant n-1$, морфизм $\left.\operatorname{Mor}\left(1_{A_{i-1}}, \ldots, \operatorname{Mor}\left(1_{A_{1}}, g\right)\right) \ldots\right): \operatorname{Mor}\left(A_{i}, \operatorname{Mor}\left(A_{i-1}, \ldots, \operatorname{Mor}\left(A_{1}, B\right)\right) \ldots\right) \rightarrow$ $\operatorname{Mor}\left(A_{i}, \operatorname{Mor}\left(A_{i-1}, \ldots, \operatorname{Mor}\left(A_{1}, C\right)\right) \ldots\right) A_{i+1}$-нормален. Пусть $\mathscr{N}$ - некоторьй класс нормальных действий. Морфизм $g$ называется $n-\mathscr{N}$-нор мальныц, ес если любая последовательность из $n$ объектов, принадлежаших $\mathscr{N}$, образует нормальную цепь для $g$. Морфизм $g$ называется $\mathscr{N}$-нормальнылм (соответственно, $\infty-\mathscr{N}$-норммальныц), если он 1- $\mathscr{N}$-нормален (соответственно, $n$ - $\mathscr{N}$-нормален для всех $n>0$ ). Если $\mathscr{N}=\{A\}$ (соответственно, $\mathscr{N}$ состоит из всех глобальных действий), то мы будем называть морфизм глобальных действий $\infty$ - $A$-нормальныцм (соответственно, $\infty$-норммальныцм).

Если термином $t$-морфизм обозначить одно из веденных выше понятий нормальности, то $t$-изоморфизмом назьвается $t$-морфизм, обладаюший обратным $t$-морфизмом.

Чтобы связать с морфизмом $g: A \rightarrow B$ глобальных действий длинную точную последовательность алгебраических гомотопических групп, мы будем полагать, что этот морфизм $\infty$ - $L$-нормален, где $L$ - линейное действие, определенное в примере 2.4 .

Лемма 3.13. Пусть $g: B \rightarrow C$ - регулярный морфизм. Тогда для любого глобального действия $A$ морфизм

$$
\operatorname{Mor}\left(1_{A}, g\right): \operatorname{Mor}(A, B) \rightarrow \operatorname{Mor}(A, C)
$$

такаке регулярен и, следовательно, g является $\infty$-нормальным.

ДокАЗАТЕЛЬСТво. Согласно (3.9) и (3.11.2) морфизм

$$
\operatorname{Mor}\left(1_{A}, g\right): \operatorname{Mor}(A, B) \rightarrow \operatorname{Mor}(A, C)
$$

корректно определен. Пусть $\left(\eta_{\Phi}, \eta_{G}, \eta_{X}=g\right)$ - регулярная структура для $g$. Определим регулярную структуру $\left(\mu_{\Phi}, \mu_{g}, \mu_{X}=\operatorname{Mor}\left(1_{A}, g\right)\right)$ для морфизма $\operatorname{Mor}\left(1_{A}, g\right)$ следуюшим образом. 
Координатньй морфизм

$$
\mu_{\Phi}: \Phi_{(A, B)} \rightarrow \Phi_{(A, C)}
$$

зададим правилом

$$
\beta \mapsto \eta_{\Phi} \beta
$$

Определим естественное преобразование

с помощью коммутативной диаграммы

$$
\mu_{G}: G_{(A, B)} \rightarrow G_{(A, C)}
$$

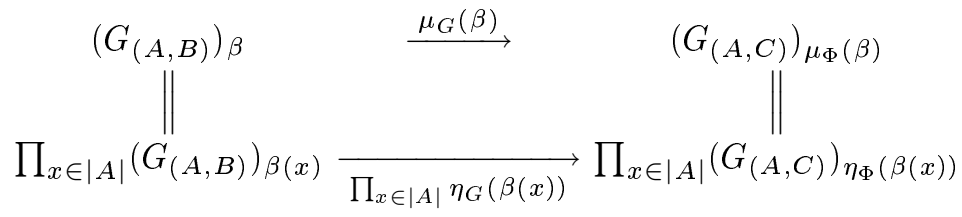

Прямая проверка показывает, что $\left(\mu_{\Phi}, \mu_{G}, \operatorname{Mor}\left(1_{A}, g\right)\right)$ - регулярньй морфизм.

Тот факт, что $g$ является $\infty$-нормальным морфизмом, получается индукцией из только что доказанного результата.

ОПРЕДЕЛЕНИЕ 3.14. Пусть $N$ - один из типов морфизмов, определенных в 3.12 $(n-\mathscr{N}$-нормальный морфизм, $\infty$-нормальньй морфизм и т. д.). Глобальное действие называется $N$-действием, если каждый его морфизм является $N$-морфизмом. Например, $\infty$-нормальное действие обладает тем свойством, что каждый его морфизм является $\infty$-нормальным.

Займемся теперь экспоненциальньм законом. Для этого нам понадобится понятие произведения глобальных действий.

ОПРЕДЕЛЕНИЕ-ЛЕММА 3.15. Пусть $A$ и В - глобальные действия. Их произведение $A \times B$ строится следуюшим образом:

$u(\alpha, \beta) \leqslant\left(\alpha^{\prime}, \beta^{\prime}\right) \Leftrightarrow \alpha \leqslant \alpha^{\prime} u \beta \leqslant \beta^{\prime} ;$

$$
\Phi_{A \times B}=\Phi_{A} \times \Phi_{B}
$$

$$
\begin{aligned}
& G_{A \times B}=G_{A} \times G_{B}, \\
& |A \times B|=|A| \times|B|, \\
& X_{A \times B}=X_{A} \times X_{B} .
\end{aligned}
$$

Для любой координать $(\alpha, \beta) \in \Phi_{A \times B}$ существует очевидное действие группь $\left(G_{A \times B}\right)_{(\alpha, \beta)}$ на $\left(X_{A \times B}\right)_{(\alpha, \beta)}$, задаваемое покомпонентно. Легко видеть, что $A \times B$ удовлетворяет универсальному свойству произведений.

Ниже мы будем использовать следуюшие обозначения. Пусть $S$ и $T$ - некоторые множества. Положим

$$
(S, T)=\operatorname{Mor}_{\text {sets }}(S, T) .
$$

Если $U$ - еше одно множество, то существует такой канонический изоморфизм множеств

$$
\begin{gathered}
E:(U,(S, T)) \stackrel{\cong}{\longrightarrow}(U \times S, T), \\
f \longmapsto E f,
\end{gathered}
$$

что $E f(u, s)=f(u)(s)$. Очевидно, что обратньм к нему является функция

$$
\begin{gathered}
E^{\prime}:(U \times S, T) \longrightarrow(U,(S, T)), \\
f \longmapsto E^{\prime} f,
\end{gathered}
$$

где $\left(E^{\prime} f(u)\right)(s)=f(u, s)$. 
ОПРеДЕЛЕНИЕ 3.17. Пусть $A, B$ и $C$ - глобальные действия. Регулярньй морфизм

$$
E: \operatorname{Mor}(A, \operatorname{Mor}(B, C)) \rightarrow \operatorname{Mor}(A \times B, C)
$$

определяется следующим образом. Обозначим структурные компонентыглобального действия $\operatorname{Mor}(A, \operatorname{Mor}(B, C))$ через $\left(\Phi_{(A,(B, C))}, G_{(A,(B, C))}, X_{(A,(B, C))}\right)$. Определим

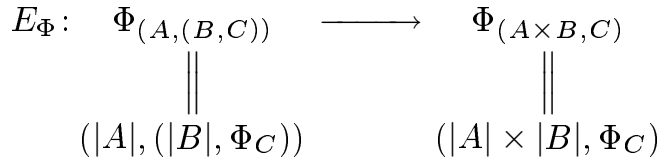

как экспоненциальный изоморфизм (3.16). Ясно, что $E_{\Phi}$ сохраняет транзитивное рефлексивное отношение. Далее, определим естественное преобразование

$$
E_{G}: G_{(A,(B, C))} \rightarrow\left(G_{(A \times B, C)}\right)_{E_{\Phi}()}
$$

так, чтобы отображение

$$
\begin{array}{rlr}
E_{G}(\alpha): & \left(G_{(A,(B, C))}\right)_{\alpha} & \stackrel{\left(G_{(A \times B, C)}\right)_{E_{\Phi}(\alpha)}}{\|} \\
\prod_{x \in|A|}\left(\prod_{y \in|B|}\left(G_{C}\right)_{\alpha(x)(y)}\right) & \prod_{(x, y) \in|A| \times|B|}\left(G_{C}\right)_{\left(E_{\Phi} \alpha\right)(x, y)}
\end{array}
$$

тождественно переводило сомножитель $\left(G_{C}\right)_{\alpha(x)(y)}$ на сомножитель $\left(G_{C}\right)_{\left(E_{\Phi} \alpha\right)(x, y)}=$ $\left(G_{C}\right)_{\alpha(x)(y)}$. Без труда проверяется, что образ композиции отображений

$$
|\operatorname{Mor}(A, \operatorname{Mor}(B, C))| \rightarrow(|A|,(|B|,|C|)) \stackrel{(3.16)}{\longrightarrow}(|A| \times|B|,|C|)
$$

лежит в $|\operatorname{Mor}(A \times B, C)|$, поэтому определим

$$
E_{X}:|\operatorname{Mor}(A, \operatorname{Mor}(B, C))| \rightarrow|\operatorname{Mor}(A \times B, C)|
$$

как результируюшее отображение. Прямые вычисления показывают, что тройка

$$
E=\left(E_{\Phi}, E_{G}, E_{X}\right)
$$

является регулярньм морфизмом. (В общем случае $E$ не является изоморфизмом (соответственно, регулярньм изоморфизмом), поскольку $E_{X}$ не обязано быть сюръективньм (соответственно, $E_{X}\left(\left(X_{(A,(B, C))}\right)_{\alpha}\right)$ не покрывают все множества $\left.\left(X_{(A \times B, C)}\right)_{E_{\Phi}(\alpha)}\right)$.)

Пусть $A_{n}, \ldots, A_{1}$ - произвольная последовательность глобальных действий. Итерируя изложенную выше процедуру, можно для любого $n \geqslant 2$ следуюшим образом получить регулярньй морфизм

$$
E_{n}: \operatorname{Mor}\left(A_{n}, \operatorname{Mor}\left(A_{n-1}, \ldots, \operatorname{Mor}\left(A_{1}, C\right)\right) \ldots\right) \rightarrow \operatorname{Mor}\left(A_{n} \times \cdots \times A_{1}, C\right) .
$$

Для $n=2$ этот морфизм был определен вьше. Предположим, что $n>2$ и что морфизм уже определен для любого натурального числа $N$, где $2 \leqslant N \leqslant n-1$. Обозначим через $E_{n-1}$ соответствуюший морфизм для последовательности $A_{n-1}, \ldots, A_{1}$. Определим теперь морфизм $E_{n}$ для последовательности $A_{n}, A_{n-1}, \ldots, A_{1}$ как композицию регулярного морфизма $\operatorname{Mor}\left(1_{A_{n}}, E_{n-1}\right)$ (см. (3.13)) и регулярного морфизма

$$
E_{2}: \operatorname{Mor}\left(A_{n}, \operatorname{Mor}\left(A_{n-1} \times \cdots \times A_{1}, B\right)\right) \rightarrow \operatorname{Mor}\left(A_{n} \times \cdots \times A_{1}, B\right) .
$$

Следуюшая конструкция поможет нам справится с проблемой нахождения обратного для морфизма $E_{n}$. 
ОПРЕДЕЛЕНИЕ 3.18. Пусть Я - некоторый класс глобальных действий, замкнутьй относительно конечньх произведений. Глобальное действие $C$ называется $\infty$ - Р-әкспоненциальнылм, если морфизм

$$
E: \operatorname{Mor}(A, \operatorname{Mor}(B, C)) \rightarrow \operatorname{Mor}(A \times B, C)
$$

является $\infty$ - $\mathscr{P}$-нормальным для всех пар $A, B \in \mathscr{P}$. Глобальное действие $C$ называется регулярно $\infty$ - $\mathscr{P}$-экспоненииальным, если $E$ - регулярный изоморфизм для всех пар $A, B \in \mathscr{P}$. Если класс $\mathscr{P}$ состоит из всех конечных произведений $A$ на себя (соответственно, $\mathscr{P}$ содержит все такие глобальные действия $A$, что $\left.|A|=\bigcup_{\alpha \in \Phi_{A}} X_{\alpha}\right)$, то $\infty$ - $\mathscr{P}$-экспоненциальное действие $C$ называется $\infty$ - $A$-экспоненииальным (соответственно, $\infty$-экспоненциальньим.

ЛЕмма 3.19. Предположим, что глобальное действие $C$ является $\infty$-Я -әкспоненциальным (соответственно, регулярно $\infty$-Я -экспоненциальным). Тогда для любой последовательности $A_{n}, \ldots, A_{1} \in \mathscr{P}$, в которой $n \geqslant 2$, морфизм $E_{n}$ из определения 3.17 является $\infty$-Я -нормальным (соответственно регулярным) изоморфизмом.

ДОкАЗАТЕльствО. Для $n=2$ утверждение следует из определений. Применяя индукцию, предположим, что утверждение справедливо для $n-1$. По определению $E_{n}=E_{2} \operatorname{Mor}\left(1, E_{n-1}\right)$. Но $E_{2}$ и $\operatorname{Mor}\left(1, E_{n-1}\right)$ суть $\infty$ - $\mathscr{P}$-изоморфизмы (соответственно, регулярные изоморфизмы). Этим завершается шаг индукции.

Следующее условие дает полезньй критерий, гарантирующий, что глобальное действие $\infty$-нормально и либо $\infty$-экспоненциально или же регулярно $\infty$-экспоненциально.

ОПРЕДЕЛЕНИЕ 3.20. Пусть $A$ - глобальное действие. Если $\Delta \subseteq \Phi_{A}$, положим

$$
\Phi_{A}^{\geqslant \Delta}=\left\{\alpha \in \Phi_{A} \mid \alpha \geqslant \beta \forall \beta \in \Delta\right\} .
$$

Назовем $A$ действием с сильной нижсей гранью, если для любого конечного подмножества $\Delta \subseteq \Phi_{A}$ и любого конечного непустого множества $U \subseteq|A|$, таких что $\left(X_{A}\right)_{\beta} \cap U \neq \varnothing$ для всех $\beta \in \Delta$, множество $\left\{\alpha \in \Phi_{A}^{\geqslant \Delta} \mid U\right.$ - некоторый $\alpha$-репер $\}$ либо пусто, либо состоит из инициального элемента. Назовем $A$ действием $с$ нижней гранью, если оно удовлетворяет приведенным выше условиям по меньшей мере для $\Delta=\varnothing$.

В следующей лемме формулируются условия, при наличии которых глобальное действие обладает сильной нижней гранью. Тем самым, доказьвается, что если финальньй объект в пространстве морфизмов является действие с нижней гранью (соответственно, сильной нижней гранью), то само пространство морфизмов наследует это свойство.

Лемма 3.21. Пусть $A$ - глобальное действие. Рассмотрим следующие условия.

(3.21.1) Пусть $\alpha, \beta \in \Phi_{A}$. Тогда $\alpha \leqslant \beta \Leftrightarrow X_{\alpha} \cap X_{\beta} \neq \varnothing$ и для всех $x \in X_{\alpha} \cap X_{\beta}$ имеет место включение $\left(G_{A}\right)_{\alpha}(x) \subseteq\left(G_{A}\right)_{\beta}(x)$.

(3.21.2) Пусть $\alpha, \beta \in \Phi_{A}$. Тогда $\alpha \leqslant \beta \Leftrightarrow X_{\alpha} \cap X_{\beta} \neq \varnothing$ и существует такое $x \in X_{\alpha} \cap X_{\beta}$, ито $\left(G_{A}\right)_{\alpha}(x) \subseteq\left(G_{A}\right)_{\beta}(x)$.

(3.21.3) Пусть $\Psi \subseteq \Phi_{A}$. Тогда для каждого $x \in \bigcap_{\alpha \in \Psi} X_{\alpha}$ найдется такое $\beta \in \Phi_{A}$, чтоо имеет место равенство $\bigcap_{\alpha \in \Psi}\left(G_{A}\right)_{\alpha}(x)=\left(G_{A}\right)_{\beta}(x)$.

Если А удовлетворяет условиям (3.21.2) и (3.21.3), то оно является действием с сильной нижней гранью. 
ДокАЗАТЕЛЬСТвО. Пусть $U$ локальный репер и $\Delta \subseteq \Phi_{A}$ - такое конечное множество, что для каждого $\delta \in \Delta$ пересечение $X_{\delta} \cap U$ не пусто. Положим $\Psi=\{\alpha \in$ $\Phi_{A} \geqslant \Delta \mid U$ - локальный $\alpha$-репер $\}$. Если $u \in U$, то $U \subseteq \bigcap_{\alpha \in \Psi}\left(G_{A}\right)_{\alpha}(u)=\left(G_{A}\right)_{\beta}(u)$ для некоторого $\beta \in \Phi_{A}$ в силу условия (3.21.3). Очевидно, что $U$ является локальным $\beta$-репером. Так как $\left(G_{A}\right)_{\beta}(u) \subseteq\left(G_{A}\right)_{\alpha}(u)$, то из (3.21.2) вытекает, что $\beta \leqslant \alpha$ для всех $\alpha \in \Psi$. Поэтому, если $\Delta=\varnothing$, то все доказано. Если же $\Delta \neq \varnothing$, мы должны доказать, что $\delta \leqslant \beta$ для любого $\delta \in \Delta$. Пусть $u \in X_{\delta} \cap U$. Так как $\delta \leqslant \alpha$ для любого $\alpha \in \Psi$, то из (3.21.2) следует, что $\left(G_{A}\right)_{\delta}(u) \subseteq \bigcap_{\alpha \in \Psi}\left(G_{A}\right)_{\alpha}(u)=\left(G_{A}\right)_{\beta}(u)$. Это означает, что $\delta \leqslant \beta$.

ЗАмЕчАнИЕ. В то время как симплициальные действия $\operatorname{gl}(S)$ и $f \operatorname{gl}(S)$ из $(2.5)$ и произвольная модель Володина (2.3) удовлетворяют условию сильной нижней грани, только лишь модель Володина удовлетворяет условиям доказанной вьше леммы.

Лемма 3.22. Пусть В - действие с нижней гранью (соответственно, сильной нижней гранью). Тогда для любого глобального действия $A$ функтор $\operatorname{Mor}(A, B)$ также является действием с нижней гранью (соответственно, $c$ сильной нижней гранью).

ДокаЗАТЕЛЬСтво. Пусть $U \subseteq|\operatorname{Mor}(A, B)|$ - конечное непустое подмножество. Рассмотрим такое конечное подмножество $\Delta \subseteq \Phi_{(A, B)}$, что для каждого $\delta \in \Delta$ пересечение $\left(X_{(A, B)}\right)_{\delta} \cap U$ не пусто. Положим $\Psi=\left\{\beta \in \Phi_{(A, B)} \mid U\right.$ - некоторый $\beta$-репер, $\delta \leqslant \beta \forall \delta \in \Delta\}$ и будем считать, что $\Psi \neq \varnothing$. Мы должны показать, что $\Psi$ имеет инициальньй элемент. С этой целью положим для каждого $x \in|A|$

$$
U(x)=\{f(x) \mid f \in U\}, \quad \Delta(x)=\{\delta(x) \mid \delta \in \Delta\}, \quad \Psi(x)=\{\beta(x) \mid \beta \in \Psi\} .
$$

По предположению для каждого $x \in|A|$ найдется такая координата $b_{x} \in \Phi_{B}$, что $U(x)$ является $b_{x}$-репером и $\delta(x) \leqslant b_{x} \leqslant \beta(x)$ для всех $\delta \in \Delta$ и $\beta \in \Psi$. Рассмотрим отображение $\gamma:|A| \rightarrow \Phi_{B}, x \mapsto b_{x}$. Ясно, что $\delta \leqslant \gamma \leqslant \beta$ для всех $\delta \in \Delta$ и $\beta \in \Psi$. Пусть $u \in U$. Чтобы завершить доказательство, достаточно показать, что пара $(u, \gamma)$ является $A$-картой. Возьмем некоторый элемент $\beta \in \Psi$. Так как пара $(u, \beta)$ является $A$-картой и $\gamma \leqslant \beta$, то очевидно, что $(u, \gamma)$ - также $A$-карта.

Следуюшая теорема является главньм результатом работы.

ТЕОРема 3.23. Любое действие с нижней гранью является $\infty$-нормальнымм $u \infty$-экспоненциальным. Действие с сильной нижней гранью является $\infty$-нормальным и регулярно $\infty$-әкспоненииальныцм.

В доказательстве теоремы 3.23 несколько раз будет использоваться следующая лемма.

Лемма 3.24. Пусть $A$ и $B$ - глобальные действия, $(f, \beta)$ - некоторая $A$-карта в B и $f=f_{0}, f_{1}, \ldots, f_{p}$ - некоторый $A$-репер на карте $(f, \beta)$. Если $x_{0}, \ldots, x_{q} \in|A|$ - локальный репер в $A$, то множество $\left\{f_{i}\left(x_{j}\right) \mid 0 \leqslant i \leqslant p, 0 \leqslant j \leqslant q\right\}$ является локальныцм b-репером для некоторого $b \in \Phi_{B}$ такого, что $b \geqslant \beta_{x_{0}}, \ldots, \beta_{x_{q}}$.

ДоказАтельство. Так как $\left(f_{0}, \beta\right)$ является $A$-картойв $B$, то из определения вытекает, что множество $f_{0}\left(x_{0}\right), \ldots, f_{0}\left(x_{q}\right)$ есть локальньй $b$-репер для некоторого $b \in \Phi_{B}$, удовлетворяюшего условию $b \geqslant \beta_{x_{0}}, \ldots, \beta_{x_{q}}$. Стало быть, группа $\left(G_{B}\right)_{b}$ транзитивно действует на $f_{0}\left(x_{0}\right), \ldots, f_{0}\left(x_{q}\right)$. Чтобы завершить доказательство, достаточно показать, что $\left(G_{B}\right)_{b}$ транзитивно действует на $\left\{f_{i}\left(x_{j}\right) \mid 0 \leqslant i \leqslant p, 0 \leqslant j \leqslant q\right\}$. Поскольку $f_{0}, \ldots, f_{p}$ является $A$-репером на карте $(f, \beta)$, группа $\left(G_{(A, B)}\right)_{\beta}$ действует на нем 
транзитивно. Поэтому для любого $x \in|A|$ групп $\left(G_{B}\right)_{\beta_{x}}$ транзитивно действует на $f_{0}(x), \ldots, f_{p}(x)$. Используя канонический гомоморфизм $\left(G_{B}\right)_{\beta_{x_{j}}} \rightarrow\left(G_{B}\right)_{b}$ и замечая, что $f_{0}\left(x_{j}\right) \in\left(X_{B}\right)_{b}$, мы заключаем, что группа $\left(G_{B}\right)_{b}$ транзитивно действует на $f_{0}\left(x_{j}\right), \ldots, f_{p}\left(x_{j}\right)$. Так как это имеет место для каждого $j,: 0 \leqslant j \leqslant q$, а группа $\left(G_{B}\right)_{b}$ транзитивно действует на $f_{0}\left(x_{0}\right), \ldots, f_{p}\left(x_{q}\right)$, то она транзитивно действует и на $\left\{f_{i}\left(x_{j}\right) \mid 0 \leqslant i \leqslant p, 0 \leqslant j \leqslant q\right\}$.

ДОКАЗАТЕЛЬСТВо ТЕОРЕМЫ 3.23. Пусть $C$ - действие с нижней гранью. Покажем, что $C$ является $\infty$-нормальным. В силу леммы 3.22 мы должны доказать, что $C$ является $A$-нормальным для любого глобального действия $A$. Пусть $g: B \rightarrow C-$ морфизм глобальных действий, $(f, \beta)$ - некоторая $A$-карта в $B$ и $f=f_{0}, f_{1}, \ldots, f_{p}-$ произвольный репер на $(f, \beta)$. Покажем, что $g f_{0}, \ldots, g f_{p}$ является $A$-репером в $C$.

Построим, прежде всего, такую координату $\left(\gamma:|A| \rightarrow \Phi_{C}\right) \in \Phi_{(A, C)}$, что $(g f, \gamma)$ есть $A$-карта в $C$. Для $x \in|A|$ положим $U(x)=\left\{g f_{0}(x), \ldots, g f_{p}(x)\right\}$. Согласно локально-глобальной лемме 3.7 набор $f_{0}(x), \ldots, f_{p}(x)$ является локальным репером в $B$. Поскольку $g$ - морфизм, отсюда следует, что $U(x)$ - локальный репер в $C$. Так как $C$ обладает нижней гранью, то множество $\Psi(x)=\left\{c \in \Phi_{C} \mid U(x)\right.$ есть $c$-репер $\}$ имеет инициальньй элемент $c_{x}$. Определим $\gamma:|A| \rightarrow \Phi_{C}$ полагая $\gamma(x)=c_{x}$. Проверим теперь, что $(g f, \gamma)$ есть $A$-карта в $C$. Пусть $x_{0}, \ldots, x_{q}$ - некоторьй локальный репер в $A$. Согласно лемме 3.24 множество $\left\{f_{i}\left(x_{j}\right) \mid 0 \leqslant i \leqslant p, 0 \leqslant j \leqslant q\right\}$ представляет собой локальный репер в $B$. Стало быть, множество $\left\{g f_{i}\left(x_{j}\right) \mid 0 \leqslant i \leqslant p, 0 \leqslant j \leqslant q\right\}$ является локальным $c$-репером для некоторого $c \in \Phi_{C}$. Ясно, что $\gamma\left(x_{j}\right)=c_{x_{j}} \leqslant c$, поскольку $c_{x_{j}}$ - инициальньй элемент в $\Psi\left(x_{j}\right)$. А это означает, что $(g f, \gamma)$ есть $A$-карта в $C$. По лемме 3.7 множество $f_{0}, \ldots, f_{p}$ является $A$-репером на $(f, \gamma)$ тогда и только тогда, когда $f_{0}(x), \ldots, f_{p}(x)$ - локальный $\gamma(x)$-репер для всех $x \in|A|$. Так как это свойство вытекает их определения $\gamma(x)$, то тем самым мы доказали, что глобальное действие $C$ является $A$-нормальным.

Пусть снова $C$ - действие с нижней гранью. Покажем теперь, что $C$ является $\infty$-экспоненциальным. Рассмотрим такие глобальные действия $A$ и $B$, что $|A|=\bigcup_{\alpha \in \Phi_{A}}\left(X_{A}\right)_{\alpha}$ и $|B|=\bigcup_{\beta \in \Phi_{B}}\left(X_{B}\right)_{\beta}$. Пусть $E: \operatorname{Mor}(A, \operatorname{Mor}(B, C)) \rightarrow$ $\operatorname{Mor}(A \times B, C)$ - морфизм из определения 3.17. Докажем, что $E$ имеет $\infty$-нормальньй обратньй. Согласно лемме $3.22 \operatorname{Mor}(A, \operatorname{Mor}(B, C))$ является действием с нижней гранью и поэтому, как мы только что показали, оно должно быть $\infty$-нормальньм. Следовательно, если у $E$ существует обратньй морфизм, то он обязательно будет $\infty$-нормальньм. Поэтому нам достаточно доказать, что морфизм $E$ обладает обратным. Имеется очевидньй кандидат для такого обратного морфизма, а именно теоретико-множественное отображение

$$
E^{\prime}:|\operatorname{Mor}(A \times B, C)| \rightarrow(A,(B, C)), \quad f \mapsto E^{\prime} f
$$

где $\left(E^{\prime} f(x)\right)(y)=f(x, y)$. Покажем, что $E^{\prime} f \in|\operatorname{Mor}(A, \operatorname{Mor}(B, C))|$ и что результируюшее отображение

$$
E^{\prime}:|\operatorname{Mor}(A \times B, C)| \rightarrow|\operatorname{Mor}(A, \operatorname{Mor}(B, C))|
$$

определяет морфизм $\operatorname{Mor}(A \times B, C) \rightarrow \operatorname{Mor}(A, \operatorname{Mor}(B, C))$ глобальных действий.

Из теоретико-множественного определения отображения $E^{\prime}$ очевидно, что $E^{\prime}$ будет обратным для $E$. Покажем, что $E^{\prime} f:|A| \rightarrow(B, C)$ определяет морфизм $A \rightarrow$ $\operatorname{Mor}(B, C)$ глобальных действий. Для этого нам нужно проверить следующие свойства:

(i) если $x \in|A|$, то отображение $E^{\prime} f(x):|B| \rightarrow|C|, y \mapsto\left(E^{\prime} f(x)\right)(y)$ задает морфизм глобальных действий $B \rightarrow C$; 
(ii) результирующее отображение $E^{\prime} f:|A| \rightarrow|\operatorname{Mor}(B, C)|, x \mapsto E^{\prime} f(x)$ задает морфизм глобальных действий $A \rightarrow \operatorname{Mor}(B, C)$.

Пусть $x \in|A|$ и $y_{0}, \ldots, y_{q}$ - некоторый локальньй репер в $B$. Так как $|A|=\bigcup_{\alpha \in \Phi_{A}}\left(X_{A}\right)_{\alpha}$, то $x$ - локальный репер в $A$ и, стало быть, $\left(x, y_{0}\right), \ldots,\left(x, y_{q}\right)$ - локальньй репер в $A \times B$. Следовательно, $f\left(x, y_{0}\right), \ldots, f\left(x, y_{q}\right)$ - локальньй репер в $C$. Поскольку $f\left(x, y_{j}\right)=\left(E^{\prime} f(x)\right)\left(y_{j}\right), 0 \leqslant j \leqslant q$, мы получаем, что $\left(E^{\prime} f(x)\right)\left(y_{0}\right), \ldots,\left(E^{\prime} f(x)\right)\left(y_{q}\right)$ - локальный репер в $C$. А это означает, что $E^{\prime} f(x): B \rightarrow C$ - морфизм глобальных действий.

Рассмотрим теперь в $A$ локальньй репер $x_{0}, \ldots, x_{p}$ и покажем, что $E^{\prime} f\left(x_{0}\right), \ldots$, $E^{\prime} f\left(x_{p}\right)$ - локальньй репер в $\operatorname{Mor}(B, C)$. Каждый элемент $y \in|B|$ является локальньм репером в $B$, поскольку $|B|=\bigcup_{\beta \in X_{B}}\left(X_{B}\right)_{\beta}$. Следовательно, $\left(x_{0}, y\right), \ldots,\left(x_{p}, y\right)$ - локальньй репер в $A \times B$ и $f\left(x_{0}, y\right), \ldots, f\left(x_{p}, y\right)$ локальньй репер в $C$. Так как $C$ обладает нижней гранью, то множество $\left\{c \in \Phi_{C} \mid f\left(x_{0}, y\right), \ldots, f\left(x_{p}, y\right)\right.$ является $c$-репером $\}$ имеет инициальньй элемент $c_{y}$. Определим отображение $\gamma:|B| \rightarrow \Phi_{C}$ полагая $\gamma(y)=c_{y}$. Покажем, что $\left(E^{\prime} f\left(x_{0}\right), \gamma\right)$ является $B$-картой в $C$. Предположив на мгновение, что это доказано, мы получим из локально-глобальной леммы 3.7 , что множество $E^{\prime} f\left(x_{0}\right), \ldots, E^{\prime} f\left(x_{p}\right)$ является $B$-репером на $\left(E^{\prime} f\left(x_{0}\right), \gamma\right)$. Но тогда, по определению, $E^{\prime} f\left(x_{0}\right), \ldots, E^{\prime} f\left(x_{p}\right)$ локальньй репер в $\operatorname{Mor}(B, C)$, а это - как раз то свойство, которое мы доказьвали.

Итак, покажем, что $\left(E^{\prime} f\left(x_{0}\right), \gamma\right)$ является $B$-картой в $C$. Пусть $y_{0}, \ldots, y_{q}-$ некоторьй локальньй репер в $B$. Нам нужно доказать, что множество $\left(E^{\prime} f\left(x_{0}\right)\right)\left(y_{0}\right), \ldots$, $\left(E^{\prime} f\left(x_{0}\right)\right)\left(y_{q}\right)$ является локальным $c$-репером для такой координаты $c \in \Phi_{C}$, что $c \geqslant$ $\gamma\left(y_{0}\right), \ldots, \gamma\left(y_{q}\right)$. Так как $x_{0}, \ldots, x_{p}$ - локальньй репер в $A$ и $y_{0}, \ldots, y_{q}$ - локальный репер в $B$, то множество $\left\{\left(x_{i}, y_{j}\right) \mid 0 \leqslant i \leqslant p, 0 \leqslant j \leqslant q\right\}$ является локальным репером в $A \times B$. Следовательно, $\left\{f\left(x_{i}, y_{j}\right) \mid 0 \leqslant i \leqslant p, 0 \leqslant j \leqslant q\right\}-$ локальньй $c$-репер для некоторого $c \in \Phi_{C}$. Но по определению функции $\gamma$ мы имеем $c \geqslant \gamma\left(y_{j}\right)$ для всех $j$, $0 \leqslant j \leqslant q$.

Докажем теперь, что функция

$$
E^{\prime}:|\operatorname{Mor}(A \times B, C)| \rightarrow|\operatorname{Mor}(A, \operatorname{Mor}(B, C))|
$$

задает морфизм $\operatorname{Mor}(A \times B, C) \rightarrow \operatorname{Mor}(A, \operatorname{Mor}(B, C))$ глобальных действий. Пусть $f=f_{0}, f_{1}, \ldots, f_{p} \in|\operatorname{Mor}(A \times B, C)|$ - локальный репер в $\operatorname{Mor}(A \times B, C)$. Мы должны показать, что $E^{\prime} f_{0}, \ldots, E^{\prime} f_{p}$ является локальным репером в $\operatorname{Mor}(A, \operatorname{Mor}(B, C))$. Для каждого элемента $(x, y)$ из $A \times B$ множество $f_{0}(x, y), \ldots, f_{p}(x, y)$ в силу леммы 3.7 является локальным репером в $C$. Так как $C$ обладает нижней гранью, то множество $\left\{c \in \Phi_{C} \mid f_{0}(x, y), \ldots, f_{p}(x, y)\right.$ есть $c$-репер $\}$ имеет инициальньй элемент $c_{(x, y)}$. Определим отображение $\gamma:|A| \rightarrow\left(|B|, \Phi_{C}\right)$, полагая $\gamma(x)=c_{\left(x,{ }_{-}\right)}$. Мы утверждаем, что $\left(E^{\prime} f_{0}, \gamma\right)$ является $A$-картой в $\operatorname{Mor}(B, C)$. В самом деле, из определения $\gamma$ и леммы 3.7 следует, что множество $E^{\prime} f_{0}, \ldots, E^{\prime} f_{p}$ является $A$-репером на $\left(E^{\prime} f, \gamma\right)$. Но это означает, что $E^{\prime} f_{0}, \ldots, E^{\prime} f_{p}$ - локальный репер в $\operatorname{Mor}(A, \operatorname{Mor}(B, C))$, и следовательно, $E^{\prime}$ задает морфизм глобальных действий $\operatorname{Mor}(A \times B, C) \rightarrow \operatorname{Mor}(A, \operatorname{Mor}(B, C))$.

Займемся теперь доказательством того, что $\left(E^{\prime} f, \gamma\right)$ является $A$-картой. Пусть $x_{0}, \ldots, x_{q}$ - локальный репер в $A$. Мы должны показать, что $E^{\prime} f\left(x_{0}\right), \ldots E^{\prime} f\left(x_{q}\right)$ является локальным $\delta$-репером в $\operatorname{Mor}(B, C)$ для некоторого $\delta:|B| \rightarrow \Phi_{C}$, удовлетворяюшего условиям $\delta \geqslant \gamma\left(x_{0}\right), \ldots, \gamma\left(x_{q}\right)$. Так как $f=f_{0}, f_{1}, \ldots, f_{p}$ - локальньй репер в $\operatorname{Mor}(A \times B, C)$, то в $C$ найдется такая $A \times B$-карта $\left(f_{0}, \varepsilon\right)$, что $f_{0}, \ldots, f_{p}$ является $A \times B$-репером на $\left(f_{0}, \varepsilon\right)$. Для любого фиксированного $y \in|B|$ пара $\left(f_{0}(, y), \varepsilon(, y)\right)$ является $A$-картой в $C$, а $f_{0}(, y), \ldots, f_{p}(, y)-A$-репером на $\left(f_{0}(, y), \varepsilon(, y)\right)$. Поскольку 
$x_{0}, \ldots, x_{q}$ - локальный репер в $A$, из леммы 3.24 вытекает, что множество $\left\{f_{i}\left(x_{j}, y\right) \mid\right.$ $0 \leqslant i \leqslant p, 0 \leqslant j \leqslant q\}$ является локальным репером. Так как $C$ обладает нижней гранњю, то множество $\left\{c \in \Phi_{C} \mid\left\{f_{i}\left(x_{j}, y\right) \mid 0 \leqslant i \leqslant p, 0 \leqslant j \leqslant q\right\}\right.$ - локальньй репер в $\left.C\right\}$ имеет инициальньй элемент $d_{y}$. Ясно, что $d_{y} \geqslant\left(\gamma\left(x_{j}\right)\right)(y)(0 \leqslant j \leqslant q)$. Определим отображение $\delta:|B| \rightarrow \Phi_{C}$ полагая $\delta(y)=d(y)$. В силу сказанного $\delta \geqslant \gamma\left(x_{j}\right)$ $(0 \leqslant j \leqslant q)$. Поскольку $\left(F f\left(x_{j}\right)\right)(y)=f_{0}\left(x_{j}, y\right)(0 \leqslant j \leqslant q)$ и множество $\left\{f_{i}\left(x_{j}, y\right)\right.$ $0 \leqslant i \leqslant p, 0 \leqslant j \leqslant q\}$ является $\delta(y)$-репером, то $\left(F f\left(x_{0}\right)\right)(y), \ldots,\left(F f\left(x_{q}\right)\right)(y)$ - также $\delta(y)$-репер. Поэтому, согласно лемме 3.7 множество $F f\left(x_{0}\right), \ldots, F f\left(x_{q}\right)$ является репером на $\left(F f\left(x_{0}\right), \delta\right)$, в предположении, что $\left(F f\left(x_{0}\right), \delta\right)$ есть $B$-карта в $C$. Доказательством этого факта мы сейчас и займемся.

Пусть $y_{0}, \ldots, y_{r}$ - некоторый локальньй репер в $B$. Мы должны показать, что множество $\left(F f\left(x_{0}\right)\right)\left(y_{0}\right), \ldots,\left(F f\left(x_{0}\right)\right)\left(y_{r}\right)$ является $c$-репером для некоторого $c \in \Phi_{C}$, удовлетворяющего условиям $c \geqslant \delta\left(y_{0}\right), \ldots, \delta\left(y_{r}\right)$. Так как множество $\left\{\left(x_{j}, y_{k}\right) \mid 0 \leqslant j \leqslant q, 0 \leqslant k \leqslant r\right\}$ есть локальный репер в $A \times B$ и $f_{0}, \ldots, f_{p}$ есть $A \times B$-репер в $C$, то из леммы 3.24 следует, что множество $\left\{f_{i}\left(x_{j}, y_{k}\right)\right.$ $0 \leqslant i \leqslant p, 0 \leqslant j \leqslant q, 0 \leqslant k \leqslant r\}$ является $c$-репером для некоторого $c \in \Phi_{C}$. Из определения отображения $\delta$ очевидно, что $c \geqslant \delta\left(y_{0}\right), \ldots, \delta\left(y_{r}\right)$. Поскольку $\left(F f\left(x_{0}\right)\right)\left(y_{k}\right)=f_{0}\left(x_{0}, y_{k}\right)(0 \leqslant k \leqslant r)$ и $\left\{f_{i}\left(x_{j}, y_{k}\right) \mid 0 \leqslant i \leqslant p, 0 \leqslant j \leqslant q, 0 \leqslant k \leqslant r\right\}$ есть $c$-репер, ясно, что $\left(F f\left(x_{0}\right)\right)\left(y_{0}\right), \ldots,\left(F f\left(x_{0}\right)\right)\left(y_{r}\right)$ - также $c$-репер. Этим завершается доказательство $\infty$-экспоненщиальности глобального действия $C$.

Наконец, предположим, что $C$ - глобальное действие с сильной нижней гранью. Мы должны доказать, что $C$ регулярно $\infty$-экспоненциальное действие. Для этого достаточно показать, что определенньй вьше морфизм

$$
E: \operatorname{Mor}(A, \operatorname{Mor}(B, C)) \rightarrow \operatorname{Mor}(A \times B, C)
$$

имеет регулярный обратньй морфизм $E^{\prime}$. Существуют очевидные кандидаты для компонент $\left(E_{\Phi}^{\prime}, E_{G}^{\prime}, E_{X}^{\prime}\right)$ этого морфизма. Прежде всего отображение

$$
E_{X}^{\prime}: \operatorname{Mor}(A \times B, C)|\longrightarrow| \operatorname{Mor}(A, \operatorname{Mor}(B, C)) \mid
$$

можно задать формулой

$$
E_{X}^{\prime} f=E^{\prime} f
$$

где отображение $f \mapsto E^{\prime} f$ было определено вьше.

Определим теперь

$$
E_{\Phi}^{\prime}: \Phi_{(A \times B, C)} \longrightarrow \Phi_{(A,(B, C))}
$$

как отображение множеств, обратное к $E_{\Phi}$ (см. (3.16)). И, наконец, определим естественное преобразование

$$
E_{G}^{\prime}: G_{(A \times B, C)} \longrightarrow\left(G_{(A,(B, C))}\right)_{E_{\Phi}^{\prime}()}
$$

так, чтобы

$$
\begin{aligned}
E_{G}^{\prime}(\alpha): \underset{(A \times B, C)}{\left(G_{\alpha}\right.} & \left(G_{(A,(B, C))}\right)_{E_{\Phi}^{\prime}(\alpha)} \\
\prod_{(x, y) \in|A| \times|B|}\left(G_{C}\right)_{\alpha(x, y)} & \prod_{x \in|A|}\left(\prod_{y \in|B|}\left(G_{C}\right)_{E_{\Phi}^{\prime}(\alpha)(x)(y)}\right)
\end{aligned}
$$

тождественно отображало сомножитель $\left(G_{C}\right)_{\alpha(x, y)}$ на сомножитель $\left(G_{C}\right)_{E_{\Phi}^{\prime}(\alpha)(x)(y)}$ $=\left(G_{C}\right)_{\alpha(x, y)}$. Если $E^{\prime}-$ регулярньй морфизм, то из построения очевидно, что он является регулярным обратным для регулярного морфизма $E$. 
Из свойств, которые нужно проверить для доказательства регулярности $E^{\prime}$, не очевидным, пожалуй, является лиш включение

$$
E_{X}^{\prime}\left(X_{(A \times B, C)}\right)_{\alpha} \subseteq\left(X_{(A,(B, C))}\right)_{E_{\Phi}^{\prime}(\alpha)}, \quad \alpha \in \Phi_{(A \times B, C)} .
$$

Для доказательства этого включения достаточно показать, что если $(f, \alpha)-$ некоторая $(A \times B)$-карта в $C$, то $\left(E_{X}^{\prime}(f), E_{\Phi}^{\prime}(\alpha)\right)$ является $A$-картой в $\operatorname{Mor}(B, C)$. Пусть $x_{0}, \ldots, x_{p}$ - некоторьй локальньй репер в $A$. Мы должны показать, что $E_{X}^{\prime}(f)\left(x_{0}\right), \ldots, E_{X}^{\prime}(f)\left(x_{p}\right)$ является $\gamma$-репером в $\operatorname{Mor}(B, C)$ для некоторого $\gamma:|B| \rightarrow \Phi_{C}$, удовлетворяюшего условиям $\gamma \geqslant E_{\Phi}^{\prime}(\alpha)\left(x_{i}\right)(0 \leqslant i \leqslant p)$. Для любого $y \in|B|$ элементы $\left(x_{0}, y\right), \ldots,\left(x_{p}, y\right)$ образуют локальный репер в $A \times B$. Стало быть, $f\left(x_{0}, y\right), \ldots, f\left(x_{p}, y\right)$ - локальньй репер в $C$. Из сушествования у глобального действия $C$ сильной нижней грани вытекает, что множество $\left\{c \in \Phi_{C} \mid\right.$ $f\left(x_{0}, y\right), \ldots, f\left(x_{p}, y\right)$ есть $c$-репер, $\left.c \geqslant E_{\Phi}^{\prime}\left(x_{i}\right)(y)(0 \leqslant i \leqslant p)\right\}$ имеет инищиальный элемент $c_{y}$. Определим отображение $\gamma:|B| \rightarrow \Phi_{C}$, полагая $\gamma(y)=c_{y}$. Ясно, что $\gamma \geqslant E_{\Phi}^{\prime}(\alpha)\left(x_{i}\right)(0 \leqslant i \leqslant p)$. Мы должны показать, что $\left(E_{X}^{\prime}(f)\left(x_{0}\right), \gamma\right)$ есть $B$-карта в $C$. Предположим, что это сделано. Тогда из леммы 3.7 и из того факта, что $E_{X}^{\prime}(f)\left(x_{0}\right)(y), \ldots, E_{X}^{\prime}(f)\left(x_{p}\right)(y)$ является $\gamma(y)$-репером для любого $y \in|B|$ следует, что $E_{X}^{\prime}(f)\left(x_{0}\right), \ldots, E_{X}^{\prime}(f)\left(x_{p}\right)$ есть $B$-репер на карте $\left(E_{X}^{\prime}(f)\left(x_{0}\right), \gamma\right)$. Но это означает, что $E_{X}^{\prime}(f)\left(x_{0}\right), \ldots, E_{X}^{\prime}(f)\left(x_{p}\right)$ является $\gamma$-репером в $\operatorname{Mor}(B, C)$ и теорема, тем самым, полностью доказана.

Итак, нам осталось показать, что $\left(E_{X}^{\prime}(f)\left(x_{0}\right), \gamma\right)$ есть $B$-карта в $C$. Пусть $y_{0}, \ldots, y_{q}$ - некоторьй локальный репер в $B$. Тогда множество $\left\{\left(x_{i}, y_{j}\right) \mid 0 \leqslant i \leqslant p, 0 \leqslant j \leqslant q\right\}$ есть локальньй репер в $A \times B$. Следовательно, множество $\left\{f\left(x_{i}, y_{j}\right) \mid 0 \leqslant i \leqslant p\right.$, $0 \leqslant j \leqslant q\}$ является локальньм $c$-репером для некоторого $c \in \Phi$, удовлетворяюшего условиям $c \geqslant \alpha\left(x_{i}, y_{j}\right)=E_{\Phi}^{\prime}(\alpha)\left(x_{i}\right)\left(y_{j}\right)(0 \leqslant i \leqslant p, 0 \leqslant j \leqslant q)$. Так как $E_{X}^{\prime}(f)\left(x_{0}\right)\left(y_{j}\right)=f\left(x_{0}, x_{j}\right)(0 \leqslant j \leqslant q)$, то очевидно, что множество $E_{X}^{\prime}(f)\left(x_{0}\right)\left(y_{0}\right)$, $\ldots, E_{X}^{\prime}(f)\left(x_{0}\right)\left(y_{q}\right)$ является $c$-репером и $c \geqslant E_{\Phi}^{\prime}(\alpha)\left(x_{0}\right)\left(y_{j}\right)(0 \leqslant j \leqslant q)$.

\section{4. Относительные действия и их пространства морфизмов}

Для того чтобы развить гомотопическую теорию глобальных действий, нам понадобятся пунктированные действия и, более общо, относительные действия. В этом параграфе мы введем соответствующие понятия и определения. Заметим, что относительные действия устроены более сложно, чем их топологические двойники, и поэтому при работе с ними требуется определенная осторожность. Главньй результат параграфа - это относительный вариант экспоненциального закона, доказанного в теореме 3.23 .

ОПРЕДЕЛЕНИЕ 4.1. Пусть $A$-глобальное действие. Поддействие в $A$ - это такое глобальное действие $B$, что $|B| \subseteq|A|$, причем указанное включение индуцирует морфизм глобальных действий $B \rightarrow A$. Если $B$ - поддействие в $A$, то мы обозначаем этот факт как $B \subseteq A$. Пусть $n \in \mathbb{N} \cup\{\infty\}$ и $\mathscr{N}-$ некоторый класс глобальных действий. Поддействие $B \subseteq A$ назьвается $n$ - $\mathscr{N}$-нормальным (соответственно, регулярныцм), если канонический морфизм $B \rightarrow A$ является $n$ - $\mathscr{N}$-нормальным (соответственно, регулярным). Собственное поддействие - это такое регулярное поддействие, что канонический морфизм $\Phi_{B} \rightarrow \Phi_{A}$ инъективен. Стандартнылм поддействием назьвается такое собственное поддействие $B \subseteq A$, что $\Phi_{B}=\left\{\alpha \in \Phi_{A}\left|\left(X_{A}\right)_{\alpha} \cap\right| B \mid \neq \varnothing\right\}$, $\Phi_{B}$ - полная подкатегория в $\Phi_{A}$ и для всех $\alpha \in \Phi_{B}$ имеют место равенства $\left(X_{B}\right)_{\alpha}=$ $\left(X_{A}\right)_{\alpha} \cap|B|,\left(G_{B}\right)_{\alpha}=\operatorname{Stab}_{G_{A}}\left(\left(X_{B}\right)_{\alpha}\right):=\left\{\sigma \in\left(G_{A}\right)_{\alpha} \mid \sigma\left(X_{B}\right)_{\alpha}=\left(X_{B}\right)_{\alpha}\right\}$.

Имеет место следуюшее очевидное утверждение. 
ЛЕмма 4.2. Пусть $A$ - глобальное действие. Если $Y \subseteq|A|$, то существует и единственно такое глобальное действие $B$, что $|B|=Y$ и $B$ является стандартным поддействием в $A$.

ОПреДЕЛЕНИЕ 4.3. Пусть $A$ - глобальное действие. Отмеченной точкой для $A$ называется такое поддействие $B \subseteq A$, что $|B|$ содержит ровно одну точку. Любая отмеченная точка является $\infty$-нормальньм поддействием. Отмеченная точка назьвается регулярной, собственной, или стандартной, если таковьм является соответствующее поддействие.

ОПРЕДЕЛЕНИЕ 4.4. Относительное глобальное действие - это такая упорядоченная пара $A=\left(A^{(1)}, A^{(2)}\right)$ глобальных действий, что $A^{(2)}$ есть $\infty$-нормальное поддействие в $A^{(1)}$. Относительное глобальное действие $A$ назьвается регулярнылм, собственным, или стандартным, если таковым является соответствуюшее поддействие $A^{(2)} \subseteq A^{(1)}$.

ОПРЕДЕЛЕНИЕ 4.5. Пунктированное глобальное действие - это такое относительное глобальное действие $A=\left(A^{(1)}, A^{(2)}\right)$, что $A^{(2)}$ - отмеченная точка для $A^{(1)}$. Оно называется регулярнылм, собственнылм, или стандартным, если такова его отмеченная точка.

ОПРЕДЕЛЕНИЕ 4.6. Морфизм относительных действий $f: A \rightarrow B$ - это такой морфизм глобальных действий $f: A^{(1)} \rightarrow B^{(1)}$, что $\left|A^{(2)}\right|$ переходит в $\left|B^{(2)}\right|$ и индуцирует морфизм $A^{(2)} \rightarrow B^{(2)}$. Морфизм относительных действий $f: A \rightarrow B$ называется регулярнылм, если морфизмы $f: A^{(i)} \rightarrow B^{(i)}, i=1,2$, глобальных действий несут фиксированную регулярную структуру.

ОПРЕДЕЛЕНИЕ 4.7. Пусть $A$ - относительное действие. Пара $U=\left(U^{(1)}, U^{(2)}\right)$ локальных реперов состоит из таких локальных реперов $U^{(i)}$ в $A^{(i)}, i=1,2$, что $U^{(2)} \subseteq U^{(1)}$. Пара $\left(U^{(1)}, U^{(2)}\right)$ назьвается полной, если $U^{(2)}=U^{(1)} \cap\left|A^{(2)}\right|$. Говорят, что $\left(U^{(1)}, U^{(2)}\right)$ - пара реперов в точке $\left(\alpha^{(1)}, \alpha^{(2)}\right) \in \Phi_{A^{(1)}} \times \Phi_{A^{(2)}}$, если $U^{(i)}-$ локальный репер в точке $\alpha^{(i)}$. Пара локальных реперов $\left(U^{(1)}, U^{(2)}\right)$ в точке $\left(\alpha^{(1)}, \alpha^{(2)}\right)$ называется относительны.м локальным.м репером в $\left(\alpha^{(1)}, \alpha^{(2)}\right)$ или $\left(\alpha^{(1)}, \alpha^{(2)}\right)$-относительным локальным репером, если $\left(G_{A^{(2)}}\right)_{\alpha^{(2)}}(u) \subseteq\left(G_{A^{(1)}}\right)_{\alpha^{(1)}}(u)$ для некоторого и, следовательно, для любого $u \in U^{(2)}$.

Чтобы определить относительную $A$-карту в $B$, где $A$ и $B$ - относительные действия, необходимо ввести глобальную структуру на множестве $\operatorname{Mor}(A, B)$ всех морфизмов из $A$ в $B$. Отметим, что если $\left|A^{(2)}\right|=\varnothing=\left|B^{(2)}\right|$, то понятие относительной карты будет совпадать с понятием карты для глобальных действий.

ОПРЕДЕЛЕНИЕ 4.8. Пусть $A$ и $B$ - относительные действия. Относительная $A$-карта, или просто $A$-карта, в $B$ состоит из морфизма относительных действий $f: A \rightarrow B$ и функции $\beta:\left|A^{(1)}\right| \rightarrow \Phi_{B^{(1)}} \cap \Phi_{B^{(2)}}$, для которых вьполняются следуюшие условия.

(4.8.0) Пусть $\left|A^{(1) \backslash(2)}\right|=\left|A^{(1)}\right| \backslash\left|A^{(2)}\right|$. Тогда функция $\beta$ переводит $\left|A^{(1) \backslash(2)}\right|$ в $\Phi_{B^{(1)}}$ и $\left|A^{(2)}\right|$ в $\Phi_{B^{(2)}}$.

(4.8.1) Если $x \in\left|A^{(1) \backslash(2)}\right|$, то $f(x) \in\left(X_{B^{(1)}}\right)_{\beta(x)}$. Если $x \in\left|A^{(2)}\right|$, то $f(x) \in$ $\left(X_{B^{(2)}}\right) \beta(x)$.

(4.8.2) Если $\left(U^{(1)}, U^{(2)}\right)$ - полная пара локальных реперов в $A$, то $\left(f U^{(1)}, f U^{(2)}\right)$ такой относительньй локальный репер в некоторой точке $\left(b^{(1)}, b^{(2)}\right) \in \Phi_{B^{(1)}} \times \Phi_{B^{(2)}}$, что $\beta(u) \leqslant b^{(1)}$ для всех $u \in U^{(1)} \backslash U^{(2)}$ и $\beta(u) \leqslant b^{(2)}$ для всех $u \in U^{(2)}$. 
ОПРЕДЕЛЕНИЕ-ЛЕМмА 4.9. Пусть $A$ и $B$ - относительные действия $u(f, \beta)$ - некоторая A-карта в $B$.

Для

$$
\sigma=\left(\sigma_{x}\right) \in \prod_{x \in\left|A^{(1) \backslash(2)}\right|}\left(G_{B^{(1)}}\right)_{\beta(x)} \times \prod_{x \in\left|A^{(2)}\right|}\left(G_{B^{(2)}}\right)_{\beta(x)}
$$

полоэи $и \mathcal{M}$

$$
\sigma f:\left|A^{(1)}\right| \rightarrow\left|B^{(1)}\right|, \quad x \mapsto \sigma_{x} f(x) .
$$

Тогда бf - морфизм относительных действий $A \rightarrow B u(\sigma f, \beta)$ - некоторая A-карта в $B$.

ДокАЗАтельство. Очевидно, что пара $(\sigma f, \beta)$ удовлетворяет условиям (4.8.0) и (4.8.1). Поэтому для доказательства леммы достаточно проверить условие (4.8.2). Пусть $\left(\left\{x_{0}, \ldots, x_{p}\right\},\left\{x_{0}, \ldots, x_{q}\right\}\right), q \leqslant p,-$ некоторая полная пара локальных реперов в $A$. По определению, $f\left(x_{0}\right), \ldots, f\left(x_{p}\right)$ является $b^{(1)}$-репером для некоторого $b^{(1)} \in \Phi_{B^{(1)}}, b^{(1)} \geqslant \beta\left(x_{q+1}\right), \ldots, \beta\left(x_{p}\right)$ a $f\left(x_{0}\right), \ldots, f\left(x_{q}\right)$ является $b^{(2)}$-репером для некоторого $b^{(2)} \in \Phi_{B^{(2)}}, b^{(2)} \geqslant \beta\left(x_{0}\right), \ldots, \beta\left(x_{q}\right)$, причем $\left(G_{B^{(2)}}\right)_{b^{(2)}} f\left(x_{0}\right) \subseteq$ $\left(G_{B^{(1)}}\right)_{b^{(1)}} f\left(x_{0}\right)$. Отсюда следует, что $\sigma_{x_{0}} f\left(x_{0}\right), \ldots, \sigma_{x_{p}} f\left(x_{p}\right)$ является $b^{(1)}$-репером, $\sigma_{x_{0}} f\left(x_{0}\right), \ldots, \sigma_{x_{q}} f\left(x_{q}\right)$ является $b^{(2)}$-репером и

$$
\left(G_{B^{(2)}}\right)_{b^{(2)}}\left(\sigma_{x_{0}} f\left(x_{0}\right)\right) \subseteq\left(G_{B^{(1)}}\right)_{b^{(1)}}\left(\sigma_{x_{0}} f\left(x_{0}\right)\right) .
$$

ОПРЕДЕЛЕНИЕ 4.10. Пусть $A$ и $B$ - относительные действия и $(f, \beta)$ - некоторая $A$-карта в $B$. (Относительным) $A$-репером в точке $f$ на карте $(f, \beta)$ назьвается такое множество морфизмов $f=f_{0}, f_{1}, \ldots, f_{p}: A \rightarrow B$, для которого сушествуют элементы

$$
\sigma_{1}, \ldots, \sigma_{p} \in \prod_{x \in\left|A^{(1) \backslash(2)}\right|}\left(G_{B^{(1)}}\right)_{\beta(x)} \times \prod_{x \in\left|A^{(2)}\right|}\left(G_{B^{(2)}}\right)_{\beta(x)},
$$

удовлетворяюшие соотношениям $\sigma_{i} f=f_{i}, 1 \leqslant i \leqslant p$. (В силу леммы 4.9 множество $f=f_{0}, f_{1}, \ldots, f_{p}$ является также $A$-репером в точке $f_{i}$ на карте $\left(f_{i}, \beta\right)$ для любого $i$ $(0 \leqslant i \leqslant p)$.)

Следующее полезное утверждение представляет собой аналог леммы 3.7.

ЛОКАЛЬНО-ГЛОБАЛЬНАЯ ЛЕММА 4.11. Пусть $A$ и $B$-относительные действия $u(f, \beta)$ - некоторая $A$-карта в $B$. Множество $f=f_{0}, f_{1}, \ldots, f_{p}$ является $A$-репером в точке $f$ на карте $(f, \beta)$ тогда и только тогда, когда для каждого $x \in\left|A^{(1)}\right|$ әлементы $f(x), f_{1}(x), \ldots, f_{p}(x)$ образуют локальный (не относительно локальный) репер в $f(x)$ для $\beta(x)$.

ДоКАЗАТЕЛЬСТВО является тривиальньм следствием леммы 4.9.

ОПРЕДЕЛЕНИЕ 4.12. Пусть $A, B$ и $C$ - относительные действия. Морфизм $g: B \rightarrow C$ называется $A$-нормальным морфизмом относительных действий, если $g: B^{(2)} \rightarrow C^{(2)}$ сохраняет $A^{(1)}$-реперы и $g: B \rightarrow C$ сохраняет $A$-реперы. (Напомним, что это означает следующее: если $f, f_{1}, \ldots, f_{p}$ - некоторый $A$-репер в точке $f$ на карте $(f, \beta)$, то $g f, g f_{1}, \ldots, g f_{p}$ является $A$-репером в точке $g f$ на некоторой $A$-карте $(g f, \gamma)$ в $C$.) Нормальнылм морфизмом относительных действий $g: B \rightarrow C$ назьвается морфизм, которьй сохраняет $A$-реперы для любого относительного действия $A$. Наконец, А-нормальный (соответственно, нормальный) изоморфизм - это такой 
$A$-нормальньй (соответственно, нормальный) морфизм, для которого существует $A$-нормальньй (соответственно, нормальный) обратньй.

В общем случае $A$-нормальный (соответственно, нормальный) морфизм, являюшийся изоморфизмом в обычном смысле, не обязан быть $A$-нормальным (соответственно, нормальным) изоморфизмом.

ЛЕмма 4.13. Любой регулярный морфизм относительных действий является нормальнылм морфизмом.

ДоКАЗАТЕЛЬСТВО. Пусть $\eta: B \rightarrow C$ - некоторьй регулярньй морфизм относительных действий. Если $(f, \beta)$ - произвольная $A$-карта в $B$, то прямая проверка показывает, что $\left(\eta_{X} f, \eta_{\Phi} \beta\right)$ является $A$-картой в $C$. Пусть $f, f_{1}, \ldots, f_{p}$ - какой либо $A$-репер в точке $f$ на карте $(f, \beta)$ и элементы

$$
\sigma_{1}, \ldots, \sigma_{p} \in \prod_{x \in\left|A^{(1) \backslash(2)}\right|}\left(G_{B^{(1)}}\right)_{\beta(x)} \times \prod_{x \in\left|A^{(2)}\right|}\left(G_{B^{(2)}}\right)_{\beta(x)}
$$

таковы, что $\sigma_{i} f=f_{i}, 1 \leqslant i \leqslant p$. Для

$$
\sigma=\left(\sigma_{x}\right) \in \prod_{x \in\left|A^{(1) \backslash(2)}\right|}\left(G_{B^{(1)}}\right)_{\beta(x)} \times \prod_{x \in\left|A^{(2)}\right|}\left(G_{B^{(2)}}\right)_{\beta(x)}
$$

положим

$$
\eta_{G}(\sigma)=\left(\eta_{G}(\beta(x))\left(\sigma_{x}\right)\right) \in \prod_{x \in\left|A^{(1) \backslash(2)}\right|}\left(G_{C^{(1)}}\right)_{\eta_{\Phi^{(1)}} \beta(x)} \times \prod_{x \in\left|A^{(2)}\right|}\left(G_{C^{(2)}}\right)_{\eta_{\Phi^{(2)}} \beta(x)} .
$$

Тогда согласно (3.3.4) имеет место соотношение $\eta_{G}\left(\sigma_{i}\right)\left(\eta_{X} f\right)=\eta_{X} f_{i}, 1 \leqslant i \leqslant p$. Следовательно, множество $\eta_{X} f, \eta_{X} f_{1}, \ldots, \eta_{X} f_{p}$ является $A$-репером в точке $\eta_{X} f$ на карте $\left(\eta_{X} f, \eta_{\Phi} \beta\right)$.

Зададим теперь структуру относительного действия на множестве $\operatorname{Mor}(A, B)$ всех морфизмов из относительного действия $A$ в относительное действие $B$.

ОПРЕДЕЛЕНИЕ 4.14. Пусть $A$ и $B$ - относительные действия. Определим относительное действие

$$
\operatorname{Mor}(A, B)=\left(\operatorname{Mor}(A, B)^{(1)}, \operatorname{Mor}(A, B)^{(2)}\right)
$$

следуюшим образом. Прежде всего положим

$$
\operatorname{Mor}(A, B)^{(2)}=\operatorname{Mor}\left(A^{(1)}, B^{(2)}\right) .
$$

Зададим $\operatorname{Mor}(A, B)^{(1)}$ как глобальное действие с обертьваюшим множеством $|\operatorname{Mor}(A, B)|$ и глобальной структурой

$$
\left(\Phi_{(A, B)^{(1)}}, G_{(A, B)^{(1)}}, X_{(A, B)^{(1)}}\right),
$$

определяемой следующими правилами.

$$
\Phi_{(A, B)^{(1)}}=\left\{\beta:|A| \rightarrow \Phi_{B^{(1)}} \cup \Phi_{B^{(2)}} \mid(4.8 .0) \text { удовлетворены }\right\} .
$$

Наделим множество $\Phi_{(A, B)^{(1)}}$ транзитивным рефлексивным отношением, полагая $\beta \leqslant \beta^{\prime} \Leftrightarrow \beta(x) \leqslant \beta^{\prime}(x) \forall x \in\left|A^{(1)}\right|$. Для $\beta \in \Phi_{(A, B)^{(1)}}$, определим $\left(G_{(A, B)^{(1)}}\right)_{\beta}$ как

$$
\left(G_{(A, B)^{(1)}}\right)_{\beta}=\prod_{x \in\left|A^{(1) \backslash(2)}\right|}\left(G_{B^{(1)}}\right)_{\beta(x)} \times \prod_{x \in\left|A^{(2)}\right|}\left(G_{B^{(2)}}\right)_{\beta(x)} .
$$


Если $\beta \leqslant \beta^{\prime}$, то для каждого $x \in\left|A^{(1) \backslash(2)}\right|$ существует функториально определенный гомоморфизм $\left(G_{B^{(1)}}\right)_{\beta(x)} \rightarrow\left(G_{B^{(1)}}\right)_{\beta^{\prime}(x)}$ и для каждого $x \in\left|A^{(2)}\right|-$ функториально определенньй гомоморфизм $\left(G_{B^{(2)}}\right)_{\beta(x)} \rightarrow\left(G_{B^{(2)}}\right)_{\beta^{\prime}(x)}$. Следовательно, определен гомоморфизм $\left(G_{(A, B)}\right)_{\beta} \longrightarrow\left(G_{(A, B)}\right)_{\beta^{\prime}}$, функториально зависящий от $\beta$. Для $\beta \in \Phi_{(A, B)}(1)$ положим

$$
\left(X_{(A, B)^{(1)}}\right)_{\beta}=\left\{f:\left|A^{(1)}\right| \rightarrow\left|B^{(1)}\right| \mid(f, \beta) \text { есть } A \text {-карта в } B\right\} .
$$

Если $\sigma \in\left(G_{(A, B)^{(1)}}\right)_{\beta}$ и $f \in\left(X_{(A, B)^{(1)}}\right)_{\beta}$, то из леммы 4.9 следует, что $\sigma f \in$ $\left(X_{(A, B)^{(1)}}\right)_{\beta}$. Это означает, что на $\left(X_{(A, B)^{(1)}}\right)_{\beta}$ задано действие группы $\left(G_{(A, B)^{(1)}}\right)_{\beta}$. Легко проверяется, что пара $\left(\operatorname{Mor}(A, B)^{(1)}, \operatorname{Mor}(A, B)^{(2)}\right)$ удовлетворяет всем условиям для относительного действия.

ПРЕДЛОЖЕНИЕ 4.15. Как функтор, принимающий значения в относительных действиях, Mor( , ) контравариантен и регулярен относительно всех морфизмов по первому аргументу при условии, что для второго аргумента допускаются только регулярнье относительные действия, и ковариантен относительно всех нормальных морфизмов по второму аргументу. Более подробно, имеют место следующие утверждения.

(4.15.1) Пусть $C$ - регулярное относительное действие и $f: A \rightarrow B-$ морфизм относительных действий. Тогда $f$ следующим образом определяет регулярный морфизм

$$
\eta=\operatorname{Mor}\left(f, 1_{C}\right): \operatorname{Mor}(B, C) \rightarrow \operatorname{Mor}(A, C) .
$$

Обозначим через $\nu: \Phi_{C^{(1)}} \rightarrow \Phi_{C^{(2)}}$ каноническое отображение и зададим сохраняющий отношения морфизм

$$
\eta_{\Phi^{(1)}}: \Phi_{(B, C)^{(1)}} \rightarrow \Phi_{(A, C)^{(1)}},
$$

полагая

$$
\eta_{\Phi^{(1)}}(\beta)=\beta \circ f
$$

2дe

$$
\beta \circ f(x)= \begin{cases}\beta(f(x)), & \text { если } x \in\left|A^{(1) \backslash(2)}\right| u f(x) \notin B^{(2)}, \\ \nu \beta(f(x)), & \text { если } x \in\left|A^{(1) \backslash(2)}\right| u f(x) \in B^{(2)}, \\ \beta(f(x)), & \text { если } x \in\left|A^{(2)}\right| .\end{cases}
$$

Определим естественное преобразование

$$
\eta_{G^{(1)}}: G_{(B, C)^{(1)}} \rightarrow G_{(A, C)^{(1)}}
$$

как семейство

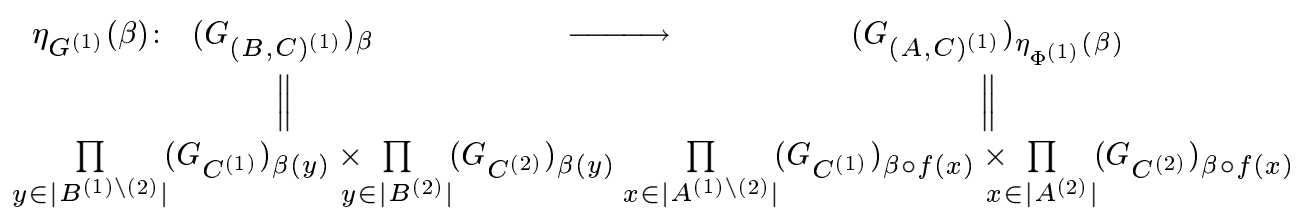

әде

$$
\left.\eta_{G^{(1)}}(\beta)\right|_{\left(G_{C^{(i)}}\right)_{\beta(y)}}
$$


$\left(i=1\right.$, если $y \notin\left|B^{(2)}\right|$, и $i=2$, если $\left.y \in\left|B^{(2)}\right|\right)$ - диагональньй гомоморфизм

$$
\left(G_{C^{(2)}}\right)_{\beta(y)} \longrightarrow \prod_{x \in\left|A^{(1)}\right|, f(x)=y}\left(G_{C^{(i)}}\right)_{\beta \circ f(x)}
$$

Прямая проверка показывает, что тройка

$$
\eta^{(1)}=\left(\eta_{\Phi^{(1)}}, \eta_{G^{(1)}}, \eta_{X}\right)
$$

определяет регулярный морфизм глобальных действий

$$
\eta^{(1)}: \operatorname{Mor}(B, C)^{(1)} \rightarrow \operatorname{Mor}(A, C)^{(1)}
$$

и что ограничение $\eta^{(2)}=\left.\eta_{X}\right|_{\operatorname{Mor}(B, C)^{(2)}}$ задает морфизм глобальньх действий

$$
\eta^{(2)}: \operatorname{Mor}(B, C)^{(2)} \rightarrow \operatorname{Mor}(A, C)^{(2)}
$$

Используя (3.11.1), наделим $\eta^{(2)}$ регулярной структурой. Тогда пара

$$
\eta=\left(\eta^{(1)}, \eta^{(2)}\right)
$$

является регулярным морфизмом относительных действий

$$
\eta: \operatorname{Mor}(B, C) \rightarrow \operatorname{Mor}(A, C) .
$$

Кроме того, $\operatorname{Mor}(B, C)$ и $\operatorname{Mor}(A, C)$ являются регулярными относительными действиями, регулярные структурь на которых индуиировань регулярной структурой на $C$.

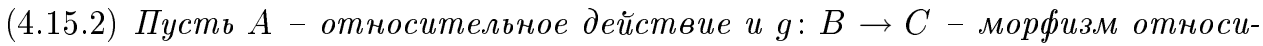
тельных действий. Функиия

$$
\operatorname{Mor}\left(1_{A}, g\right):\left|\operatorname{Mor}(A, B)^{(1)}\right| \rightarrow\left|\operatorname{Mor}(A, C)^{(1)}\right|
$$

задает морфизм глобальньх действий $\operatorname{Mor}(A, B) \rightarrow \operatorname{Mor}(A, C)$ тогда и только тогда, когда $g$ является $A$-нормальным.м.

ДокАЗАТЕльство. (4.15.1) В этом пункте все доказано. (4.15.2) Пусть $(f, \beta)-$ некоторая $A$-карта в $B,\left(f^{\prime}, \beta^{\prime}\right)$ - некоторая $A^{(1)}$-карта в $B^{(2)}, f=f_{0}, f_{1}, \ldots, f_{p}$ - какой-либо $A$-репер на $(f, \beta)$ и $f^{\prime}=f_{0}^{\prime}, f_{1}^{\prime}, \ldots, f_{p}^{\prime}$ - какой-либо $A^{(1)}$-репер на $\left(f^{\prime}, \beta^{\prime}\right)$. Из определения следует, что $f_{0}, \ldots, f_{p}$ является также локальным $\beta$-репером в глобальном действии $\operatorname{Mor}(A, B)^{(1)}$; обратно, любой локальный репер в $\operatorname{Mor}(A, B)^{(1)}$ есть в то же время $A$-репер на некоторой $A$-карте в $B$. Аналогично, $f_{0}^{\prime}, \ldots, f_{p^{\prime}}^{\prime}-$ локальньй $\beta^{\prime}$-репер в глобальном действии $\operatorname{Mor}(A, B)^{(2)}$, и любой локальньй репер в $\operatorname{Mor}(A, B)^{(2)}$ является $A^{(1)}$-репером на некоторой $A^{(1)}$-картев $B^{(2)}$. Следовательно, функция $\operatorname{Mor}\left(1_{A}, g\right):\left|\operatorname{Mor}(A, B)^{(1)}\right| \rightarrow\left|\operatorname{Mor}(A, C)^{(1)}\right|$ определяет морфизм относительных действий $\operatorname{Mor}(A, B) \rightarrow \operatorname{Mor}(A, C)$ тогда и только тогда, когда $\operatorname{Mor}\left(1_{A}, g\right)$ сохраняет $A$-реперы, а $\operatorname{Mor}\left(1_{A}, g\right)^{(2)}:\left|\operatorname{Mor}(A, B)^{(2)}\right| \rightarrow\left|\operatorname{Mor}(A, C)^{(2)}\right|$ сохраняет $A^{(1)}$-реперы. В свою очередь, это условие вьполняется тогда и только тогда, когда морфизм $g$ является $A$-нормальным. 
ОПРЕДЕЛЕНИЕ 4.16. Пусть $g: B \rightarrow C$ - морфизм относительных действий. Последовательность $A_{n}, \ldots, A_{1}$ относительных действий назьвается нормальной це$n ь ю$ длины $n$ для $g$, если морфизм $g$ является $A_{1}$-нормальным и если для любого $i$, $0 \leqslant i \leqslant n-1$, морфизм

$$
\begin{aligned}
& \left.\operatorname{Mor}\left(1_{A_{i-1}}, \ldots, \operatorname{Mor}\left(1_{A_{1}}, g\right)\right) \ldots\right): \\
& \quad \operatorname{Mor}\left(A_{i}, \operatorname{Mor}\left(A_{i-1}, \ldots, \operatorname{Mor}\left(A_{1}, B\right)\right) \ldots\right) \rightarrow \operatorname{Mor}\left(A_{i}, \operatorname{Mor}\left(A_{i-1}, \ldots, \operatorname{Mor}\left(A_{1}, C\right)\right) \ldots\right)
\end{aligned}
$$
является $A_{i+1}$-нормальным. Пусть $\mathscr{N}$ - некоторый класс относительных действий. Морфизм $g$ называется $n$ - $\mathscr{N}$-нормальным, если каждая последовательность из $n$ объектов, принадлежащих $\mathscr{N}$, образует нормальную цепь для $g$. Морфизм $g$ назьвается $\mathscr{N}$-нормальным (соответственно, $\infty$ - $\mathscr{N}$-нормальнылм), если он 1- $\mathscr{N}$-нормален (соответственно, $n$ - $\mathscr{N}$-нормален для всех $n>0)$. Если $\mathscr{N}=\{A\}($ соответственно, $\mathscr{N}-$ класс всех относительных действий), мы будем назьвать соответствующий морфизм $\infty$ - $A$-нормальным (соответственно, $\infty$-нормальным $)$.

Если термином $t$-морфизм обозначить один из определенных вьше вариантов нормального или регулярного морфизма, то $t$-изоморфизмом назьвается $t$-морфизм, обладаюший обратньм $t$-морфизмом.

Лемма 4.17. Пусть $g: B \rightarrow C-$ регулярный морфизм относительных действий. Тогда для любого относительного действия $A$ морфизм $\operatorname{Mor}\left(1_{A}, g\right)$ : $\operatorname{Mor}(A, B) \rightarrow \operatorname{Mor}(A, C)$ регулярен $u$, следовательно, g является $\infty$-нормальньм морфизмом.

ДОКАЗАТЕЛЬСТВО. Согласно лемме 4.13 и предложению 4.15.2 морфизм

$$
\operatorname{Mor}\left(1_{A}, g\right): \operatorname{Mor}(A, B) \rightarrow \operatorname{Mor}(A, C)
$$

определен корректно. Пусть $\left(\eta_{\Phi}, \eta_{G}, \eta_{X}=g\right)$ - регулярная структура морфизма $g$. Зададим регулярную структуру $\left(\mu_{\Phi}, \mu_{g}, \mu_{X}=\operatorname{Mor}\left(1_{A}, g\right)\right)$ для морфизма Mor $\left(1_{A}, g\right)$.

Определим, прежде всего, координатные морфизмы, полагая

$$
\begin{array}{ll}
\mu_{\Phi^{(1)}}: \Phi_{(A, B)^{(1)}} \rightarrow \Phi_{(A, C)^{(1)}}, & \beta \mapsto \eta_{\Phi^{(1)}} \beta, \\
\mu_{\Phi^{(2)}}: \Phi_{(A, B)^{(2)}} \rightarrow \Phi_{(A, C)^{(2)}}, & \beta \mapsto \eta_{\Phi^{(2)}} \beta .
\end{array}
$$

Далее, определим естественное преобразование

$$
\mu_{G^{(1)}}: G_{(A, B)^{(1)}} \rightarrow G_{(A, C)^{(1)}}
$$

с помощью коммутативной диаграммы

где

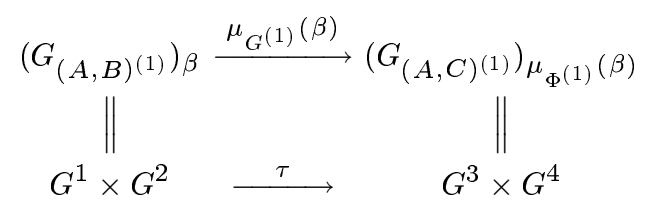

$$
\begin{gathered}
\tau=\prod_{x \in\left|A^{(1) \backslash(2)}\right|} \eta_{G^{(1)}}(\beta(x)) \times \prod_{x \in\left|A^{(2)}\right|} \eta_{G^{(1)}}(\beta(x)), \\
G^{1}=\prod_{x \in\left|A^{(1) \backslash(2)}\right|}\left(G_{B^{(1)}}\right)_{\beta(x)}, \quad G^{2}=\prod_{x \in\left|A^{(2)}\right|}\left(G_{B^{(2)}}\right)_{\beta(x)}, \\
G^{3}=\prod_{x \in\left|A^{(1) \backslash(2)}\right|}\left(G_{C^{(1)}}\right)_{\eta_{\Phi^{(1)}}(\beta(x))}, \quad G^{4}=\prod_{x \in\left|A^{(2)}\right|}\left(G_{\left.C^{(2)}\right)} \eta_{\Phi^{(2)}}(\beta(x)) .\right.
\end{gathered}
$$


Кроме того, зададим естественное преобразование

$$
\mu_{G^{(2)}}: G_{(A, B)^{(2)}} \rightarrow G_{(A, C)^{(2)}}
$$

так же, как в доказательстве леммы 3.13.

Прямая проверка показьвает, что $\left(\mu_{\Phi}, \mu_{G}, \operatorname{Mor}\left(1_{A}, g\right)\right)$ является регулярным морфизмом. Тот факт, что морфизм $g$ является $\infty$-нормальным, получается отсюда элементарной индукцией.

ОПРЕДЕЛЕНИЕ 4.18. Пусть $N$ - название одного из типов морфизмов, определенных в 4.16. Относительное действие назьвается $N$-действием, если каждый его морфизм является $N$-морфизмом.

ОПРЕДЕЛЕНИЕ 4.19. Пусть $A$ и $B$ - относительные действия. Определим относительное действие

$$
A \bowtie B
$$

следуюшим образом:

$$
(A \bowtie B)^{(1)}=A^{(1)} \times B^{(1)} .
$$

Обертьвающее множество для $(A \bowtie B)^{(2)}$ задается формулой

$$
\left|(A \bowtie B)^{(2)}\right|=\left|A^{(1)}\right| \times\left|B^{(2)}\right| \cup\left|A^{(2)}\right| \times\left|B^{(1)}\right|,
$$

где объединение берется внутри множества $\left|A^{(1)}\right| \times\left|B^{(1)}\right|$. Координатная система для $(A \bowtie B)^{(2)}$ есть

$$
\Phi_{(A \bowtie B)^{(2)}}=\Phi_{A^{(1)}} \times \Phi_{B^{(2)}} \cup \Phi_{A^{(2)}} \times \Phi_{B^{(1)}},
$$

где на сей раз рассматривается несвязное объединение. Морфизмы между элементами определяются покоординатно. Глобальньй групповой функтор для $(A \bowtie B)^{(2)}$ определяется правилом

$$
\left(G_{(A \bowtie B)^{(2)}}\right)_{(\alpha, \beta)}= \begin{cases}\left(G_{A^{(1)}}\right)_{\alpha} \times\left(G_{B^{(2)}}\right)_{\beta} & \text { если }(\alpha, \beta) \in \Phi_{A^{(1)}} \times \Phi_{B^{(2)}}, \\ \left(G_{A^{(2)}}\right)_{\alpha} \times\left(G_{B^{(1)}}\right)_{\beta} & \text { если }(\alpha, \beta) \in \Phi_{A^{(2)}} \times \Phi_{B^{(1)}} .\end{cases}
$$

Глобальная функция множества для $(A \bowtie B)^{(2)}$ определяется правилом

$$
\left(X_{(A \bowtie B)^{(2)}}\right)_{(\alpha, \beta)}= \begin{cases}\left(X_{A^{(1)}}\right)_{\alpha} \times\left(X_{B^{(2)}}\right)_{\beta} & \text { если }(\alpha, \beta) \in \Phi_{A^{(1)}} \times \Phi_{B^{(2)}}, \\ \left(X_{A^{(2)}}\right)_{\alpha} \times\left(X_{B^{(1)}}\right)_{\beta} & \text { если }(\alpha, \beta) \in \Phi_{A^{(2)}} \times \Phi_{B^{(1)}} .\end{cases}
$$

Группа $\left(G_{(A \bowtie B)^{(2)}}\right)_{(\alpha, \beta)}$ действует на множестве $\left(X_{(A \bowtie B)^{(2)}}\right)_{(\alpha, \beta)}$ покоординатно.

Далее мы будем использовать обозначение $(S, T)=\operatorname{Mor}_{\text {sets }}(S, T)$, где $S$ и $T$ - некоторые множества. Это обозначение было введено в определении 3.16.

ОПРЕДЕЛЕНИЕ 4.20. Пусть $A, B$ и $C$-относительные действия. Регулярньй морфизм

$$
E: \operatorname{Mor}(A, \operatorname{Mor}(B, C)) \rightarrow \operatorname{Mor}(A \bowtie B, C)
$$

определяется следуюшим образом. Обозначим структурные компоненты относительного действия $\operatorname{Mor}(A, \operatorname{Mor}(B, C))$ через $\left(\Phi_{(A,(B, C))}, G_{(A,(B, C))}, X_{(A,(B, C))}\right)$. Определим $E_{\Phi^{(1)}}$ так, чтобы имела место коммутативная диаграмма

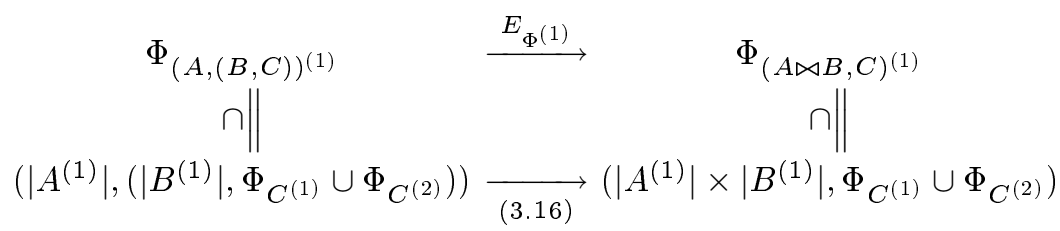


Ясно, что $E_{\Phi^{(1)}}$ сохраняет транзитивное реф̆лексивное отношение. Определим $E_{\Phi^{(2)}}$ так же, как в (3.17), т.е. так, чтобы имела место коммутативная диаграмма

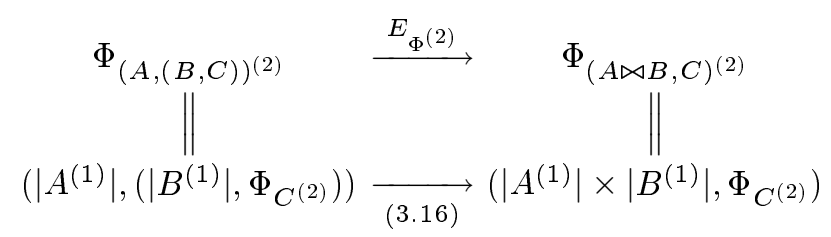

Зададим естественное преобразование

$$
E_{G^{(1)}}: G_{(A,(B, C))^{(1)}} \rightarrow\left(G_{(A \bowtie B, C)^{(1)}}\right)_{E_{\Phi^{(1)}}()}
$$

так, чтобы гомоморфизм

$$
E_{G^{(1)}}(\alpha):\left(G_{(A,(B, C))^{(1)}}\right)_{\alpha} \longrightarrow\left(G_{(A \bowtie B, C)^{(1)}}\right)_{E_{\Phi^{(1)}}(\alpha)}
$$

тождественно отображал сомножитель из

$$
\begin{aligned}
\left(G_{\left.(A,(B, C))^{(1)}\right)_{\alpha}=}\right. & \prod_{x \in\left|A^{(1) \backslash(2)}\right|}\left[\left(\prod_{y \in\left|B^{(1) \backslash(2)}\right|}\left(G_{C^{(1)}}\right)_{\alpha(x)(y)}\right) \times\left(\prod_{y \in\left|B^{(2)}\right|}\left(G_{C^{(2)}}\right)_{\alpha(x)(y)}\right)\right] \\
& \times \prod_{x \in\left|A^{(2)}\right|}\left[\prod_{y \in\left|B^{(1)}\right|}\left(G_{C^{(2)}}\right)_{\alpha(x)(y)}\right]
\end{aligned}
$$

с индексом $\alpha(x)(y)$ на сомножитель из

$$
\begin{aligned}
\left(G_{(A \bowtie B, C)^{(1)}}\right)_{\alpha}= & \prod_{(x, y) \in\left|A^{(1) \backslash(2)}\right| \times\left|B^{(1) \backslash(2)}\right|}\left(G_{C^{(1)}}\right)_{\left(E_{\Phi^{(1)}} \alpha\right)(x, y)} \\
& \times \prod_{(x, y) \in\left|(A \bowtie B)^{(2)}\right|}\left(G_{C^{(2)}}\right)_{\left(E_{\Phi^{(1)}} \alpha\right)(x, y)}
\end{aligned}
$$

с индексом $\left(E_{\Phi^{(1)}} \alpha\right)(x, y)$. Определим естественное преобразование

$$
E_{G^{(2)}}: G_{(A,(B, C))^{(2)}} \rightarrow G_{(A \bowtie B, C)^{(2)}}
$$

так же, как в определении 3.17 , т.е. так, чтобы гомоморфизм

$$
E_{G^{(2)}}(\alpha):\left(G_{(A,(B, C))^{(2)}}\right)_{\alpha} \rightarrow\left(G_{(A \bowtie B, C)^{(2)}}\right)_{E_{\Phi^{(2)}}(\alpha)}
$$

тождественно отображал сомножитель из

$$
\left(G_{(A,(B, C))^{(2)}}\right)_{\alpha}=\prod_{x \in\left|A^{(1)}\right|}\left(\prod_{y \in\left|B^{(1)}\right|}\left(G_{C^{(2)}}\right)_{\alpha(x)(y)}\right)
$$

с индексом $\alpha(x)(y)$ на сомножитель из

$$
\left(G_{(A \bowtie B, C)^{(2)}}\right)_{E_{\Phi^{(2)}}(\alpha)}=\prod_{(x, y) \in\left|A^{(1)}\right| \times\left|B^{(1)}\right|}\left(G_{C^{(2)}}\right)_{\left(E_{\Phi^{(2)}} \alpha\right)(x, y)}
$$


с индексом $\left(E_{\Phi^{(2)}} \alpha\right)(x, y)$. Прямая проверка показывает, что образ композиции отображений

$$
\left|\operatorname{Mor}(A, \operatorname{Mor}(B, C))^{(1)}\right| \longrightarrow\left(\left|A^{(1)}\right|,\left(\left|B^{(1)}\right|,\left|C^{(1)}\right|\right)\right) \stackrel{(3.16)}{\longrightarrow}\left(\left|A^{(1)}\right| \times\left|B^{(1)}\right|, C^{(1)} \mid\right)
$$

лежит в $\left|\operatorname{Mor}(A \bowtie B, C)^{(1)}\right|$. Поэтому определим

$$
E_{X}:\left|\operatorname{Mor}(A, \operatorname{Mor}(B, C))^{(1)}\right| \rightarrow\left|\operatorname{Mor}(A \bowtie B, C)^{(1)}\right|
$$

как результирующее отображение. Легко видеть, что тройка

$$
E^{(1)}=\left(E_{\Phi^{(1)}}, E_{G^{(1)}}, E_{X}\right)
$$

является регулярным морфизмом глобальных действий

$$
E^{(1)}: \operatorname{Mor}(A, \operatorname{Mor}(B, C))^{(1)} \rightarrow \operatorname{Mor}(A \bowtie B, C)^{(1)} .
$$

В обшем случае $E^{(1)}$ не является изоморфизмом (соответственно, регулярньм изоморфизмом), поскольку отображение $E_{X}$ не обязано быть сюръективным (соответственно, $E_{X}\left(\left(X_{(A,(B, C))^{(1)}}\right)_{\alpha}\right)$ не обязано совпадать с $\left.\left(X_{(A \bowtie B, C)^{(1)}}\right)_{E_{\Phi^{(1)}}(\alpha)}\right)$. Пусть

и

$$
E_{X^{(2)}}=\left.E_{X}\right|_{\left|\operatorname{Mor}(A,(B, C))^{(2)}\right|}
$$

Тогда в силу 3.17

$$
E^{(2)}=\left(E_{\Phi^{(2)}}, E_{G^{(2)}}, E_{X^{(2)}}\right) .
$$

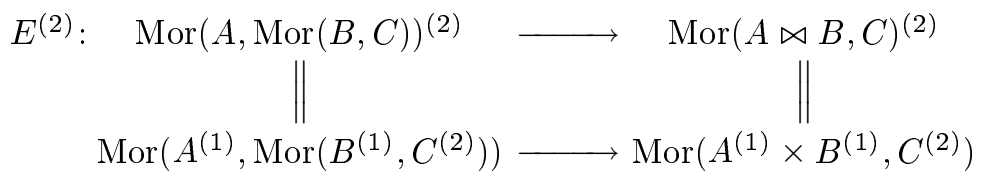

является регулярньм морфизмом глобальных действий. Непосредственно проверяется, что пара

$$
E=\left(E^{(1)}, E^{(2)}\right)
$$

представляет собой регулярньй морфизм

$$
E: \operatorname{Mor}(A, \operatorname{Mor}(B, C)) \rightarrow \operatorname{Mor}(A \bowtie B, C)
$$

относительных действий.

Пусть $A_{n}, \ldots, A_{1}$ - произвольная последовательность глобальных действий. Итерируя описанную выше процедуру, определим для любого $n \geqslant 2$ регулярный морфизм

$$
E_{n}: \operatorname{Mor}\left(A_{n}, \operatorname{Mor}\left(A_{n-1}, \ldots, \operatorname{Mor}\left(A_{1}, C\right)\right) \ldots\right) \rightarrow \operatorname{Mor}\left(A_{n} \bowtie \cdots \bowtie A_{1}, C\right)
$$

следуюшим образом. Для $n=2$ морфизм был определен выше. Предположим поэтому, что $n>2$ и что морфизм уже определен для любого натурального числа $N$, где $2 \leqslant N \leqslant n-1$. Пусть $E_{n-1}-$ соответствующий морфизм для последовательности $A_{n-1}, \ldots, A_{1}$. Определим морфизм $E_{n}$ для последовательности $A_{n}, A_{n-1}, \ldots, A_{1}$ как композицию регулярного морфизма $\operatorname{Mor}\left(1_{A_{n}}, E_{n-1}\right)($ см. $(4.17))$ и регулярного морфизма

$$
E_{2}: \operatorname{Mor}\left(A_{n}, \operatorname{Mor}\left(A_{n-1} \bowtie \cdots \bowtie A_{1}, B\right)\right) \rightarrow \operatorname{Mor}\left(A_{n} \bowtie \cdots \bowtie A_{1}, B\right) .
$$

Займемся теперь проблемой нахождения обратного морфизма для $E_{n}$. 
ОПРЕДЕЛЕНИЕ 4.21. Пусть $\mathscr{P}$ - некоторьй класс относительных действий, замкнутьй относительно конечного числа операций $\bowtie$. Относительное действие $C$ называется $\infty$ - $\mathscr{P}$-әкспоненииальнылм, если морфизм $E$ является $\infty$ - $\mathscr{P}$-нормальным морфизмом для всех пар $A, B \in \mathscr{P}$. Действие $C$ назьвается регулярно- $\infty$ - $\mathscr{P}$-әкспоненuиальнымм, если $E$ регулярный изоморфизм для всех пар $A, B \in \mathscr{P}$. Если класс $\mathscr{P}$ состоит из всех конечных $\bowtie$-произведений некоторого относительного действия $A$ (соответственно, из всех таких относительных действий $A$, что $\left|A^{(i)}\right|=\bigcup_{\alpha \in \Phi_{A^{(i)}}}\left(X_{A^{(i)}}\right)_{\alpha}$, $i=1,2)$, то $\infty$ - $\mathscr{P}$-экспоненциальное действие $C$ назьвается $\infty-A$-әкспоненциальнылм (соответственно, $\infty$-әкспоненииальным.м.

Лемма 4.22. Предположил, что относительное действие $C$ является $\infty$-Я әкспоненциальным (соответственно, регулярно $\infty$-Я̈-экспоненциальньл). Тогда для любой последовательности $A_{n}, \ldots, A_{1} \in \mathscr{P}, n \geqslant 2$, морфизм $E_{n}$ из определения 4.20 является $\infty$-Я -нормальным (соответственно, регулярным) изоморфизмом.

ДоКАЗАТЕЛЬСТВо в точности совпадает с доказательством леммы 3.19.

ОПРЕДЕЛЕНИЕ 4.23. Пусть $A$ и $B$ - относительные действия. Морфизм $f: A \rightarrow B$ называется четким, если он сохраняет относительные локальные реперы. Более подробно, если $U^{(i)} \subseteq\left|A^{(i)}\right|(i=1,2)$ - такие непустые подмножества, что $U^{(2)} \subseteq U^{(1)}$ и пара $\left(U^{(1)}, U^{(2)}\right)$ образует относительный локальньй репер в $A$, то пара $\left(f U^{(1)}, f U^{(2)}\right)$ образует относительньй локальный репер в $B$.

ОПрЕДЕЛЕНИЕ 4.24. Относительное действие $A$ назьвается сильньм четким действием, если выполняется следующее условие.

(4.24.1) Пусть $\Delta^{(2)} \subseteq \Phi_{A^{(2)}}-$ произвольное конечное множество и $U^{(i)} \subseteq\left|A^{(i)}\right|$ $(i=1,2)$ - такие непустые конечные подмножества, что $U^{(2)} \subseteq U^{(1)}$. Предположим, что для каждого $\delta^{(2)} \in \Delta^{(2)}$ пересечение $\left(X_{A^{(2)}}\right)_{\delta^{(2)}} \cap U^{(2)}$ не пусто. Кроме того, предположим, что $U^{(i)}$ - такой локальньй репер с точке $\alpha^{(i)} \in \Phi_{A^{(i)}}(i=1,2)$, что $\left(G_{A^{(2)}}\right)_{\delta^{(2)}}(u) \subseteq\left(G_{A^{(1)}}\right)_{\alpha^{(1)}}(u)$ для всех $\delta^{(2)} \in \Delta^{(2)}, \delta^{(2)} \leqslant \alpha^{(2)}, u \in\left(X_{A^{(2)}}\right)_{\delta^{(2)}} \cap U^{(2)}$. Тогда существует такой элемент $\beta^{(2)} \in \Phi_{A^{(2)}}$, что $\delta^{(2)} \leqslant \beta^{(2)} \leqslant \alpha^{(2)}$ для всех $\delta^{(2)} \in$ $\Delta^{(2)}$ и $\left(U^{(1)}, U^{(2)}\right)$ является относительным локальным репером в точке $\left(\alpha^{(1)}, \beta^{(2)}\right)$.

Относительное действие $A$ назьвается четким действием, если условие (4.24.1) вьполняется по меншшей мере для $\Delta^{(2)}=\varnothing$.

Подпространство $B \subseteq A$ глобального действия $A$ назьвается четким (соответственно, сильным четким) подпространством, если пара $(A, B)$ представляет собой четкое (соответственно, сильное четкое) относительное действие.

Ясно, что пунктированное действие является сильным четким относительньм действием.

Лемма 4.25. Пусть $f: A \rightarrow B$ - морфизм относительньх действий, причем $B$ является четким. Тогда $f-$ четкий морфизм.

ДокАЗАтЕЛьство. Рассмотрим некоторый относительный репер $\left(U^{(1)}, U^{(2)}\right)$ в $A$. Так как $f$ - морфизм относительных действий, то пара $\left(f U^{(1)}, f U^{(2)}\right)$ удовлетворяет условию (4.24.1) для $\Delta^{(2)}=\varnothing$. Следовательно, $\left(f U^{(1)}, f U^{(2)}\right)$ - относительный локальный репер в $B$.

Следующее условие дает нам полезньй критерий, гарантируюший, что относительное действие является либо $\infty$-нормальньм, либо $\infty$-экспоненциальным, либо регулярно $\infty$-экспоненциальным. 
ОПРЕДЕЛЕНИЕ 4.26. Относительное действие $A$ назьвается действием $c$ сильной нижней гранью, если $A^{(1)}$ и $A^{(2)}$ - действия с сильной нижней гранью и вьполнено следуюшее условие.

(4.26.1) Пусть $\Delta^{(i)} \subseteq \Phi_{A^{(i)}}(i=1,2)$ - произвольные конечные подмножества и $U^{(i)} \subseteq\left|A^{(i)}\right|(i=1,2)$ - такие непустые конечные подмножества, что $U^{(2)} \subseteq U^{(1)}$. Предположим, что для каждого $\delta^{(i)} \in \Delta^{(i)}$ пересечение $\left(X_{A^{(i)}}\right)_{\delta^{(i)}} \cap U^{(i)}$ не пусто. Кроме того, предположим, что не пусты множества $\Psi^{(1)}=\left\{\alpha^{(1)} \in \Phi_{A^{(1)}}^{\geqslant \Delta^{(1)}} \mid U^{(1)}\right.$ есть $\alpha^{(1)}$-репер, $\delta^{(1)} \leqslant \alpha^{(1)} \forall \delta^{(1)} \in \Delta^{(1)},\left(G_{A^{(2)}}\right)_{\delta^{(2)}}(u) \subseteq\left(G_{A^{(1)}}\right)_{\alpha^{(1)}}(u)^{A} \forall \delta^{(2)} \in \Delta^{(2)}$ и $\left.u \in\left(X_{A^{(2)}}\right)_{\delta^{(2)}} \cap U^{(2)}\right\}$ и $\Psi^{(2)}=\left\{\alpha^{(2)} \in \Phi_{A^{(2)}} \mid U^{(2)}\right.$ есть $\alpha^{(2)}$-репер, $\delta^{(2)} \leqslant \alpha^{(2)}$ $\left.\forall \delta^{(2)} \in \Delta^{(2)}\right\}$. Тогда множества $\Psi^{(i)}(i=1,2)$ содержат такой инициальньй элемент $\beta^{(i)}$, что $\left(G_{A^{(2)}}\right)_{\beta^{(2)}}(u) \subseteq\left(G_{A^{(1)}}\right)_{\beta^{(1)}}(u)$ для $u \in U^{(2)}$.

Относительное действие $A$ назьвается действием с нижней гранью, если $A^{(1)}$ и $A^{(2)}$ - действия с нижней гранью и условие (4.26.1) выполнено по меньшей мере для пары множеств $\left(\Delta^{(1)}, \Delta^{(2)}\right)=(\varnothing, \varnothing)$.

Легко видеть, что имеет место следующее утверждение.

ЛЕмма 4.27. Стандартное пунктированное действие $A$ является относительным действием с нижней гранью (соответственно, сильной нижней гранью) тогда и только тогда, когда $A^{(1)}$ - глобальное действие с нижней гранью (соответственно, сильной нижней гранью).

Лемма 4.28. Относительное действие $A$ обладает нижней гранью (соответственно, сильной нижней гранью) тогда и только тогда, когда оно является четким, (соответственно, сильным четким) и $A^{(i)}(i=1,2)$ - глобальное действие с нижней гранью (соответственно, сильной нижней гранью).

ДокАЗАТЕльство. $\Rightarrow$ ) Предположим, что выполнены предположения, фигурирующие в условии (4.24.1). Мы должны показать, что тогда справедливо заключение этого условия, необходимое для четких или сильно четких действий. Пусть $\Delta^{(1)}=\varnothing$ и $\Psi^{(i)}(i=1,2)$ - множества, определенные в (4.26.1). Так как элементы $\alpha^{(i)}$, описанные в определении $(4.24 .1)$, лежат в множествах $\Psi^{(i)}(i=1,2)$, то эти множества непусты. Пусть $\beta^{(i)} \in \Psi^{(i)}$ - инициальные элементы множеств $\Psi^{(i)}$, существование которых гарантируется условием (4.26.1). Из определения инициальности следует, что $\beta^{(i)} \leqslant \alpha^{(i)}$. Кроме того, заключение условия (4.26.1) гласит, что $U^{(2)}$ является $\beta^{(2)}$-репером, $\delta^{(2)} \leqslant \beta^{(2)}$ для всех $\delta^{(2)} \in \Delta^{(2)}$ и что $\left(G_{A^{(2)}}\right)_{\beta^{(2)}}(u) \subseteq\left(G_{A^{(1)}}\right)_{\beta^{(1)}}(u)$ для всех $u \in U^{(2)}$. Так как $\beta^{(1)} \leqslant \alpha^{(1)}$, то $\left(G_{A^{(1)}}\right)_{\beta^{(1)}}(u) \subseteq\left(G_{A^{(1)}}\right)_{\alpha^{(1)}}(u)$. Следовательно, $A$ - четкое или сильно четкое действие.

$\Leftarrow)$ Предположим, что вьполнены предположения, фигурирующие в условии (4.26.1). Мы должны показать, что $A$ - действие с нижней гранью или сильной нижней гранњю. Так как по предположению $A$ является четким действием, то найдется такой элемент $\delta^{(2)} \in \Phi_{A^{(2)}}$, что $\delta^{(2)} \leqslant \gamma^{(2)} \leqslant \beta^{(2)}$ для всех $\delta^{(2)} \in \Delta^{(2)}$ и $\left(G_{A^{(2)}}\right)_{\gamma^{(2)}}(u) \subseteq$ $\left(G_{A^{(1)}}\right)_{\beta^{(1)}}(u)$ для всех $u \in U^{(2)}$. Это означает, что $\gamma^{(2)} \in \Psi^{(2)}$. Однако, поскольку $\beta^{(2)}$ - инициальньй элемент в $\Psi^{(2)}$, то мы имеем $\beta^{(2)} \leqslant \gamma^{(2)}$ и, следовательно, $\left(G_{A^{(2)}}\right)_{\gamma^{(2)}}(u)=\left(G_{A^{(2)}}\right)_{\beta^{(2)}}(u)$. Поэтому $\left(G_{A^{(2)}}\right)_{\beta^{(2)}}(u) \subseteq\left(G_{A^{(1)}}\right)_{\beta^{(1)}}(u)$ для всех $u \in U^{(2)}$. Таким образом, $A$ - действие с нижней гранью или сильной нижней гранью.

Суммируем теперь несколько простых, но полезных фактов. 
ЛЕМMA 4.29 .

(4.29.1) Пусть $A$ - глобальное действие. Если $U \subseteq V \subseteq|A| u V$ - локальный репер, то множество $U$ также является локальным репером.

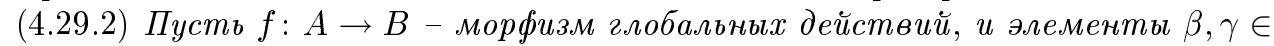
$\Phi_{(A, B)}$ таковь, ито $f(x) \in \beta(x) \cap \gamma(x)$ для всех $x \in|A|$. Предположим, что $\beta \leqslant \gamma$ и $(f, \gamma)$ является $A$-картой в $B$. Тогда $(f, \beta)$ - такжее $A$-карта в $B$.

(4.29.3) Пусть $A$ - относительное действие. Если множества $U^{(i)} \subseteq V^{(i)} \subseteq$ $\left|A^{(i)}\right|(i=1,2)$ таковь, что $U^{(2)} \subseteq U^{(1)}$ и пара $\left(V^{(1)}, V^{(2)}\right)$ является относительным локальным репером, то пара $\left(U^{(1)}, U^{(2)}\right)$ - также относительньи локальньи й репер.

(4.29.4) Пусть $f: A \rightarrow B$ - морфизм относительньх действий и әлементы $\beta^{(1)}, \gamma^{(1)} \in \Phi_{(A, B)^{(1)}}$ таковй, ито $f(x) \in \beta^{(1)}(x) \cap \gamma^{(1)}(x)$ для всех $x \in\left|A^{(1)}\right|$. Предположим, что $\beta \leqslant \gamma u(f, \gamma)$ является $A$-картой в $B$. Тогда $(f, \beta)$ - также A-карта в $B$.

Лемма 4.30. Пусть $B$ - некоторое относительное действие с нижней гранью (соответственно, сильной нижней гранью). Тогда для любого относительного действия $A$ морфизмы $\operatorname{Mor}(A, B)$ также образуют действие с нижней гранью (соответственно, сильной нижсней гранью).

ДоказАтельство. Так как $\operatorname{Mor}(A, B)^{(2)}=\operatorname{Mor}\left(A^{(1)}, B^{(2)}\right)$, то из леммы 3.22 вытекает, что $\operatorname{Mor}(A, B)^{(2)}$ является действием с нижней гранью (соответственно, с сильной нижней гранью).

Покажем теперь, что $\operatorname{Mor}(A, B)^{(1)}$ также обладает нижней гранью (соответственно, сильной нижней гранью). Пусть $U^{(1)} \subseteq\left|\operatorname{Mor}(A, B)^{(1)}\right|-$ некоторое конечное непустое подмножество. Обозначим через $\Delta^{(1)} \subseteq \Phi_{(A, B)^{(1)}}$ такое конечное подмножество, что для каждого $\delta \in \Delta^{(1)}$ пересечение $\left(X_{(A, B)^{(1)}}\right)_{\delta} \cap U^{(1)}$ не пусто. Положим $\Psi^{(1)}=\left\{\beta \in \Phi_{(A, B)^{(1)}} \mid U^{(1)}\right.$ есть $\beta$-репер, $\left.\delta \leqslant \beta \forall \delta \in \Delta^{(1)}\right\}$ и будем считать, что $\Psi^{(1)} \neq \varnothing$. Мы должны показать, что множество $\Psi^{(1)}$ имеет инициальньй элемент. Для каждого $x \in\left|A^{(1)}\right|$ положим $U^{(1)}(x)=\left\{f(x) \mid f \in U^{(1)}\right\}, \Delta^{(1)}(x)=\{\delta(x) \mid$ $\left.\delta \in \Delta^{(1)}\right\}$ и $\Psi^{(1)}(x)=\left\{\beta(x) \mid \beta \in \Psi^{(1)}\right\}$. По предположению для каждого $x \in\left|A^{(1)}\right|$ найдется такая координата $b_{x} \in \Phi_{B^{(1)}} \cup \Phi_{B^{(2)}}$, что $U^{(1)}(x)$ является $b_{x}$-репером и $\delta(x) \leqslant b_{x} \leqslant \beta(x)$ для всех $\delta \in \Delta^{(1)}$ и $\beta \in \Psi^{(1)}$. Аналогичньм образом, для каждого $x \in\left|A^{(2)}\right|$ существует такая координата $b_{x} \in \Phi_{B^{(2)}}$, что $U^{(1)}(x)$ является $b_{x}$-репером и $\delta(x) \leqslant b_{x} \leqslant \beta(x)$ для всех $\delta \in \Delta^{(1)}$ и $\beta \in \Psi^{(1)}$. Пусть $\gamma:\left|A^{(1)}\right| \rightarrow \Phi_{B^{(1)}} \cup \Phi_{B^{(2)}}$ - отображение, задаваемое правилом $x \mapsto b_{x}$. Ясно, что $\gamma \in \Phi_{(A, B)^{(1)}}$ и $\delta \leqslant \gamma \leqslant \beta$ для всех $\delta \in \Delta^{(1)}, \beta \in \Psi^{(1)}$. Рассмотрим какой-либо элемент $u \in U$. Чтобы завершить доказательство, достаточно показать, что пара $(u, \gamma)$ является $A$-картой. Пусть $\beta \in \Psi^{(1)}$. Так как $(u, \beta)$ является $A$-картой и $\gamma \leqslant \beta$, то из (4.29.4) следует, что $(u, \gamma)$ также является $A$-картой.

Докажем, наконец, что вьполнено условие (4.26.1). Пусть $\Delta^{(i)}, U^{(i)}$, и $\Psi^{(i)}$ такие же, как в (4.26.1). Для $x \in\left|A^{(1)}\right|$ определим множества $\Delta^{(i)}(x), U^{(i)}(x)$ и $\Psi^{(i)}(x)$ так же, как это было сделано несколькими строками вьше. При фиксированном $x$ набор $\Delta^{(i)}(x), U^{(i)}(x), \Psi^{(i)}(x)$ удовлетворяет условию (4.26.1) применительно к действию $B$. Пусть $b_{x}^{(i)} \in \Psi^{(i)}(x)$ - инициальный элемент, сушествование которого гарантируется этим условием. Положим

$$
\begin{aligned}
& \gamma^{(1)}:\left|A^{(1)}\right| \rightarrow \Phi_{B^{(1)}} \cup \Phi_{B^{(2)}}, \quad x \mapsto b_{x}^{(1)}, \\
& \gamma^{(2)}:\left|A^{(1)}\right| \rightarrow \Phi_{B}^{(2)}, \quad x \mapsto b_{x}^{(2)} .
\end{aligned}
$$


Ясно, что $\gamma^{(i)} \in \Phi_{(A, B)^{(i)}}, \delta^{(i)} \leqslant \gamma^{(i)} \leqslant \beta^{(i)}$ для всех $\delta^{(i)} \in \Delta^{(i)}, \beta^{(i)} \in \Psi^{(i)}$, и что $\left(G_{(A, B)^{(2)}}\right)_{\gamma^{(2)}}(u) \subseteq\left(G_{(A, B)^{(1)}}\right)_{\gamma^{(1)}}(u)$ для всех $u \in U^{(2)}$. Кроме того, для каждого $u \in U^{(1)}$ пара $\left(u, \gamma^{(1)}\right)$ является $A$-картой в $B$, поскольку для любого $\beta^{(1)}$ такова пара $\left(u, \beta^{(1)}\right)$; если же $u \in U^{(2)}$, то пара $\left(u, \gamma^{(2)}\right)$ является $A^{(1)}$-картой в $B^{(2)}$, поскольку для любого $\beta^{(2)} \in \Psi^{(2)}$ такова пара $\left(u, \beta^{(2)}\right)$.

Следуюшее утверждение является главным результатом этого параграфа.

ТЕОРема 4.31. Относительное действие с нижней гранью $\infty$-нормально и $\infty$-әкспоненциально. Относительное действие с сильной нижней гранью $\infty$-нормально и регулярно $\infty$-әкспоненциально.

ДокАЗАТЕЛЬСТво. Предположим, что действие $C$ обладает нижней гранью. Докажем, что $C$ является $\infty$-нормальньм. Согласно лемме 4.30 для этого достаточно показать, что $C$ является $A$-нормальным для любого относительного действия $A$. Пусть $g: B \rightarrow C$ - морфизм относительных действий. Так как $C^{(2)}$ как глобальное действие $\infty$-нормально, то морфизм $g: B^{(2)} \rightarrow C^{(2)}$ является $\infty$-нормальньм и поэтому $A^{(1)}$-нормальным. Пусть $(f, \beta)$ - некоторая $A$-карта в $B$ и $f=f_{0}, f_{1}, \ldots, f_{p}$ - произвольньй $A$-репер на $(f, \beta)$. Мы должны показать, что $g f_{0}, \ldots, g f_{p}$ является $A$-репером в $C$. Вначале мы построим такую относительную координату $\left(\gamma:\left|A^{(1)}\right| \rightarrow\right.$ $\left.\Phi_{C^{(1)}} \cup \Phi_{C^{(2)}}\right) \in \Phi_{(A, C)^{(1)}}$, для которой пара $(g f, \gamma)$ будет $A$-картой в $C$.

Положим $F^{(1)}=\left\{f_{0}, \ldots, f_{p}\right\}$. Для $x \in\left|A^{(1)}\right|$ обозначим через $F^{(1)}(x)$ множество $\left\{f_{0}(x), \ldots, f_{p}(x)\right\}$. Согласно локально-глобальной лемме 4.11 множество $F^{(1)}(x)$ является локальным репером в $B^{(1)}$ или $B^{(2)}$, в зависимости от того, находится ли $x$ в $\left|A^{(1) \backslash(2)}\right|$ или же в $\left|A^{(2)}\right|$. Следовательно, $g F^{(1)}(x)$ - локальный репер в $C^{(1)}$ или $C^{(2)}$ в зависимости от $x$. Пусть $\Psi^{(1)}(x)=\left\{c \in \Phi_{C^{(i)}} \mid g F^{(i)}(x)\right.$ является локальным репером в $C^{(i)}, i=1$, если же $x \in\left|A^{(1) \backslash(2)}\right|, i=2$, если же $\left.x \in\left|A^{(2)}\right|\right\}$. Поскольку действие $C^{(i)}$ обладает нижней гранью, множество $\Psi^{(1)}(x)$ имеет инициальньй элемент $c_{x}$. Определим отображение $\gamma:\left|A^{(1)}\right| \rightarrow \Phi_{C^{(1)}} \cup \Phi_{C^{(2)}}$ правилом $x \mapsto c_{x}$.

Покажем, что пара $(g f, \gamma)$ является $A$-картой в $C$. Пусть $\left(U^{(1)}, U^{(2)}\right)$ - полная пара локальных реперов в $A$. Тогда в силу леммы $3.24\left(F^{(1)} U^{(1)}, F^{(1)} U^{(2)}\right)$ является парой локальных реперов в $B$. Следовательно, $\left(g F^{(1)} U^{(1)}, g F^{(1)} U^{(2)}\right)-$ пара локальных реперов в $C$. Так как действие $C$ удовлетворяет условию (4.28), то пара $\left(g F^{(1)} U^{(1)}, g F^{(1)} U^{(2)}\right)$ представляет собой относительный локальный репер в некоторой точке $\left(c^{(1)}, c^{(2)}\right) \in \Phi_{C^{(1)}} \times \Phi_{C^{(2)}}$. Согласно (4.29.4), это означает, что $\left(g f U^{(1)}, g f U^{(2)}\right)$ - относительньй локальньй репер в $\left(c^{(1)}, c^{(2)}\right)$. Но из определения отображения $\gamma$, мы знаем, что для каждого $x \in U^{(1)} \backslash U^{(2)}$ (соответственно, $x \in U^{(2)}$ ) вьполнено соотношение $\gamma(x)=c_{x} \leqslant c^{(1)}$ (соответственно, $\left.\gamma(x)=c_{x} \leqslant c^{(2)}\right)$. Это показывает, что пара $(g f, \gamma)$ является $A$-картой в $C$.

Из определения $\gamma$ немедленно вытекает, что $g f=g f_{0}, g f_{1}, \ldots, g f_{p}$ есть $A$-репер на $(g f, \gamma)$. Тем самым, мы показали, что действие $C$ является $A$-нормальным.

Докажем теперь, что действие $C$ является $\infty$-экспоненщиальным. Пусть $A$ и $B$ - такие глобальные действия, что $\left|A^{(i)}\right|=\bigcup_{\alpha \in \Phi_{A^{(i)}}}\left(X_{A^{(i)}}\right)_{\alpha}$ и $\left|B^{(i)}\right|=\bigcup_{\beta \in \Phi_{B^{(i)}}}\left(X_{B^{(i)}}\right)_{\beta}$. Рассмотрим морфизм

$$
E: \operatorname{Mor}(A, \operatorname{Mor}(B, C)) \rightarrow \operatorname{Mor}(A \bowtie B, C),
$$

определенньй в 4.20 , и докажем, что он обладает $\infty$-нормальньм обратньм. По лемме 4.30 $\operatorname{Mor}(A, \operatorname{Mor}(B, C))$ является действием с нижней гранью и, стало быть, оно 
обязано быть $\infty$-нормальным. Таким образом, если обратньй морфизм для $E$ сушествует, то он $\infty$-нормален. Поэтому достаточно показать, что морфизм $E$ допускает обратньй. Существует очевидньй кандидат для этого обратного морфизма, а именно отображение множеств

$$
E^{\prime}:\left|\operatorname{Mor}(A \bowtie B, C)^{(1)}\right| \rightarrow(A,(B, C)), \quad f \mapsto E^{\prime} f,
$$

где $\left(E^{\prime} f(x)\right)(y)=f(x, y)$. Покажем, что $E^{\prime} f \in\left|\operatorname{Mor}(A, \operatorname{Mor}(B, C))^{(1)}\right|$ и что результируюшее отображение $E^{\prime}:\left|\operatorname{Mor}(A \bowtie B, C)^{(1)}\right| \rightarrow\left|\operatorname{Mor}(A, \operatorname{Mor}(B, C))^{(1)}\right|$ определяет морфизм глобальных действий $\operatorname{Mor}(A \bowtie B, C)^{(1)} \rightarrow \operatorname{Mor}(A, \operatorname{Mor}(B, C))^{(1)}$. Из теоретико-множественных свойств $E^{\prime}$ будет следовать, что $E^{\prime}$ является обратным для $E$.

Вначале докажем, что отображение $E^{\prime} f:\left|A^{(1)}\right| \rightarrow(B, C)$ определяет морфизм глобальных действий $A^{(1)} \rightarrow \operatorname{Mor}(B, C)^{(1)}$. Нам нужно проверить два свойства:

1) если $x \in\left|A^{(1)}\right|$, то $E^{\prime} f(x):\left|B^{(1)}\right| \rightarrow|C|^{(1)}, y \mapsto\left(E^{\prime} f(x)\right)(y)$ определяет морфизм относительных действий $B \rightarrow C$;

2) результируюшее отображение $E^{\prime} f:\left|A^{(1)}\right| \rightarrow\left|\operatorname{Mor}(B, C)^{(1)}\right|, x \mapsto E^{\prime} f(x)$ определяет морфизм глобальных действий $A^{(1)} \rightarrow \operatorname{Mor}(B, C)^{(1)}$.

Доказательство того, что правило $y \mapsto\left(E^{\prime} f(x)\right)(y)$ задает морфизм глобальных действий $B^{(1)} \rightarrow C^{(1)}$, в точности совпадает с аналогичным доказательством из теоремы 3.23. Более того, очевидно, что морфизм $E^{\prime} f(x): B^{(1)} \rightarrow C^{(1)}$ переводит $\left|B^{(2)}\right|$ в $\left|C^{(2)}\right|$. Поэтому, повторяя приведенные вьше рассуждения, мы получаем, что $\left.E^{\prime} f(x)\right|_{\left|B^{(2)}\right|}$ является морфиимом глобальных действий $B^{(2)} \rightarrow C^{(2)}$. Следовательно, $E^{\prime} f(x): B \rightarrow C$ - морфизм относительных действий.

Пусть теперь $x_{0}, \ldots, x_{p}$ - локальный репер в $A^{(1)}$. Мы должны показать, что $E^{\prime} f\left(x_{0}\right), \ldots, E^{\prime} f\left(x_{p}\right)$ - локальньй репер в $\operatorname{Mor}(B, C)^{(1)}$. Каждьй элемент в $y \in\left|B^{(1)}\right|$ служит локальным репером в $B^{(1)}$, поскольку $\left|B^{(1)}\right|=\bigcup_{\beta \in \Phi_{B^{(1)}}}\left(X_{B^{(1)}}\right)_{\beta}$. Следовательно, $\left(x_{0}, y\right), \ldots,\left(x_{p}, y\right)$ - локальньй репер в $(A \bowtie B)^{(1)}$. Но это означает, что множество $f\left(x_{0}, y\right), \ldots, f\left(x_{p}, y\right)$ является локальным репером в $C^{(1)}$, причем если $y \in B^{(2)}$, то $f\left(x_{0}, y\right), \ldots, f\left(x_{p}, y\right)$ - локальньй репер в $C^{(2)}$. Из существования для действия $C^{(1)}$ нижней грани вытекает, что при $y \in B^{(1) \backslash(2)}$ множество $\left\{c \in \Phi_{C^{(1)}} \mid f\left(x_{0}, y\right), \ldots, f\left(x_{p}, y\right)\right.$ есть $c$-репер $\}$ имеет инициальньй элемент $c_{y}$. Аналогично, из существования нижней грани для $C^{(2)}$ следует, что при $y \in\left|B^{(2)}\right|$ множество $\left\{c \in \Phi_{C^{(2)}} \mid f\left(x_{0}, y\right), \ldots, f\left(x_{p}, y\right)\right.$ есть $c$-репер $\}$ также имеет инициальньй элемент; мы вновь обозначим его через $c_{y}$. Определим отображение $\gamma:\left|B^{(1)}\right| \rightarrow \Phi_{C^{(1)}} \cup \Phi_{C^{(2)}}$ правилом $y \mapsto c_{y}$. Нам нужно показать, что пара $\left(E^{\prime} f\left(x_{0}\right), \gamma\right)$ является $B$-картой в $C$. Предположим на мгновение, что это уже доказано. Тогда из локально-глобальной леммы 4.11 будет следовать, что $E^{\prime} f\left(x_{0}\right), \ldots, E^{\prime} f\left(x_{p}\right)$ является $B$-репером на $\left(E^{\prime} f\left(x_{0}\right), \gamma\right)$. Но тогда, по определению, $E^{\prime} f\left(x_{0}\right), \ldots, E^{\prime} f\left(x_{p}\right)$ - локальный репер в $\operatorname{Mor}(B, C)^{(1)}$, что мы и хотели показать.

Докажем, что $\left(E^{\prime} f\left(x_{0}\right), \gamma\right)$ является $B$-картой в $C$. Пусть $\left(V^{(1)}, V^{(2)}\right)$ - полная пара локальных реперов в $B$. Мы должны показать, что $\left(E^{\prime} f\left(x_{0}\right) V^{(1)}, E^{\prime} f\left(x_{0}\right) V^{(2)}\right)-$ такой относительньй локальньй репер в некоторой точке $\left(c^{(1)}, c^{(2)}\right) \in\left(\Phi_{C^{(1)}}, \Phi_{C^{(2)}}\right)$, что $\gamma(y) \leqslant c^{(1)}$ для всех $y \in V^{(1)} \backslash V^{(2)}$ и $\gamma(y) \leqslant c^{(2)}$ для всех $y \in V^{(2)}$. Пусть $U=\left\{x_{0}, \ldots, x_{p}\right\}$. Тогда $\left(U \times V^{(1)}, U \times V^{(2)}\right)$ является парой локальных реперов в $A \bowtie B$. Стало быть $\left(f\left(U \times V^{(1)}\right), f\left(U \times V^{(2)}\right)\right)$ - пара локальных реперов в $C$. Так как $\left(f\left(U \times V^{(1)}\right), f\left(U \times V^{(2)}\right)\right)=\left(\left(E^{\prime} f\right) U V^{(1)},\left(E^{\prime} f\right) U V^{(2)}\right)$, то эта последняя 
пара является парой локальных реперов в $C$. Из условия (4.26.1) следует, что $\left(\left(E^{\prime} f\right) U V^{(1)},\left(E^{\prime} f\right) U V^{(2)}\right)$ - относительньй локальньй репер в некоторой точке $\left(c^{(1)}, c^{(2)}\right) \in \Phi_{C^{(1)}} \times \Phi_{C^{(2)}}$. Поэтому $\left(\left(E^{\prime} f\right)\left(x_{0}\right) V^{(1)},\left(E^{\prime} f\right)\left(x_{0}\right) V^{(2)}\right)$ также является относительным локальным репером в этой точке. Но из определения отображения $\gamma$, вытекает, что для $y \in V^{(1)} \backslash V^{(2)} \gamma(y)=c_{y} \leqslant c^{(1)}$ и для $y \in V^{(2)} \gamma(y)=c_{y} \leqslant c^{(2)}$.

Ясно, что морфизм

$$
E^{\prime} f: A^{(1)} \rightarrow \operatorname{Mor}(B, C)^{(1)}
$$

переводит $\left|A^{(2)}\right|$ в $\left|\operatorname{Mor}(B, C)^{(2)}\right|$. Покажем, что функция

$$
\left.\left(E^{\prime} f\right)\right|_{\left|A^{(2)}\right|}:\left|A^{(2)}\right| \rightarrow\left|\operatorname{Mor}(B, C)^{(2)}\right|
$$

определяет морфизм глобальных действий $A^{(2)} \rightarrow \operatorname{Mor}(B, C)^{(2)}$. Этим фактом будет завершено доказательство того, что $E^{\prime} f: A \rightarrow \operatorname{Mor}(B, C)$ является морфизмом относительных действий. Заметим, прежде всего, что $\operatorname{Mor}(B, C)^{(2)}=\operatorname{Mor}\left(B^{(1)}, C^{(2)}\right)$. Прямая проверка показывает, что функцию $\left.\left(E^{\prime} f\right)\right|_{\left|A^{(2)}\right|}$ можно отождествить с функцией $E^{\prime}\left(\left.f\right|_{A^{(2)} \times B^{(1)}}\right)$, где

$$
E^{\prime}: \operatorname{Mor}\left(A^{(2)} \times B^{(2)}, C^{(2)}\right) \rightarrow \operatorname{Mor}\left(A^{(2)}, \operatorname{Mor}\left(B^{(1)} \times C^{(2)}\right)\right)
$$

- морфизм, построенньй при доказательстве теоремы 3.23. Но в силу этой теоремы $E^{\prime}\left(\left.f\right|_{A^{(2)} \times B^{(2)}}\right)-$ морфизм глобальных действий.

Докажем теперь, что функция

$$
E^{\prime}:\left|\operatorname{Mor}(A \bowtie B, C)^{(1)}\right| \rightarrow\left|\operatorname{Mor}(A, \operatorname{Mor}(B, C))^{(1)}\right|
$$

определяет морфизм относительных действий

$$
\operatorname{Mor}(A \bowtie B, C) \rightarrow \operatorname{Mor}(A, \operatorname{Mor}(B, C)) .
$$

Установим вначале, что $E^{\prime}$ задает морфизм глобальных действий

$$
\operatorname{Mor}(A \bowtie B, C)^{(1)} \rightarrow \operatorname{Mor}(A, \operatorname{Mor}(B, C))^{(1)} .
$$

Пусть $f=f_{0}, f_{1}, \ldots, f_{p}$-локальньй репер в $\operatorname{Mor}(A \bowtie B, C)^{(1)}$. Мы должныпоказать, что множество $E^{\prime} f_{0}, \ldots, E^{\prime} f_{p}$ является локальным репером в $\operatorname{Mor}(A, \operatorname{Mor}(B, C))^{(1)}$. Согласно локально-глобальной лемме 4.11 для каждого элемента $(x, y)$ из $A \times B$ множество $f_{0}(x, y), \ldots, f_{p}(x, y)$ являются локальньм репером в $C^{(1)}$. Кроме того, если $(x, y) \in X_{(A \bowtie B)^{(2)}}$, то $f_{0}(x, y), \ldots, f_{p}(x, y)$ - локальньй репер и в $C^{(2)}$. Из сушествования у действия $C^{(1)}$ нижней грани следует, что для $(x, y) \in(A \bowtie B)^{(1) \backslash(2)}$, множество $\left\{c \in \Phi_{C^{(1)}} \mid f_{0}(x, y), \ldots, f_{p}(x, y)\right.$ есть $c$-репер $\}$ имеет инициальньй элемент $c_{(x, y)}$. Аналогично, если $(x, y) \in X_{(A \bowtie B)^{(2)}}$, то множество $\left\{c \in \Phi_{C^{(2)}} \mid\right.$ $f_{0}(x, y), \ldots, f_{p}(x, y)$ есть $c$-репер $\}$ обладает инициальньм элементом, который мы также обозначим через $c_{(x, y)}$. Определим отображение $\gamma:|A| \rightarrow\left(|B|, \Phi_{C^{(1)}} \cup \Phi_{C^{(2)}}\right)$ правилом $x \mapsto c_{\left(x,{ }_{-}\right)}$. Мы утверждаем, что пара $\left(E^{\prime} f_{0}, \gamma\right)$ является $A$-картой в $\operatorname{Mor}(B, C)$. Тогда из определения $\gamma$ и леммы 4.11 будет следовать, что множество $E^{\prime} f_{0}, \ldots, E^{\prime} f_{p}$ является $A$-репером на $\left(E^{\prime} f, \gamma\right)$. Но это означает, что $E^{\prime} f_{0}, \ldots, E^{\prime} f_{p}-$ локальньй репер в $\operatorname{Mor}(A, \operatorname{Mor}(B, C))^{(1)}$, что и требуется.

Пусть $\left(U^{(1)}, U^{(2)}\right)$ - полная пара локальных реперов в $A$. Мы должны показать, что $\left(\left(E^{\prime} f\right) U^{(1)},\left(E^{\prime} f\right) U^{(2)}\right)$ - относительный локальный репер в некоторой точке 
$\left(\delta^{(1)} \times \delta^{(2)}\right) \in \Phi_{(B, C)^{(1)}} \times \Phi_{(B, C)^{(2)}}$, такой, что $\gamma(x) \leqslant \delta^{(1)}$ для всех $x \in U^{(1)} \backslash U^{(2)}$ и $\gamma(x) \leqslant \delta^{(2)}$ для всех $x \in U^{(2)}$. Пусть $F=\left\{f=f_{0}, \ldots, f_{p}\right\}$. Так как $F-$ локальньй репер в $\operatorname{Mor}(A \bowtie B, C)^{(1)}$, то найдется такая $A \bowtie B$-карта $\left(f_{0}, \varepsilon\right)$ в $C$, что $F$ есть $A \bowtie B$-репер на $\left(f_{0}, \varepsilon\right)$. Для любого фиксированного $y \in\left|B^{(1) \backslash(2)}\right|$ пара $\left(f_{0}(, y), \varepsilon(, y)\right)$ является $A$-картой в $C$, а множество $F(, y)=\left\{f_{0}(, y), \ldots, f_{p}(, y)\right\}$ есть $A$-репер на этой карте. Если же $y \in\left|B^{(2)}\right|$, то $\left(f_{0}(, y), \varepsilon(, y)\right)$ является $A^{(1)}$-картой в $C^{(2)}$ и множество $f_{0}(, y), \ldots, f_{p}(, y)$ есть $A^{(1)}$-репер на ней. Поскольку $\left(U^{(1)}, U^{(2)}\right)$ - пара локальных реперов в $A$, из леммы 3.24 вытекает, что $\left(F\left(U^{(1)}, y\right), F\left(U^{(2)}, y\right)\right)$ - пара локальных реперов в $C$. А так как действие $C$ обладает нижней гранью, то пара $\left(F\left(U^{(1)}, y, F\left(U^{(2)}, y\right)\right.\right.$ представляет собой относительный локальный репер в некоторой точке $\left(d_{y}^{(1)}, d_{y}^{(2)}\right) \in\left(\Phi_{C^{(1)}} \cup \Phi_{C^{(2)}}\right) \times \Phi_{C^{(2)}}$, такой что $d_{y}^{(1)} \in \Phi_{C^{(1)}}$, если $y \in\left|B^{(1) \backslash(2)}\right|$ и $d_{y}^{(1)} \in \Phi_{C^{(2)}}$, если $y \in\left|B^{(2)}\right|$. Таким образом, в силу леммы (4.29.4) пара $\left(f\left(U^{(1)}, y\right), f\left(U^{(2)}, y\right)\right)$ является относительным локальньм репером в точке $\left(d_{y}^{(1)}, d_{y}^{(2)}\right)$. Положим

$$
\begin{aligned}
& \delta^{(1)}: B^{(1)} \rightarrow \Phi_{C^{(1)}} \cup \Phi_{C^{(2)}}, \quad y \mapsto d_{y}^{(1)}, \\
& \delta^{(2)}: B^{(2)} \rightarrow \Phi_{C^{(2)}}, y \mapsto d_{y}^{(2)} .
\end{aligned}
$$

Согласно локально-глобальным леммам 3.7 и 4.11 пара $\left(\left(E^{\prime} f\right) U^{(1)},\left(E^{\prime} f\right) U^{(2)}\right)$ будет относительным локальным репером в точке $\left(\delta^{(1)}, \delta^{(2)}\right)$ тогда и только тогда, когда $\left(\left(E^{\prime} f\right)(u), \delta^{(1)}\right)$ будет $B$-картой в $C$ для любого $u \in U^{(1)}$, а $\left(\left(E^{\prime} f\right)(u), \delta^{(2)}\right)$ будет $B^{(1)}$-картой в $C^{(2)}$ для любого $u \in U^{(2)}$. Поэтому все, что нам осталось, это доказать, что $\left(\left(E^{\prime} f\right)(u), \delta^{(1)}\right)$ и $\left(\left(E^{\prime} f\right)(u), \delta^{(2)}\right)$ - соответствуюшие карты. В самом деле, коль скоро это доказано, то из определения отображения $\gamma$ следует, что $\gamma(x) \leqslant \delta^{(1)}$ для всех $x \in U^{(1)} \backslash U^{(2)}$ и $\gamma(x) \leqslant \delta^{(2)}$ для всех $x \in U^{(2)}$.

Итак, покажем, что пара $\left(\left(E^{\prime} f\right)(u), \delta^{(1)}\right)$ является $B$-картой в $C$ для любого $u \in U^{(1)}$. Пусть $\left(V^{(1)}, V^{(2)}\right)$ - полная пара локальных реперов в $B$. Мы должны доказать, что $\left(\left(E^{\prime} f\right)(u) V^{(1)},\left(E^{\prime} f\right)(u) V^{(2)}\right)$ является относительным локальным репером в некоторой точке $\left(c^{(1)}, c^{(2)}\right) \in \Phi_{C^{(1)}} \times \Phi_{C^{(2)}}$, такой что $\delta^{(1)}(y) \leqslant c^{(1)}$ для всех $y \in V^{(1)} \backslash V^{(2)}$ и $\delta^{(1)}(y) \leqslant c^{(2)}$ для всех $y \in V^{(2)}$. Из леммы 3.24 следует, что $\left(F\left(U, V^{(1)}\right), F\left(U, V^{(2)}\right)\right)$ является парой локальных реперов в $C$. Значит, и $\left(\left(E^{\prime} F\right) U V^{(1)}\right.$, и $\left.\left(E^{\prime} F\right) U V^{(2)}\right)$ - пара локальных реперов в $C$. Так как действие $C$ обладает нижней гранью, то $\left(\left(E^{\prime} F\right) U V^{(1)},\left(E^{\prime} F\right) U V^{(2)}\right)$ - относительньй локальньй репер в некоторой точке $\left(c^{(1)}, c^{(2)}\right) \in \Phi_{C^{(1)}} \times \Phi_{C^{(2)}}$. А поскольку вьполнено условие (4.26.1), то $\delta^{(1)}(y) \leqslant c^{(1)}$ для всех $y \in V^{(1)} \backslash V^{(2)}$ и $\delta^{(2)}(y) \leqslant c^{(2)}$ для всех $y \in$ $V^{(2)}$. Но по лемме $(4.29 .4)$ пара $\left(\left(E^{\prime} f\right)(u) V^{(1)},\left(E^{\prime} f\right)(u) V^{(2)}\right)$ является относительньм локальным репером в точке $\left(c^{(1)}, c^{(2)}\right)$. Следовательно, $\left(\left(E^{\prime} f\right)(u), \delta^{(1)}\right)$ является $B$-картой в $C$ для любого $u \in U^{(1)}$.

Тот факт, что $\left(\left(E^{\prime} f\right)(u), \delta^{(2)}\right)$ является $B^{(1)}$-картой в $C^{(2)}$ для любого $u \in U^{(2)}$ доказывается так же, как в теореме 3.23 . Этим завершается доказательство того, что $E^{\prime}: \operatorname{Mor}(A \bowtie B, C)^{(1)} \rightarrow \operatorname{Mor}(A, \operatorname{Mor}(B, C))^{(1)}$ есть морфизм глобальных действий.

Легко видеть, что этот морфизм переводит $\left|\operatorname{Mor}(A \bowtie B, C)^{(2)}\right|$ в $\left(A^{(2)},\left(B^{(1)}, C^{(2)}\right)\right)$. В самом деле,

$$
\operatorname{Mor}(A \bowtie B, C)^{(2)}=\operatorname{Mor}\left(A^{(1)} \times B^{(1)}, C^{(2)}\right),
$$

а отображение $\left.E^{\prime}\right|_{\left|\operatorname{Mor}\left(A^{(1)} \times B^{(1)}, C^{(1)}\right)\right|}$ совпадает с отображением $\mid \operatorname{Mor}\left(A^{(1)} \times\right.$ $\left.B^{(1)}, C^{(2)}\right)|\rightarrow| \operatorname{Mor}\left(A^{(2)}, \operatorname{Mor}\left(B^{(1)}, C^{(2)}\right)\right) \mid$, индуцированным морфизмом глобаль- 
ных действий $E^{\prime}: \operatorname{Mor}\left(A^{(1)} \times B^{(1)}, C^{(2)}\right) \rightarrow \operatorname{Mor}\left(A^{(2)}, \operatorname{Mor}\left(B^{(1)}, C^{(2)}\right)\right)$ из теоремы 3.23. Следовательно, $E^{\prime}: \operatorname{Mor}(A \bowtie B, C)^{(2)} \rightarrow \operatorname{Mor}(A, \operatorname{Mor}(B, C))^{(2)}$ является морфизмом глобальных действий, а это, в свою очередь, означает, что $E^{\prime}: \operatorname{Mor}(A \bowtie B, C) \rightarrow \operatorname{Mor}(A, \operatorname{Mor}(B, C))$ - морфизм относительных действий. Тем самым, мы доказали, что действие $C$ обладает свойством $\infty$-экспоненщиальности.

Предположим, наконец, что $C$ - действие с сильной нижней гранью. Мы должны показать, что $C$ регулярно $\infty$-экспоненциально. Для этого нужно убедиться, что морфизм

$$
E: \operatorname{Mor}(A, \operatorname{Mor}(B, C)) \rightarrow \operatorname{Mor}(A \bowtie B, C)
$$

имеет регулярньй обратный $E^{\prime}$. Существуют очевидные кандидаты для структурных компонент $\left(E_{\Phi}^{\prime}, E_{G}^{\prime}, E_{X}^{\prime}\right)$ морфизма $E^{\prime}$. Прежде всего положим,

$$
E_{X}^{\prime}: \operatorname{Mor}(A \bowtie B, C)|\longrightarrow| \operatorname{Mor}(A, \operatorname{Mor}(B, C)) \mid, \quad f \longmapsto E^{\prime} f,
$$

где $f \mapsto E^{\prime} f$ - построенное выше отображение. Определим отображение

$$
E_{\Phi^{(i)}}^{\prime}: \Phi_{(A \bowtie B, C)^{(i)}} \longrightarrow \Phi_{(A,(B, C))^{(i)}} \quad(i=1,2)
$$

как обратное для отображения множеств $E_{\Phi^{(i)}}$ (см. (3.16)). И, наконец, зададим естественные преобразования

$$
E_{G^{(i)}}^{\prime}: G_{(A \bowtie B, C)^{(i)}} \longrightarrow\left(G_{(A,(B, C))^{(i)}}\right)_{E_{\Phi^{(i)}}^{\prime}()} \quad(i=1,2)
$$

следуюшим образом. Для $\alpha \in \Phi_{(A \bowtie B, C)^{(1)}}$ определим групповой гомоморфизм

$$
E_{G^{(1)}}^{\prime}(\alpha):\left(G_{(A \bowtie B, C)^{(1)}}\right)_{\alpha} \longrightarrow\left(G_{(A,(B, C))^{(1)}}\right)_{E_{\Phi^{(1)}}^{\prime}(\alpha)}
$$

так, чтобы он тождественно отображал сомножитель из

$$
\left(G_{(A \bowtie B, C)^{(1)}}\right)_{\alpha}=\prod_{(x, y) \in\left|A^{(1) \backslash(2)}\right| \times\left|B^{(1) \backslash(2)}\right|}\left(G_{C^{(1)}}\right)_{\alpha(x, y)} \times \prod_{(x, y) \in\left|(A \bowtie B)^{(2)}\right|}\left(G_{C^{(2)}}\right)_{\alpha(x, y)}
$$

с индексом $\alpha(x, y)$ на сомножитель из

$$
\begin{aligned}
\left(G_{(A,(B, C))^{(1)}}\right)_{E_{\Phi^{(1)}}^{\prime}(\alpha)}= & \prod_{x \in \mid A^{(1) \backslash(2) \mid}}\left[\prod_{y \in\left|B^{(1) \backslash(2)}\right|}\left(G_{C^{(1)}}\right)_{\left(E_{\Phi^{(1)}}^{\prime} \alpha\right)(x)(y)}\right. \\
& \left.\left.\times \prod_{y \in\left|B^{(2)}\right|}\left(G_{C^{(2)}}\right)_{\left(E_{\Phi^{(1)}}^{\prime}\right.} \alpha\right)(x)(y)\right] \\
& \times \prod_{x \in\left|A^{(2)}\right|} \prod_{y \in\left|B^{(1)}\right|}\left(G_{C^{(2)}}\right)_{\left(E_{\Phi^{(1)}}^{\prime} \alpha\right)(x)(y)}
\end{aligned}
$$

с индексом $\left(E_{\Phi^{(1)}}^{\prime} \alpha\right)(x)(y)$. Для $\alpha \in \Phi_{(A \bowtie B, C)^{(2)}}$, определим гомоморфизм групп

$$
E_{G^{(2)}}^{\prime}(\alpha):\left(G_{(A \bowtie B, C)^{(2)}}\right)_{\alpha} \rightarrow\left(G_{(A,(B, C))^{(2)}}\right)_{E_{\Phi^{(2)}}^{\prime}(\alpha)}
$$

так, чтобы он тождественно отображал сомножитель из

$$
\left(G_{(A \bowtie B, C)^{(2)}}\right)_{\alpha}=\prod_{(x, y) \in\left|A^{(1)}\right| \times\left|B^{(1)}\right|}\left(G_{C^{(2)}}\right)_{\alpha(x, y)}
$$


с индексом $\alpha(x, y)$ на сомножитель из

$$
\left.\left(G_{\left.(A,(B, C))^{(2)}\right)}\right)_{E_{\Phi^{(2)}}^{\prime}(\alpha)}=\prod_{x \in\left|A^{(1)}\right|}\left(\prod_{y \in\left|B^{(1)}\right|}\left(G_{C^{(2)}}\right)_{\left(E_{\Phi^{(2)}}^{\prime}\right.} \alpha\right)(x)(y)\right)
$$

с индексом $\left(E_{\Phi^{(2)}}^{\prime} \alpha\right)(x)(y)$.

Из всех этих свойств регулярного морфизма для $E^{\prime}$ не очевидно лишь включение $E_{X}^{\prime}\left(X_{(A \bowtie B, C)^{(i)}}\right)_{\alpha} \subseteq\left(X_{(A,(B, C))^{(i)}}\right)_{E_{\Phi^{(i)}}^{\prime}(\alpha)}$, где $\alpha \in \Phi_{(A \bowtie B, C)^{(i)}}(i=1,2)$.

Мы докажем это включение для $i=1$. Нам нужно показать, что если $(f, \alpha)-$ некоторая $(A \bowtie B)$-карта в $C$, то $\left(E_{X}^{\prime}(f), E_{\Phi^{(1)}}^{\prime}(\alpha)\right)$ является $A$-картой в $\operatorname{Mor}(B, C)$.

Пусть $\left(U^{(1)}, U^{(2)}\right)$ - полная пара локальных реперов. Мы должны убедится, что $\left(\left(E^{\prime} f\right) U^{(1)},\left(E^{\prime} f\right) U^{(2)}\right)$ является относительным локальным репером в некоторой точке $\left(\gamma^{(1)}, \gamma^{(2)}\right) \in \Phi_{(B, C)^{(1)}} \times \Phi_{(B, C)^{(2)}}$ такой, что $\gamma^{(1)} \geqslant\left(E_{\Phi^{(1)}}^{\prime} \alpha\right)(x)$ для всех $x \in U^{(2)}$. С этой целью построим отображения $\gamma^{(1)}$ и $\gamma^{(2)}$. Положим, прежде всего,

$$
\begin{aligned}
& \Psi^{(1)}(y)=\left\{c \in \Phi_{C^{(1)}} \mid f\left(U^{(1)} \times\{y\}\right) \text { есть } c \text {-репер, } c \geqslant \alpha(x, y)\right. \\
& \left.\forall x \in U^{(1)} \backslash U^{(2)}\right\}, \quad y \in B^{(1) \backslash(2)} ; \\
& \Psi^{(1)}(y)=\left\{c \in \Phi_{C^{(2)}} \mid f\left(U^{(1)} \times\{y\}\right) \text { есть } c \text {-репер, } c \geqslant \alpha(x, y)\right. \\
& \left.\forall x \in U^{(1)} \backslash U^{(2)}\right\}, \quad y \in B^{(2)} ; \\
& \Psi^{(2)}(y)=\left\{c \in \Phi_{C^{(2)}} \mid f\left(U^{(2)} \times\{y\}\right) \text { есть } c \text {-репер, } c \geqslant \alpha(x, y)\right. \\
& \left.\forall x \in U^{(2)}\right\}, \quad y \in B^{(1)} .
\end{aligned}
$$

Из существования у действий $C^{(i)}(i=1,2)$ сильной нижней грани следует, что множества $\Psi^{(1)}(y)$ и $\Psi^{(2)}(y)$ имеют инициальные элементы $c_{y}^{(1)}$ и $c_{y}^{(2)}$, соответственно. Определим отображения $\gamma^{(1)}$ и $\gamma^{(2)}$, полагая

$$
\begin{aligned}
& \gamma^{(1)}:\left|B^{(1)}\right| \rightarrow \Phi_{C^{(1)}} \cup \Phi_{C^{(2)}}, \quad y \mapsto c_{y}^{(1)} ; \\
& \gamma^{(2)}:\left|B^{(1)}\right| \rightarrow \Phi_{C^{(2)}}, \quad y \mapsto c_{y}^{(2)} .
\end{aligned}
$$

По построению, $\gamma^{(1)} \geqslant\left(E_{\Phi^{(1)}}^{\prime} \alpha\right)(x)$ для всех $x \in U^{(1)} \backslash U^{(2)}$ и $\gamma^{(2)} \geqslant\left(E_{\Phi^{(1)}}^{\prime} \alpha\right)(x)$ для всех $x \in U^{(2)}$. Предположим на мгновение, что мы доказали следующий факт: для любого $x \in U^{(2)}$ пара $\left(\left(E^{\prime} f\right)(x), \gamma^{(1)}\right)$ является $B$-картой в $C$ и пара $\left(\left(E^{\prime} f\right)(x), \gamma^{(2)}\right)$ является $B^{(1)}$-картой в $C^{(2)}$. Тогда из определения $\gamma^{(1)}$, следует, что $\left(E^{\prime} f\right) U^{(1)} \subseteq\left(G_{(B, C)^{(1)}}\right)_{\gamma^{(1)}}\left(E^{\prime} f\right)(x)$ и и $\left(E^{\prime} f\right) U^{(2)} \subseteq\left(G_{(B, C)^{(2)}}\right)_{\gamma^{(2)}}\left(E^{\prime} f\right)(x)$, т.е. что $\left(E^{\prime} f\right) U^{(1)}$ - локальньй репер в $\gamma^{(1)}$, а $\left(E^{\prime} f\right) U^{(2)}$ - локальньй репер в $\gamma^{(2)}$. Кроме того, из сушествования у действия $C$ сильной нижней грани вытекает, что $\left(G_{C^{(2)}}\right)_{\gamma^{(2)}(y)}\left(E^{\prime} f\right)(x, y) \subseteq\left(G_{C^{(1)}}\right)_{\gamma^{(1)}(y)}\left(E^{\prime} f\right)(x, y)$ для любых $x \in U^{(2)}$ и $y \in\left|B^{(1)}\right|$. Стало быть, $\left(G_{(B, C)^{(2)}}\right)_{\gamma^{(2)}}\left(E^{\prime} f\right)(x) \subseteq\left(G_{(B, C)^{(1)}}\right)_{\gamma^{(1)}}\left(E^{\prime} f\right)(x)$. Это означает, что пара $\left(\left(E^{\prime} f\right) U^{(1)},\left(E^{\prime} f\right) U^{(2)}\right)$ - относительньй локальньй репер в точке $\left(\gamma^{(1)}, \gamma^{(2)}\right)$, причем $\gamma^{(1)} \geqslant\left(E_{\Phi^{(1)}}^{\prime} \alpha\right)(x)$ для всех $x \in U^{(1)} \backslash U^{(2)}$ и $\gamma^{(2)} \geqslant\left(E_{\Phi^{(1)}}^{\prime} \alpha\right)(x)$ для всех $x \in U^{(2)}$. 
Покажем теперь, что для любого $x \in U^{(1)}$ пара $\left(\left(E^{\prime} f\right)(x), \gamma^{(1)}\right)$ является $B$-картой в $C$. Пусть $\left(V^{(1)}, V^{(2)}\right)$ - полная пара локальных реперов в $B$. Мы должны доказать, что $\left(\left(E^{\prime} f\right)(x) V^{(1)},\left(E^{\prime} f\right)(x) V^{(2)}\right)$ - относительньй локальный репер в некоторой точке $\left(c^{(1)}, c^{(2)}\right) \in \Phi_{C^{(1)}} \times \Phi_{C^{(2)}}$ такой, что $c^{(1)} \geqslant \gamma^{(1)}(y)$ для всех $y \in V^{(1)} \backslash V^{(2)}$ и $c^{(2)} \geqslant$ $\gamma^{(2)}(y)$ для всех $y \in V^{(2)}$. Так как $\left(U^{(1)} \times V^{(1)}, U^{(1)} \times V^{(2)}\right)$ является парой локальных реперов в $A \bowtie B$, то $\left(\left(E^{\prime} f\right) U^{(1)} V^{(1)},\left(E^{\prime} f\right) U^{(1)} V^{(2)}\right)=\left(f\left(U^{(1)} \times V^{(1)}\right), f\left(U^{(1)} \times V^{(2)}\right)\right)$ - пара локальных реперов в $C$. Положим $\Delta^{(1)}=\left\{\alpha(x, y) \mid x \in U^{(1)} \backslash U^{(2)}, y \in\right.$ $\left.B^{(1) \backslash(2)}\right\}$ и $\Delta^{(2)}=\left\{\alpha(x, y) \mid x \in U^{(1)} \backslash U^{(2)}, y \in B^{(2)}\right\}$. (Если $U^{(1)} \backslash U^{(2)}=\varnothing$, то разумеется $\Delta^{(1)}=\varnothing=\Delta^{(2)}$.) Из существования у действия $C$ сильной нижней грани следует, что $\left(\left(E^{\prime} f\right) U^{(1)} V^{(1)},\left(E^{\prime} f\right) U^{(1)} V^{(2)}\right)$ - относительньй локальньй репер в некоторой точке $\left(c^{(1)}, c^{(2)}\right) \in \Phi_{C^{(1)}} \times \Phi_{C^{(2)}}$ такой, что $c^{(1)} \geqslant \alpha(x, y)$ для всех $\alpha(x, y) \in$ $\Delta^{(1)}$ и $c^{(2)} \geqslant \alpha(x, y)$ для всех $\alpha(x, y) \in \Delta^{(2)}$. По построению $\gamma^{(1)}$, мы имеем $c^{(1)} \geqslant$ $\gamma^{(1)}(y)$ для всех $y \in V^{(1)} \backslash V^{(2)}$ и $c^{(2)} \geqslant \gamma^{(1)}(y)$ для всех $y \in V^{(2)}$.

Покажем, что для $x \in U^{(2)}$ пара $\left(\left(E^{\prime} f\right)(x), \gamma^{(2)}\right)$ является $B^{(1)}$-картой в $C^{(2)}$. Пусть $V$ - локальньй репер в $B^{(1)}$. Мы должны доказать, что множество $\left(E^{\prime} f\right)(x) V$ является локальным репером в некоторой точке $c \in \Phi_{C^{(2)}}$ такой, что $c \geqslant \gamma^{(2)}(y)$ для всех $y \in V$. Так как $U^{(2)} \times V$ - локальньй репер в $(A \bowtie B)^{(2)}$, то $\left(E^{\prime} f\right) U^{(2)} V=f\left(U^{(2)} \times V\right)$ - локальный репер в некоторой точке $c \in \Phi_{C^{(2)}}$. Из конструкции отображения $\gamma^{(2)}$ следует, что $c \geqslant \gamma^{(2)}(y)$ для всех $y \in V$. Таким образом, в силу (4.29.1) множество $\left(E^{\prime} f\right)(x) V$ является локальным репером в точке $c$ и $c \geqslant \gamma^{(2)}(y)$ для всех $y \in V$.

Тем самым, мы доказали, что для любого $\alpha \in \Phi_{(A \bowtie B, C)^{(1)}}$ имеет место включение

$$
E_{X}^{\prime}\left(X_{(A \bowtie B, C)^{(1)}}\right)_{\alpha} \subseteq\left(X_{(A,(B, C))^{(1)}}\right)_{E_{\Phi^{(1)}}^{\prime}(\alpha)} .
$$

Чтобы завершить доказательство теоремы, остается показать, что

$$
E_{X}^{\prime}\left(X_{(A \bowtie B, C)^{(2)}}\right)_{\alpha} \subseteq\left(X_{(A,(B, C))^{(2)}}\right)_{E_{\Phi^{(2)}}^{\prime}(\alpha)}
$$

для любого $\alpha \in \Phi_{(A \bowtie B, C)^{(2)}}$. Заметим, что $\operatorname{Mor}(A \bowtie B, C)^{(2)}=\operatorname{Mor}\left(A^{(1)} \times B^{(1)}, C^{(2)}\right)$ и $\operatorname{Mor}(A, \operatorname{Mor}(B, C))^{(2)}=\operatorname{Mor}\left(A^{(1)}, \operatorname{Mor}\left(B^{(1)}, C^{(2)}\right)\right)$. В силу теоремы 3.23 морфизм глобальных действий $E_{X}^{\prime}: \operatorname{Mor}\left(A^{(1)} \times B^{(1)}, C^{(2)}\right) \rightarrow \operatorname{Mor}\left(A^{(1)}, \operatorname{Mor}\left(B^{(1)}, C^{(2)}\right)\right)$ является регулярным и, следовательно, обладает требуемым свойством. 